\title{
Large scale resistivity surveys combining magnetic and magnetotelluric observations
}

- Examples from central Australia -

\author{
Dissertation \\ zur Erlangung des Doktorgrades \\ der Mathematisch-Naturwissenschaftlichen Fakultäten \\ der Georg-August-Universität zu Göttingen
}

vorgelegt von

Ole Hanekop

aus Göttingen

Göttingen 2006 
D7

Referent:

Prof. Dr. Karsten Bahr

Korreferent:

Prof. Dr. Andreas Tilgner

Tag der mündlichen Prüfung: 24.07.2006 


\section{Contents}

1 Introduction $\quad 5$

2 Electromagnetic depth sounding 9

2.1 Basic theory . . . . . . . . . . . . . . . . . 9

2.1.1 The source field . . . . . . . . . . . . . . . . . . . . . .

2.1.2 Maxwell's equations . . . . . . . . . . . . . . . 10

2.1 .3 Induction equation . . . . . . . . . . . . . . . . . . . . . . . . . . . . 12

2.2 Geomagnetic depth sounding . . . . . . . . . . . . . . . . 13

2.2.1 The perturbation tensor .................. 13

2.2 .2 Induction arrows . . . . . . . . . . . . . . 16

2.3 Magnetotellurics . . . . . . . . . . . . . . . . . 17

2.3.1 One dimensional structures . . . . . . . . . . . . . 17

2.3.2 Two dimensional structures . . . . . . . . . . . . . . 19

2.3 .3 Galvanic distortion . . . . . . . . . . . . . . . 20

2.3.4 Dimensionality analysis . . . . . . . . . . . . . 25

2.4 Magnetic distortion . . . . . . . . . . . . . . . . . 26

2.4.1 Decomposition with magnetic distortion . . . . . . . . . 28

2.5 Combination of the MT and GDS methods . . . . . . . . . . . . . . 29

3 Study area and implications from previous surveys 33

3.1 The study area . . . . . . . . . . . . . . . 34

3.2 Crustal and lithospheric thickness . . . . . . . . . . . . . 36

3.3 Seismic tomography results . . . . . . . . . . . . . . . . . . 37

3.4 Gravity surveys . . . . . . . . . . . . . . . . . . . . . . . . . . . . . . . . . . . . . . . . . . .

3.5 Magnetic anomaly data . . . . . . . . . . . . . . . . . . . . 40

3.6 Previous electromagnetic induction results . . . . . . . . . . . . . . . . 41

3.6.1 Other magnetotelluric studies . . . . . . . . . . . . . . 42

4 Field measurements in central Australia 43

4.1 MT results . . . . . . . . . . . . . . . . . . . . . . . . . 44

4.1 .1 Strike angles . . . . . . . . . . . . . . . . . . . . . . . . . . . . . . . . . . 57

4.1 .2 Local induction arrows . . . . . . . . . . . . . . . . . 53

4.2 GDS results . . . . . . . . . . . . . . . . . 54 
5 Combination of magnetic and magnetotelluric transfer functions $\quad \mathbf{5 7}$

5.1 Magnetic to magnetotelluric conversions for noise free 3D model data 58

5.1.1 Conversion of magnetic to magnetotelluric transfer functions . 58

5.1 .2 Multidimensional boundaries . . . . . . . . . . . . . 61

5.2 GDS to MT conversions of the Australian dataset . . . . . . . . . . 62

5.2.1 From tipper to horizontal magnetic transfer functions . . . . . 62

5.2.2 Conversion of magnetic to magnetotelluric transfer functions . 64

5.3 Application to MT data from Germany . . . . . . . . . . . . . . 66

5.4 Guidelines for tipper to GDS/MT conversions . . . . . . . . . . . . 71

6 3D Modeling of the data $\quad 73$

6.1 3D forward modeling . . . . . . . . . . . . . . . . . 73

6.1.1 3D model of the Australian dataset . . . . . . . . . . . . . . 74

6.2 3D inversion . . . . . . . . . . . . . . . . . . . 79

6.3 Structural and geological implications of the models . . . . . . . . . 85

6.3.1 Sediments of the Eromanga Basin . . . . . . . . . . . . . 85

6.3 .2 Mt Isa Block . . . . . . . . . . . . . . . . . . . . . . 86

6.3.3 Carpentaria conductivity anomaly . . . . . . . . . . . . 87

6.3.4 Tasman Line . . . . . . . . . . . . . . . . . . . . . 89

7 Conclusions $\quad 91$

$\begin{array}{ll}\text { A Field sites and data } & 93\end{array}$

A.1 Locations and operating times of the MT sites . . . . . . . . . . . . 93

A.2 Processed MT data . . . . . . . . . . . . . . . . . . . . 94

A.3 GDS results . . . . . . . . . . . . . . . . . . . 94

$\begin{array}{ll}\text { B Programs } & 101\end{array}$

B.1 Programs for display purposes . . . . . . . . . . . . . . . . . 101

B.1.1 emdisp . . . . . . . . . . . . . . . . . 101

B.1.2 d3togmt . . . . . . . . . . . . . . . . . 102

B.2 Data handling programs . . . . . . . . . . . . . . . . . 103

B.2.1 gdsswap . . . . . . . . . . . . . . . . . . . 103

B.2.2 3layer1d . . . . . . . . . . . . . . . 103

B.2.3 multilayer1d . . . . . . . . . . . . . . . . 103

B.2.4 tipper2mt . . . . . . . . . . . . . . . . . 103

B.2.5 Other programs ...................... 104

C GDS to MT transformations for 2D background structures 107

$\begin{array}{ll}\text { D Modeling details } & 109\end{array}$ 


\section{Introduction}

The Australian continent has a long and manifold geological and tectonic history that includes several continental scale rearrangements of different parts of the continent. In the early Proterozoic (2.0-1.8 Ga) three major cratons, the north, west and south Australian cratons, were formed by amalgamation of smaller crustal fragments (Betts et al., 2002). These were assembled between 1.3-1.0 Ga to become part of the early supercontinent Rodinia (Myers et al., 1996; Wingate et al., 2002). Some of these Proterozoic fragments, primarily the Mt Isa Block and the Curnamona province (Broken Hill) host rich ore deposits and are therefore of significant economic importance.

Major parts of south-eastern Australia are of much younger age and were created in the Paleozoic (650-250 Ma) by accretion processes at a westward dipping subduction zone at the eastern continental margin (Betts et al., 2002; Crawford et al., 2003). According to geological investigations no continent-continent collisions occurred in the Palaeozoic. Such collisions are commonly associated with anomalies in seismic wave speeds. Therefore slow wave speeds observed in the mantle below eastern Australia (Debayle \& Kennett, 2000) support the geological investigations. While the seismological data provides some constraints about the possible location of the border between Phanerozoic eastern and Proterozoic central Australia the spatial resolution presently available is insufficient for accurate positioning of this major tectonic boundary. Hill (1951) was the first to define the position of the boundary from geological investigations and introduced the term Tasman Line. As major parts of eastern and central Australia are covered by sedimentary basins, a direct investigation of the basement structures is impossible in many places. Therefore geophysical methods, primarily gravity and aeromagnetic investigations have been used to clarify the exact position of the Tasman Line (Veevers, 1984; Gunn et al., 1997). In places the signatures used to define the Tasman Line are ambiguous, resulting in an ongoing debate about the actual position of the Tasman Line and the correctness of a simple two-dimensional boundary assumption (Direen \& Crawford, 2003; Kennett et al., 2004). In this light additional geophysical investigations are required to get further insight into the crustal structures of central and eastern Australia. Electromagnetic methods are one option to define such structures as different lithospherical compositions and ages are often associated with distinct signatures in subsurface conductivity structures (e.g. Boerner et al. (1999)).

Since the pioneering work of Parkinson (1959) electromagnetic depth sounding methods have been used for structural investigations in Australia. Most campaigns were large scale magnetometer array studies investigating transfer functions between local horizontal and vertical magnetic fields (induction arrows) (Woods \& Lilley, 
1979, 1980; Chamalaun \& Barton, 1993; Chamalaun et al., 1999). These methods are based on electromagnetic induction phenomena in the conducting Earth using time varying magnetic fields generated in the Earth's ionosphere and magnetosphere as source fields. While the most prominent features of Australia's conductivity structures were defined by Australia wide magnetometer array studies (Chamalaun \& Barton, 1993), convincing explanations for the findings are sparse. Partly this is a result of the fact that short period (shorter than some $10000 \mathrm{~s}$ ) geomagnetic depth soundings (GDS) are sensitive to resistivity variations only. Therefore these measurements primarily reveal strong conductivity contrasts rather than smooth variations. Recently additional magnetotelluric (MT) surveys that combine local electric and magnetic field components to investigate regional conductivity structures have been undertaken in Australia (e.g. Simpson (2001); Heinson \& White (2005); Selway et al. (2006)). These measurements allow, at least in principle the resolution of the conductivity of the subsurface. Therefore they are better suited for structural investigations of the substratum. The major limitation of the method is the sensitivity of the electric field to small scale (small compared to the penetration depth) conductivity anomalies. When these structures are spatially unresolved the effect can be treated as a distortion of the electric field and leads to biased resistivity estimates. Distortion effects in MT data can partly be overcome by observations of the phases of the complex impedances. These can be used for investigations of the conductance (product of conductivity and thickness) of structures in the subsurface loosing information about the actual conductivity values.

Like the magnetic field the GDS transfer functions are mostly unaffected by small scale structures when these are negligible for the induction process. Therefore a combination of the GDS and MT methods leads to a better understanding of structures in the subsurface. Becken \& Pedersen (2003) developed an algorithm to convert vertical magnetic transfer functions with good spatial coverage to magnetotelluric transfer functions. While there are several limitations of the conversion algorithm, it allows a valuable connection between magnetic and magnetotelluric transfer functions. Differences between converted and measured transfer functions are often related to the different nature of the induction phenomena and therefore simplify a joint interpretation of the datasets.

In 2003 large scale MT and GDS measurement covering more than $600 \times 900 \mathrm{~km}^{2}$ were performed in central Australia. The measured period range from 8 up to 100000 seconds allows structural investigations in the crust and mantle. The focus in this work lies on crustal structures, including the economically important Mt Isa Block and surrounding basins. The vast extend of the dataset provides good conditions to identify the different crustal units and a possible connection between the previously defined Carpentaria conductivity anomaly (Chamalaun et al., 1999; Lilley et al., 2003) and the Tasman Line.

In Chapter 2 the basic theory of electromagnetic depth soundings is given. Special attention will be paid on distortion phenomena of the electric and magnetic fields. Chapter 3 describes the survey area and reviews the most significant previous investigations. These include geological, gravity, aeromagnetic, seismic and 
electromagnetic results. The results from the 2003 field campaign are presented in Chapter 4. Here first implications for data dimensionality and regional structures will be discussed. These are necessary for the modeling of the data. Chapter 5 introduces GDS to MT conversions for synthetic data from a 3D modeling code, the Australian dataset and a large dataset from Germany using an extended version of Becken \& Pedersen (2003) conversion algorithm that allows input of vertical and horizontal magnetic transfer functions. After the discussion of the conversion results the Chapter concludes with a summary of limitations and benefits of the conversion approach. In Chapter 6 conductivity models to fit the datasets are developed using 3D forward modeling and 3D inversion codes. The forward model includes information from all available datasets, including those of the other geophysical methods and surface geology while the 3D inversion is limited to MT data only. Finally all results are discussed in terms of implications for the crustal architecture of central Australia. Chapter 7 summarizes all results and gives final conclusions. 


\section{Electromagnetic depth sounding}

Electromagnetic depth sounding methods use electric and magnetic fields to resolve conductivity structures within the Earth. In the present work passive methods that use natural electromagnetic variation fields generated in the Earth's ionosphere and magnetosphere are utilized. This chapter describes the theory of electromagnetic depth sounding from first principles with a focus on (i) the geomagnetic depth sounding (GDS) and (ii) the magnetotelluric (MT) method. While the GDS method uses transfer functions between magnetic field components the MT method combines horizontal components of the electric (telluric) and magnetic fields.

\subsection{Basic theory}

\subsubsection{The source field}

The Earth's magnetic field is primarily generated by dynamo processes in the liquid outer core of the Earth. Sensitive measurements however reveal small temporal variations that are of external origin. These geomagnetic variations are the source field used by passive electromagnetic depth sounding techniques. Two major mechanisms lead to the external magnetic fields:

(i) Thermally driven convection of ionized air molecules on the day-side of the Earth: Ultra violet light and soft x-rays from the sun lead to ionization of air molecules in the ionosphere (altitude of $80-120 \mathrm{~km}$ ) on the day-side of the Earth. In conjunction with thermal convection driven by solar heating this leads to large scale electric current systems and therefore the generation of magnetic fields. For low solar activity these are known as solar quiet (Sq) magnetic variations. They are latitude dependent and show seasonal periodicities. The created field amplitudes range from $10-100 \mathrm{nT}$ at a period of about $12 \mathrm{~h}$.

(ii) Interaction of charged particles in the solar wind with the magnetosphere of the Earth: The solar wind is a moving plasma of ionized particles and associated magnetic fields that are expanding outward from the sun. Reaching the Earth the solar wind distorts Earth's magnetosphere that in turn deflects ions along the magnetic field lines. Varying solar wind strength and the deflected ions lead to external magnetic fields measurable at the surface of the Earth. The field amplitudes of the variations depends largely on solar activity and can reach amplitudes of several 100nT at periods of several hours.

Figure 2.1 shows the averaged amplitude spectra of quiet-day geomagnetic activity at mid latitudes. A general trend of increasing amplitudes with longer period can 


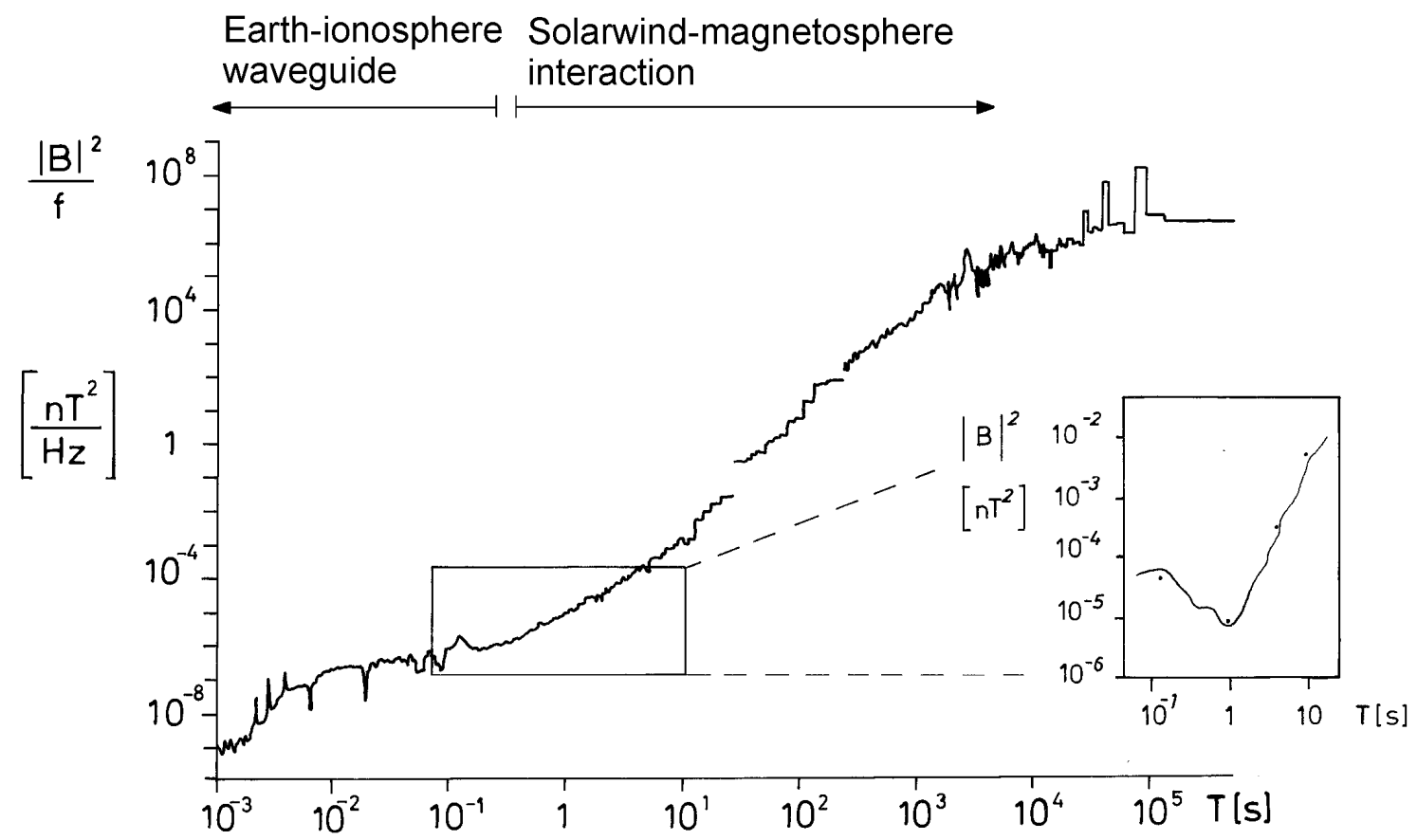

Figure 2.1: Amplitude spectra normalized by the frequency for quiet day geomagnetic variations in mid latitudes (data from central Germany). The inlay shows magnified spectra in the so-called dead band $(10 \mathrm{~Hz}-10 \mathrm{~s})$. From Simpson \& Bahr (2005).

be observed. Peaks exists at the daily variation and its harmonics. Peaks at low frequencies are due to anthropological noise and effects of data filtering to remove these effects (peaks at $50 \mathrm{~Hz}$ (power-lines) and $16.66 \mathrm{~Hz}$ (railway) and their harmonics). The peaks at $8 \mathrm{~Hz}$ and the associated harmonics result from Schumann resonances that are exited by the worldwide thunderstorm activity. For strong geomagnetic storms significantly higher field amplitudes can be observed.

Passive electromagnetic depth sounding methods assume that the source field is horizontally polarized. For moderate solar activity and mid latitudes this plane wave assumption is sensible as the source field is associated with large scale currents that flow at large distances. For high latitudes and around the geomagnetic dip equator however this assumption might no longer be valid. The same is true for very long periods where the curvature of the Earth can no longer be neglected or for strong geomagnetic storms.

A more comprehensive treatment of geomagnetic fields and related phenomena can be found in Campbell (1997).

\subsubsection{Maxwell's equations}

James Clark Maxwell (1873) was the first to publish a universal set of equations that completely describe electromagnetic phenomena. Written in differential form 
Maxwell's equations are:

$$
\begin{gathered}
\nabla \times \mathbf{B}=\mu(\mathbf{j}+\epsilon \dot{\mathbf{E}}) \\
\nabla \times \mathbf{E}=-\dot{\mathbf{B}} \\
\nabla \cdot \mathbf{B}=0 \\
\nabla \cdot \mathbf{E}=\frac{q}{\epsilon},
\end{gathered}
$$

where $\mathbf{E}$ and $\mathbf{B}$ are the electric and magnetic fields, $\mathbf{j}$ the current density, $q$ the charge density, $\epsilon$ the electric permittivity and $\mu$ the permeability. For most electromagnetic depth sounding applications the permeability is close to the free space value $\mu_{0}=$ $4 \pi \cdot 10^{-7} \frac{\mathrm{Vs}}{\mathrm{Am}}$ while the permittivity typically ranges from $\approx 20 \epsilon_{0}$ for most rock types to values of $80 \epsilon_{0}$ for water $\left(\epsilon_{0}=8.85 \cdot 10^{-12} \frac{A s}{V m}\right)$.

As described in the previous section the source field consists of plane waves that are polarized parallel to the surface of the Earth and thus have no vertical source field component. They can be described as a superposition of harmonic waves in a right-handed coordinate system with $x$ in north $y$ in east and $z$ in positive downward direction:

$$
E(t, \mathbf{x}), B(t, \mathbf{x}) \sim e^{i(\omega t+\mathbf{k x})} \cdot e^{|\mathbf{k}| z},
$$

with the angular frequency $\omega$ and wave vector $\mathbf{k}=\left(\begin{array}{l}k_{x} \\ k_{y}\end{array}\right)$.

We use frequencies of less than $100 \mathrm{~Hz}$ in this study. For typical resistivities of the Earth $\left(10^{-3}-10^{5} \Omega m\right)$ this leads to negligible displacement currents $\left(\epsilon_{0} \dot{\mathbf{E}}=i \omega \epsilon_{0} \mathbf{E} \approx\right.$ 0 ) in (2.1). In this so called quasi-static approximation electric field $\mathbf{E}$, current density $\mathbf{j}$ and specific conductivity $\sigma$ are connected by Ohm's law

$$
\mathbf{j}(t, \mathbf{x})=\sigma(\mathbf{x}) \mathbf{E}(t, \mathbf{x})
$$

This leads to the set of Maxwell's equations commonly used in electromagnetic depth sounding:

$$
\begin{array}{r}
\nabla \times \mathbf{B}=\mu_{0} \sigma \mathbf{E} \\
\nabla \times \mathbf{E}=-i \omega \mathbf{B} \\
\nabla \cdot \mathbf{B}=0 \\
\nabla \cdot \mathbf{E}=-\mathbf{E} \nabla(\ln \sigma)
\end{array}
$$

(For transformation of $(2.4)$ to $(2.10)$ see footnote ${ }^{1}$ )

\footnotetext{
${ }^{1}$ From $(2.1)$ and $\nabla(\nabla \times \mathbf{A})=0$ for any $\mathbf{A}$ follows $\nabla(\nabla \times \mathbf{B})=0=\mu_{0} \nabla(\sigma \mathbf{E})=\mu_{0} \nabla \mathbf{j}$. With the vector identity $\nabla(\sigma(\mathbf{x}) \mathbf{E}(\mathbf{x}))=\sigma(\mathbf{x}) \nabla \mathbf{E}(\mathbf{x})+\mathbf{E}(\mathbf{x}) \nabla \sigma(\mathbf{x})$ follows $\nabla(\sigma(\mathbf{x}) \mathbf{E}(\mathbf{x}))=\sigma(\mathbf{x}) \frac{q}{\epsilon_{0}}+$ $\mathbf{E}(\mathbf{x}) \nabla \sigma(\mathbf{x})=0$ and thus $\frac{q}{\epsilon_{0}}=-\mathbf{E} \nabla \ln (\sigma)$
} 


\subsubsection{Induction equation}

In order to find electric and magnetic field solutions for Maxwell's equations the curl of (2.7) and (2.8) has to be calculated

$$
\begin{array}{r}
\nabla \times \nabla \times \mathbf{B}=\nabla(\nabla \cdot \mathbf{B})-\Delta \mathbf{B}=\mu_{0}(\sigma \nabla \times \mathbf{E}-\mathbf{E} \times \nabla \sigma) \\
\Rightarrow \Delta \mathbf{B}=\mu_{0} \sigma \frac{d \mathbf{B}}{d t}+\mu_{0} \mathbf{E} \times \nabla \sigma, \\
\nabla \times \nabla \times \mathbf{E}=\nabla(\nabla \cdot \mathbf{E})-\Delta \mathbf{E}=-i \omega \nabla \times \mathbf{B} \\
\Rightarrow \Delta \mathbf{E}=\mu_{0} \sigma \frac{d \mathbf{E}}{d t}-\nabla(\mathbf{E} \nabla(\ln (\sigma))) .
\end{array}
$$

For a homogenous Earth $(\nabla \sigma=0)(2.12)$ and (2.11) simplify to diffusion equations

$$
\Delta \mathbf{F}=\mu_{0} \sigma \frac{d \mathbf{F}}{d t} \quad \text { with } \quad \mathbf{F}=\mathbf{E}, \mathbf{B} .
$$

For a horizontally incident source field as in (2.5) these have the following solution within the conductive earth (Pedersen, 1989):

$$
\mathbf{F}(t, \mathbf{x})=\mathbf{F}_{1} e^{i(\omega t+\mathbf{k x})} \cdot e^{-|\mathbf{k}| z}+\mathbf{F}_{2} e^{i(\omega t+\mathbf{k x})} \cdot e^{|\mathbf{k}| z}
$$

where position $\mathbf{x}=\left(\begin{array}{l}x \\ y\end{array}\right)$ and wave vector $\mathbf{k}$ are the horizontal parts and $|\mathbf{k}| \equiv$ $k=\sqrt{i \mu_{0} \sigma \omega}$. As the energy sources are outside the Earth the term with increasing field amplitudes with depth is unphysical. Therefore $\mathbf{F}_{2}=0$ and (2.14) simplifies to an attenuated electromagnetic wave. The complex wave number $k$ can be separated in real an imaginary parts

$$
k=\sqrt{\mu_{0} i \omega \sigma} \overbrace{=}^{\sqrt{i}=\frac{i+1}{\sqrt{2}}} \frac{1+i}{\sqrt{2}} \sqrt{\mu_{0} \omega \sigma}=\sqrt{\frac{\mu_{0} \omega \sigma}{2}}+i \sqrt{\frac{\mu_{0} \omega \sigma}{2}} .
$$

While the imaginary part of $k$ describes an oscillation with depth the real part governs the attenuation. The skin depth $p$ is defined as the depth where the original field is reduced to a fraction of $1 / e$ and is given by

$$
p=\frac{1}{\operatorname{Re}[k]}=\sqrt{\frac{2}{\mu_{0} \sigma \omega}} .
$$

For a homogenous earth $(\sigma=$ const $)$ the skin depth represents the depth range of electromagnetic depth sounding methods. Strictly this is no longer true when conductivity gradients are present. In that case equations $(2.12+2.11)$ rather than the simple diffusion equation (2.13) have to be solved. Nevertheless the skin depth is still used as rule of thump for the depth range assuming some average conductivity of the subsurface. As the skin depth is frequency dependent it is convenient to handle the electromagnetic fields in the frequency domain. Therefore the Fourier transform is used to transform the time series into the frequency domain. Note 
that if not indicated otherwise from now on the frequency dependent electromagnetic fields will be addressed.

So far the conductivity $\sigma$ was treated as scalar. Laboratory measurements on rock samples (Kohlstedt \& Mackwell, 1998) and field campaigns (Simpson, 2001; Bahr \& Simpson, 2002; Leibecker et al., 2002; Gatzemeier \& Moorkamp, 2005; Heise \& Pous, 2003) however showed that anisotropic structures exist. In that case the conductivity has to be treated as a tensor rather than a scalar

$$
\underline{\underline{\sigma}}=\left(\begin{array}{ccc}
\sigma_{x x} & \sigma_{x y} & \sigma_{x z} \\
\sigma_{y x} & \sigma_{y y} & \sigma_{y z} \\
\sigma_{z x} & \sigma_{z y} & \sigma_{z z}
\end{array}\right) .
$$

Yin \& Maurer (2001) give a comprehensive treatment of layered structures with arbitrary anisotropy. As electromagnetic methods only provide volume soundings of the conductivity distribution in the subsurface, it is usually not possible to distinguish between microscopic anisotropy in terms of (2.17) and macroscopic variations in subsurface resistivity that are smaller than the skin depth (2.16). Therefore both kinds of anisotropic structures can be modeled by small scale variations in a model with isotropic conductivities in an over-parameterized grid (Moorkamp \& Gatzemeier, 2006; Gatzemeier \& Moorkamp, 2005; Leibecker et al., 2002).

\subsection{Geomagnetic depth sounding}

Geomagnetic depth sounding (GDS) methods use transfer functions between components of the magnetic field to investigate conductive structures within the Earth. The magnetic field measured above a layered structure (called 1D structure in the following) does not depend on the conductivity as long as the curvature of the Earth can be neglected (thus periods up to some $10000 \mathrm{~s}$ in mid-latitudes). For such structures the induced part equals the source field regardless of the conductivity of the subsurface. Therefore it is not possible to extract subsurface conductivity values from magnetic measurements alone. Lateral conductivity contrasts however distort the local magnetic field and produce distinct anomalies in the magnetic field components (Schmucker \& Weidelt, 1975).

\subsubsection{The perturbation tensor}

Schmucker (1970) proposed to separate the magnetic field components in normal and anormalous parts $\mathbf{B}=\mathbf{B}_{n}+\mathbf{B}_{a}$. This is related to the idea of a layered conductivity background associated with the normal field and conductivity anomalies that produce the anormalous parts

$$
\sigma=\sigma_{n}(z)+\sigma_{a}(x, y, z) .
$$

Thus the normal field can be measured in the absence of or far away from anomalous conductivity structures. This lead Schmucker (1970) to the definition of the 
perturbation tensor $\underline{\underline{W}}$

$$
\left(\begin{array}{c}
B_{x}^{a} \\
B_{y}^{a} \\
B_{z}^{a}
\end{array}\right)=\underline{\underline{W}}\left(\begin{array}{c}
B_{x}^{n} \\
B_{y}^{n}
\end{array}\right)=\left(\begin{array}{cc}
h_{H} & h_{D} \\
d_{H} & d_{D} \\
z_{H} & z_{D}
\end{array}\right)\left(\begin{array}{c}
B_{x}^{n} \\
B_{y}^{n}
\end{array}\right) .
$$

Note that as the source field is horizontally polarized there is no normal vertical component for layered conductivity structures. In practice the normal field is usually defined by a normal site above a one dimensional conductivity structure and far from lateral conductivity contrasts. Then the anomalous magnetic field is calculated as the difference between the local and reference fields and the perturbation tensor is defined as

$$
\mathbf{B}^{a}=\mathbf{B}^{l}-\mathbf{B}^{r e f}=\underline{\underline{W}}\left(\begin{array}{c}
B_{x}^{r e f} \\
B_{y}^{r e f}
\end{array}\right) .
$$

Figure 2.2 shows a planar view of the perturbation tensor elements for a simple model of a conductive block $(0.1 \mathrm{~S} / \mathrm{m})$ within a $0.001 \mathrm{~S} / \mathrm{m}$ halfspace and a period of 8 seconds. The GDS transfer functions were calculated using the 3D forward modeling code of Mackie et al. (1994); Mackie \& Booker (1999). As the modeled block is elongated in eastward direction the perturbation tensor elements that were calculated for a magnetic field in northern $\left(B_{x}\right)$ direction $\left(h_{H}, d_{H}, z_{H}\right)$ show the strongest anomalies. This can be understood by looking at the currents induced in the subsurface. Figure $2.2 \mathrm{~b}$ shows a sketch of the current distribution induced by magnetic fields in northern and eastern direction. For a uniform model the current distribution would be equal in both polarizations. The conductive block however channels currents into the block leading to increased current densities within the block and decreased current densities outside the block. As the currents in the subsurface produce secondary magnetic fields in direction perpendicular to the current flow magnetic anomalies can be observed at the subsurface. These are represented by the perturbation tensor elements. Positive values indicate increased magnetic fields relative to the normal field while negative values represent decreased magnetic field values. The following observations can be made:

- for areas far from conductivity anomalies all perturbation tensor elements vanish

- above the pseudo $2 \mathrm{D}$ structure in the middle of the block only the $h_{H}$ element is non-zero with a maximum in the middle of the block. The vertical $z_{H}$ component changes sign with extrema at the borders of the anomaly.

- $d_{H}$ and $h_{D}$ are negligible except at the corners of the block where the currents in Figure 2.2b are bended. Currents that are not perpendicular to the magnetic source field lead to $d_{H}$ and $h_{D}$ anomalies.

- the vertical magnetic field anomalies are highest outside the anomaly and remain at higher values than the horizontal transfer functions. 
a)

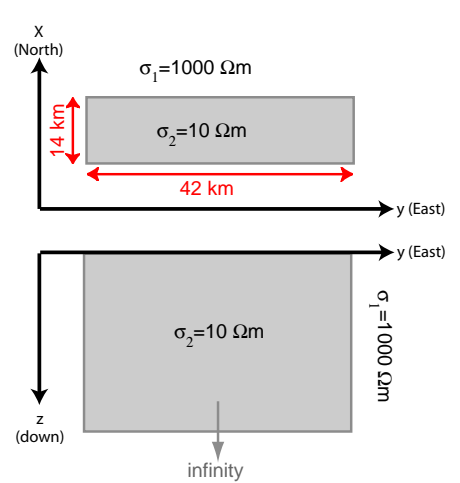

b)

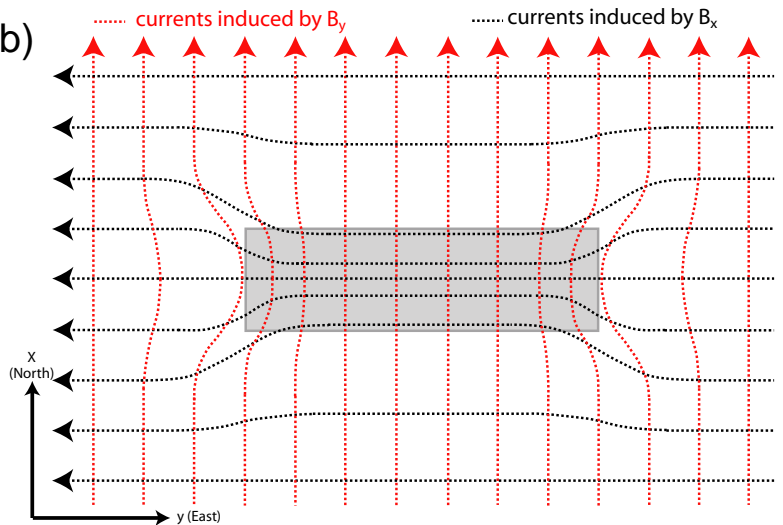

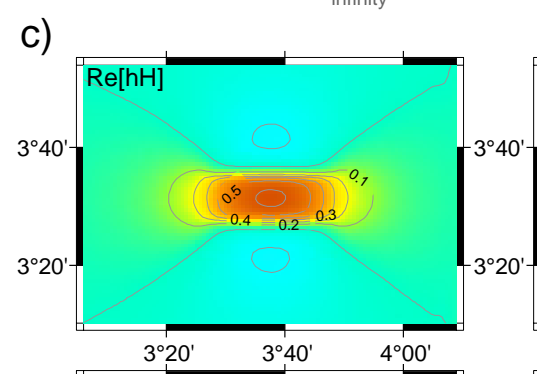
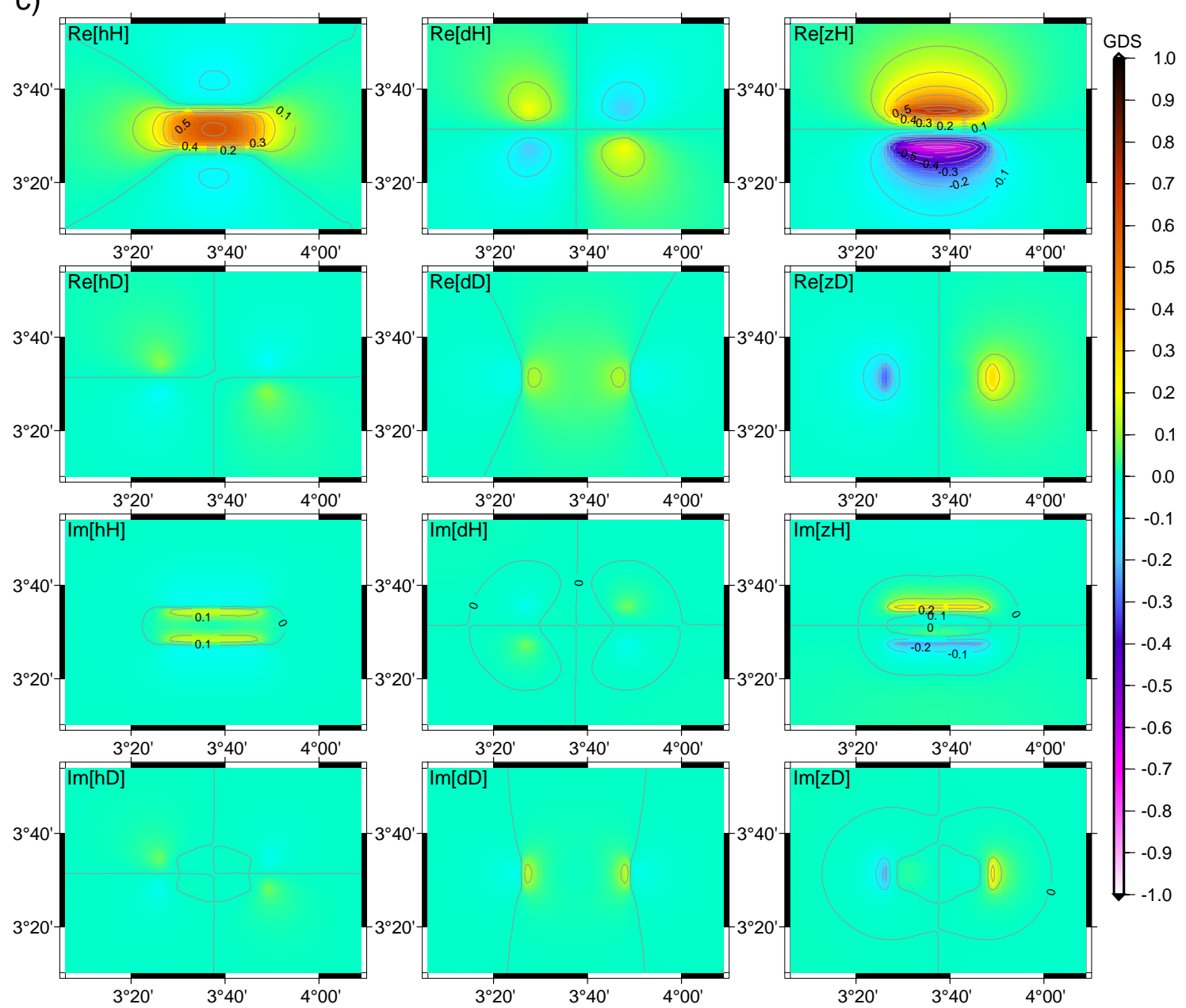

Figure 2.2: (a) Simple model of a conductive block with $0.1 \mathrm{~S} / \mathrm{m}$ in a $0.001 \mathrm{~S} / \mathrm{m}$ halfspace that extends infinitely with depth. (b) Sketch of horizontal current distribution induced by magnetic fields in $\mathrm{x}$ (north) and $\mathrm{y}$ (east) direction. (c) Real and imaginary parts of the perturbation tensor elements for the model in (a) and a period of 8 seconds. The reference station has been chosen far from the anomaly. 
As the perturbation tensor elements depend on the coordinate system they were measured in, these observations are only correct when the anomalous structure strikes in one of the polarization directions. If that is not the case and the structure is purely 2D, the perturbation tensor elements can be rotated by an angle $\alpha$ to the strike coordinate system where $d_{D}$ or $h_{H}$ are maximized:

$$
\left(\begin{array}{cc}
h_{H} & h_{D} \\
d_{H} & d_{D}
\end{array}\right)=\underline{\underline{R}}\left(\begin{array}{cc}
0 & 0 \\
0 & d_{D}^{\prime}
\end{array}\right) \underline{\underline{R}}^{-1} \text { with } \underline{\underline{R}}=\left(\begin{array}{cc}
\cos (\alpha) & \sin (\alpha) \\
-\sin (\alpha) & \cos (\alpha)
\end{array}\right) .
$$

Fluche (1983) developed analytical solutions for rotation angles that minimize the off diagonal elements $d_{H}$ and $h_{D}$ or the ratio of $\left|h_{H}\right| /\left|d_{D}\right|$. Other possible rotation angles could be achieved by a joint minimization of $h_{H}, h_{D}$ and $d_{H}$. In order to avoid effects of the coordinate system, rotational invariants can be used for the interpretation. A sensitivity analysis for several rotational invariants can be found in Leibecker (2000).

A careful selection of the reference site is critical for the success of the method. If conductivity gradients are present close to the reference site this will effect the perturbation tensor elements at all other sites as anomalous field components will be interpreted as normal ones.

One disadvantage of the GDS method is, that in principal the reference site has to be operated for the whole duration of the measurement campaign. It is quite difficult to choose the reference site before the conductivity structure in the ground is known. In practice this problem can be overcome by a change of the reference station. If $\underline{W}^{A, B}$ is the perturbation tensor of a station A with respect to station $\mathrm{B}$ and $\underline{W}^{B, C}$ the tensor for station $\mathrm{B}$ with respect to station $\mathrm{C}$. Than the perturbation tensor for site A with respect to site $\mathrm{C}$ can be found following (2.20) as

$$
\mathbf{B}_{A}-\mathbf{B}_{C}=\overbrace{\left(\underline{\underline{W}}^{A, B} \underline{\underline{W}}^{B, C}+\underline{\underline{W}}^{A, B}+\underline{\underline{W}}^{B, C}\right)}^{\underline{\underline{W}}^{A, C}} \mathbf{B}^{C} .
$$

This can not only be used to change the reference site within a single campaign, but also to connect two separate arrays with at least one common site (Soyer, 2002). Explicit expressions for $\underline{W}^{A, C}$ and the formulas to swap between reference and field sites can be found in Leibecker (2000). These are implemented in the program $g d s s$ wap (see Appendix B.2.1). Note that the error levels of the GDS transfer functions regarding a new reference station highly depend on the quality of the inter-station transfer function between the old and the new reference site.

\subsubsection{Induction arrows}

In the previous section the effect of conductivity gradients on the measured magnetic field has been investigated. In particular the vertical transfer functions $z_{H}$ and $z_{D}$ showed strong amplitudes away from the conductivity gradient (Figure 2.2c). This lead Parkinson (1959) and Wiese (1962) to an arrow representation of these transfer 
functions, the induction vector I

$$
\mathbf{I}=\left(\begin{array}{c}
z_{H} \\
z_{D}
\end{array}\right)
$$

In this definition the induction vectors point away from conductive structures (Wiese (1962) convention), while those defined by Parkinson (1959) point towards the conductor. This can be seen in the change in sign of the $z_{H}$ component in Figure 2.2. The length of the induction vectors are indicators for the amplitudes of the anomalous fields and thus the conductivity gradients. As the transfer functions $z_{H}$ and $z_{D}$ are complex valued, induction arrows of the real and imaginary parts of the transfer functions have to be considered.

In order to investigate conductivity structures from magnetic field observation at single sites it is possible to introduce local induction arrows. These are calculated from the transfer functions $a$ and $b$ between local horizontal and magnetic field components (Schmucker \& Weidelt, 1975)

$$
B_{z}^{l}=a B_{x}^{l}+b B_{y}^{l} \Rightarrow \mathbf{I}^{l}=\left(\begin{array}{c}
a \\
b
\end{array}\right)
$$

For short periods and a reference site above a layered structure normal (2.23) and local (2.24) induction arrows are equal. For very long periods however the curvature of the Earth can no longer be neglected and normal vertical fields are present even for radial dependent conductivity structures.

The other perturbation tensor elements can be plotted in an arrow representation as well. The in that way derived perturbation arrows ${ }^{2}$ rotated by $90^{\circ}$ clockwise represent the anomalous currents induced by one polarization of the normal field.

\subsection{Magnetotellurics}

The Magnetotelluric (MT) method uses transfer functions between horizontal components of the electric and magnetic field on the basis of horizontally polarized source fields. For simple structures these impedances can directly be converted to subsurface conductivity.

\subsubsection{One dimensional structures}

For homogenous conductivity structures the induction equations $(2.12+2.11)$ simplified to diffusion equations with (2.14) as solution. In that case Maxwell's equations

\footnotetext{
${ }^{2}$ Perturbation arrows for each normal field polarization can be defined: $\mathbf{p}=h_{H} \mathbf{e}_{x}+d_{H} \mathbf{e}_{\mathbf{y}}$ and $\mathbf{q}=h_{H} \mathbf{e}_{x}+d_{H} \mathbf{e}_{y}$ with normal vectors $\mathbf{e}_{x}$ and $\mathbf{e}_{y}$. See Schmucker \& Weidelt (1975) for more details.
} 
(2.7) and (2.8) can be evaluated to

$$
\begin{gathered}
E_{x}(\omega)=\frac{k}{\mu_{0} \sigma} B_{y}(\omega), \\
E_{y}(\omega)=-\frac{k}{\mu_{0} \sigma} B_{x}(\omega) .
\end{gathered}
$$

This leads to the definition of the MT impedance

$$
Z(\omega):=\frac{E_{x}}{B_{y}}=\frac{k}{\mu_{0} \sigma} \overbrace{=}^{(2.15)} \frac{i \omega}{k} .
$$

From this definition and (2.25+2.14) Cagnard (1953) derived the apparent resistivity $(\rho=1 / \sigma)$

$$
\rho_{a}(\omega)=\frac{\mu_{0}}{\omega}|Z(\omega)|^{2}
$$

and the phase of the complex impedance

$$
\phi(\omega)=\arctan \left(\frac{\operatorname{Im}(Z(\omega))}{\operatorname{Re}(Z(\omega))}\right) .
$$

Similarly (2.26) leads to the same expression with opposite sign. Introducing the impedance tensor $\underline{\underline{Z}}$

$$
\left(\begin{array}{l}
E_{x} \\
E_{y}
\end{array}\right)=\left(\begin{array}{cc}
0 & Z \\
-Z & 0
\end{array}\right)\left(\begin{array}{l}
B_{x} \\
B_{y}
\end{array}\right) \equiv \underline{\underline{Z}}_{1 D}\left(\begin{array}{l}
B_{x} \\
B_{y}
\end{array}\right)
$$

both results can be combined to one equation.

Note that in some literature a different definition of the MT impedance $(Z=$ $\left.E / H=\mu_{0} E / B\right)$ is used. This leads to different expressions for apparent resistivities and phases.

Schmucker \& Weidelt (1975) introduced the equivalent transfer function $C$

$$
C:=\frac{1}{k}=\frac{Z}{i \omega}
$$

that is directly related to the wave number $k(2.15)$ and thus "a scale length for the depth of penetration into the uniform domain" (Schmucker \& Weidelt, 1975). The real and imaginary parts of both transfer functions ( $Z$ and $C$ ) are not independent but connected by a dispersion relation (Weidelt, 1972). This can be used to check real and imaginary part or apparent resistivity and phase for their consistency although approaches that match the dimensionality of the data have to be applied (Yee \& Paulson, 1988; Egbert, 1990; Yee \& Paulson, 1990; Fischer \& Schnegg, 1993).

When the conductivity changes with depth, the resistivity (2.28) for a given period no longer represents the exact resistivity of the subsurface but a mean value over the induction volume. Wait (1953) developed an analytical solution for the MT impedance of layered structures that is described in more detail in Simpson \& Bahr (2005). 


\subsubsection{Two dimensional structures}

Two dimensional (2D) structures are structures where the conductivity changes with depth and in one horizontal direction. The direction perpendicular to the horizontal conductivity gradient is called strike direction. Let the $\mathrm{x}$-axis be the strike direction $(\sigma=\sigma(y, z))$ then Maxwell's equations $(2.7+2.8)$ simplify to

$$
\left(\begin{array}{c}
\frac{d B_{z}}{d y}-\frac{d B y}{d z} \\
\frac{d B x}{d z} \\
-\frac{d B x}{d y}
\end{array}\right)=\mu_{0} \sigma\left(\begin{array}{c}
E_{x} \\
E_{y} \\
E_{z}
\end{array}\right) \text { and }\left(\begin{array}{c}
\frac{d E_{z}}{d y}-\frac{d E y}{d z} \\
\frac{d E x}{d z} \\
-\frac{d E x}{d y}
\end{array}\right)=-i \omega\left(\begin{array}{c}
B_{x} \\
B_{y} \\
B_{z}
\end{array}\right)
$$

This system of 6 equations decouples in two independent sets of 3 equations

$$
\begin{array}{c|c}
\begin{array}{c}
\text { E-polarization } \\
\text { field components } E_{x}, B_{y}, B_{z}
\end{array} & \text { B-polarization } \\
\frac{d B_{z}}{d y}-\frac{d B_{y}}{d z}=\mu_{0} \sigma E_{x} & \text { field components } B_{x}, E_{y}, E_{z} \\
\frac{d E_{x}}{d z}=-i \omega B_{y} & \frac{d E_{z}}{d y}-\frac{d E_{y}}{d z}=-i \omega B_{x} \\
\frac{d E_{x}}{d y}=i \omega B_{z} & \frac{d B_{x}}{d z}=\mu_{0} \sigma E_{y} \\
& \frac{d B_{x}}{d y}=-\mu_{0} \sigma E_{z}
\end{array}
$$

Following the definition of the MT impedance E- and B-polarization lead to different results. These can be combined in the $2 \mathrm{D}$ impedance tensor

$$
\left(\begin{array}{c}
E_{x} \\
E_{y}
\end{array}\right)=\underline{\underline{Z}}_{2 D} \cdot \mathbf{B}=\left(\begin{array}{cc}
0 & Z_{x y} \\
Z_{y x} & 0
\end{array}\right)\left(\begin{array}{l}
B_{x} \\
B_{y}
\end{array}\right)
$$

where the vanishing main diagonal elements indicate the decoupling of the two polarizations.

In general the measuring coordinate system will not coincide with the system of the electromagnetic strike. In measurement coordinates this leads to a measured impedance tensor $\underline{\underline{Z}}$ with four non-zero elements.

$$
\underline{\underline{Z^{\prime}}}=\left(\begin{array}{cc}
Z_{x x}^{\prime} & Z_{x y}^{\prime} \\
Z_{y x}^{\prime} & Z_{y y}^{\prime}
\end{array}\right)=\underline{\underline{R}}\left(\begin{array}{cc}
0 & Z_{x y} \\
Z_{y x} & 0
\end{array}\right) \underline{\underline{R}}^{-1} \text { with } \underline{\underline{R}}=\left(\begin{array}{cc}
\cos \alpha & \sin \alpha \\
-\sin \alpha & \cos \alpha
\end{array}\right)
$$

As measured impedances are not error free Swift (1967) proposed to minimize the main diagonal elements $\left(\left|Z_{x x}\right|+\left|Z_{y y}\right|\right)$ in order to solve for the rotation angle $\alpha$. This procedure leads to the swift angle

$$
\alpha=\frac{1}{4} \arctan \left(\frac{2 \operatorname{Re}\left[\left(Z_{x y}^{\prime}+Z_{y x}^{\prime}\right) *\left(Z_{x x}^{\prime}-Z_{y y}^{\prime}\right)\right]}{\left|Z_{x x}^{\prime}-Z_{y y}^{\prime}\right|^{2}-\left|Z_{x y}^{\prime}+Z_{y x}^{\prime}\right|^{2}}\right) .
$$


The minimization procedure indicates extrema of main diagonal elements. A maximum can be transformed to a minimum by rotation by $45^{\circ}$. Rotation of a $2 \mathrm{D}$ impedance tensor by $90^{\circ}$ leads to a new 2D tensor with exchanged off-diagonal elements, resulting in an ambiguous strike direction. Without assumptions about the conductivity structure or comparison with additional field sites it is not possible to solve this ambiguity.

As a test whether the 2D assumption of (2.35) is valid Swift (1967) introduced the rotational invariant skew

$$
\kappa:=\frac{\left|Z_{x x}+Z_{y y}\right|}{\left|Z_{x y}-Z_{y x}\right|}
$$

For large skew values $(>0.2-0.3)$ the structure cannot be considered $2 \mathrm{D}$ but has to be treated as three dimensional (3D). Small skew values however do not necessarily indicate $1 \mathrm{D}$ or $2 \mathrm{D}$ structures as they are also observed along symmetry axes of $3 \mathrm{D}$ structures.

Figure 2.3 shows the real parts of all and the apparent resistivities and phases of the off-diagonal elements of the impedance tensor for an in E-W direction elongated block (see Figure 2.2). For the modeled period of 8 seconds only the center part of the block can be considered two dimensional. This is reflected in the small real parts of the diagonal impedance tensor elements that assume their maximum values close to the corners of the block. Even though the eastern and western ends of the block cannot be considered 2D in this case, the diagonal elements vanish along the axes of the block. This leads to vanishing skew values (2.36) along the symmetry axes and maximum skew at the corners of the block (see Figure 2.4a). The phase values are close to their half-space value of $45^{\circ}$ far from the block while they are decreased in E-polarization (yx-component above the center of the block) and increased in Bpolarization (xy-component). The apparent resistivities well inside or far outside the block are close to their true values $(10$ and $1000 \Omega m)$. Close to the boundaries of the block however they reach values that are higher and lower than the modeled values. This distortion is due to charge accumulation at the conductivity boundaries and will be treated in the following section.

\subsubsection{Galvanic distortion}

The definition of the apparent resistivity (2.28) was based on the solution of the diffusion equation (2.13) for homogenous conductivity structures. When conductivity gradients exist not the simple diffusion equation but the equations $(2.12+2.11)$ have to be solved. Without assumptions about the conductivity structure there are no analytical solutions for these generalized induction equations. For horizontally polarized source fields horizontal gradients lead to charge accumulations at the boundaries of different conductivity domains and thus to sources of electric fields. Therefore the divergence of the electric field does not vanish. Equation (2.10)

$$
\nabla \mathbf{E}=\frac{q}{\epsilon_{0}}=-\mathbf{E} \nabla(\ln \sigma)
$$



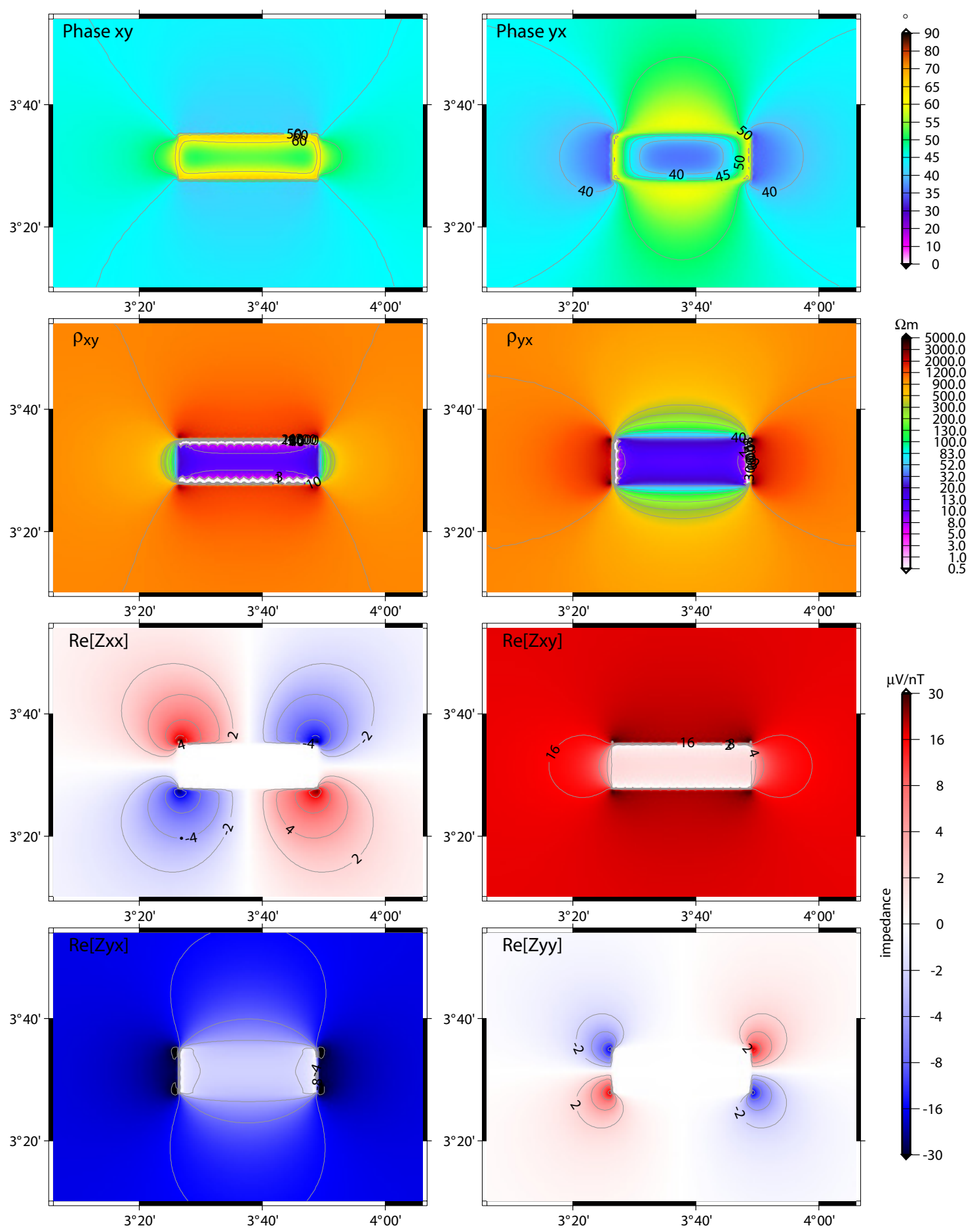

Figure 2.3: MT results for the model of a conductive block within a resistive host medium and a period of 8 seconds. See Figure 2.2 for details. (a) Phases and (b) apparent resistivities of the off-diagonal elements of the MT tensor. (c) shows the real parts of all impedance tensor elements. 
shows that the amount of charge accumulated at the boundary is proportional to the conductivity gradient and the external field strength. This has severe implications for the electric field above conductivity contrasts as the anomalous field created by charge accumulations can be larger than the source field that governs the induction process. For small scale inhomogeneous structures in a homogenous host medium the problem can be treated as scattering of the electromagnetic waves at conductivity inhomogeneities (Groom \& Bahr, 1992; Habashy \& Groom, 1993). For periods with skin depth (2.16) much larger than the extension of an inhomogeneous body the inductive effect of the anomaly is negligible. Therefore only the charge accumulations at the boundaries of the body influence the electromagnetic fields. In the quasi-static approximation localized charges have no effect on the magnetic field. As long as the wavelength of the source field is large compared to the size of the distorting body the effect is frequency independent. Therefore the effect on the MT impedance tensor is called static shift. Mathematically static shift can be described as multiplication of the impedance tensor with a real valued galvanic distortion matrix $\underline{\underline{D}}$ (Larsen, 1977)

$$
\left(\begin{array}{c}
E_{x} \\
E_{y}
\end{array}\right)=\left(\begin{array}{ll}
d_{11} & d_{12} \\
d_{21} & d_{22}
\end{array}\right)\left(\begin{array}{ll}
Z_{x x} & Z_{x y} \\
Z_{y x} & Z_{y y}
\end{array}\right)\left(\begin{array}{l}
B_{x} \\
B_{y}
\end{array}\right)
$$

In general distortion will lead to a fully occupied impedance tensor even for regional 1D or 2D structures in the strike direction. Therefore Swift's minimization procedure results in misleading results that depend on the nature of the distortion. Two different approaches have been used in order to resolve regional 2D structures when distortion is present, an analytical approach by Bahr (1988) and least square fits of the distortion tensor elements (Groom \& Bailey, 1989; Zhang et al., 1987; Smith, 1995).

\section{Phase sensitive decomposition}

The impedance tensor of a regional 2D structure in with purely galvanic distortion and in the strike direction follows from (2.37) as

$$
\underline{\underline{Z}}_{2 D}^{\text {dis }}=\underline{\underline{D}} \cdot \underline{\underline{Z}}_{2 D}=\left(\begin{array}{ll}
d_{11} & d_{12} \\
d_{21} & d_{22}
\end{array}\right)\left(\begin{array}{cc}
0 & Z_{x y} \\
Z_{y x} & 0
\end{array}\right)=\left(\begin{array}{cc}
d_{12} Z_{y x} & d_{11} Z_{x y} \\
d_{22} Z_{y x} & d_{21} Z_{x y}
\end{array}\right) .
$$

In an arbitrary coordinate system this leads to

$$
\underline{\underline{Z}}_{2 D}^{d i s}=\underline{\underline{R}}^{-1} \cdot \underline{\underline{Z}}^{\prime} \cdot \underline{\underline{R}}
$$

As the distortion tensor elements $d_{i j}$ are real valued (only the amplitude of the electric field is influenced by localized currents) the phases in each row of the distorted 2D impedance tensor $\underline{\underline{Z}}_{2 D}^{\text {dis }}$ are equal. This is equivalent to the condition that $\frac{\operatorname{Re}\left[\left(Z_{2 D}^{d i s}\right)_{x x}\right]}{\operatorname{Re}\left[\left(Z_{2 D}^{\text {dis }}\right)_{y x}\right]}=\frac{\operatorname{Im}\left[\left(Z_{2 D}^{d i s}\right)_{x x}\right]}{\operatorname{Im}\left[\left(Z_{2 D}^{d i s}\right)_{y x}\right]}$. Solving for the rotation angle $\alpha$, using this condition and 
the expanded tensor elements in (2.39) leads to the phase sensitive rotation angle (Bahr, 1988)

$$
\alpha=2 \arctan \left(\frac{\left[S_{1}, S_{2}\right]-\left[D_{1}, D_{2}\right]}{\left[S_{1}, D_{1}\right]+\left[S_{2}, D_{2}\right]}\right)
$$

where $S_{1}=Z_{x x}+Z_{y y}, S_{2}=Z_{x y}+Z_{y x}, D_{1}=Z_{x x}-Z_{y y}, D_{2}=Z_{x y}-Z_{y x}$ and $[a, b]:=\operatorname{Re}[a] \operatorname{Im}[b]-\operatorname{Im}[a] \operatorname{Re}[b]$ is the "commutator".

Similar to Swift's skew Bahr (1988) introduced the phase sensitive skew

$$
\eta=\frac{\sqrt{\left|\left[D_{1}, S_{2}\right]-\left[S_{1}, D_{2}\right]\right|}}{\left|D_{2}\right|} .
$$

The interpretation of the phases sensitive skew is similar to Swift's skew. Large values indicate 3D structures while smaller values do not necessarily demand $1 \mathrm{D}$ or 2D structures. For departures from pure 2D structures Bahr (1991) introduced a phase difference $\delta$ in the columns of the impedance tensor that leads to more stable strike directions in such a situation.

Figure 2.4a compares the phase sensitive and Swift rotation angles and the respective skews for the model from Figure 2.2. As there is no anomaly other than the elongated block in the model the resulting angles are similar while the skews show different sensitivities to the more 3D parts of the model. The period dependence however is different for both skews. Swift's skew values increase until the maximum distortion is reached and remains at that level unless other distorting structures are present (Figure 2.4b). The phase sensitive Bahr skew however increases until the three-dimensional effects of the structure are maximal and decreases for longer periods where distortion effects of the conductive block become less significant.

\section{Least square fits of the distortion tensor}

A different approach to resolve the strike direction of $2 \mathrm{D}$ structures when galvanic distortion is present uses a least squares fit of the distortion tensor elements. Groom \& Bailey (1989) show that the distortion matrix $\underline{D}$ can be factorized as

$$
\underline{\underline{D}}=g \underline{\underline{T}} \underline{\underline{S}} \underline{\underline{A}}=g \cdot N_{2}\left(\begin{array}{cc}
1 & -t \\
t & 1
\end{array}\right) \cdot N_{1}\left(\begin{array}{ll}
1 & e \\
e & 1
\end{array}\right) \cdot N_{3}\left(\begin{array}{cc}
1+s & 0 \\
0 & 1-s
\end{array}\right)
$$

where $g$ is the scalar gain factor, $\underline{T}$ the twist, $\underline{\underline{S}}$ the shear and $\underline{\underline{A}}$ the anisotropy tensor and $N_{1}=1 / \sqrt{1+e^{2}}, N_{2}=1 / \sqrt{1+t^{2}}$ and $\overline{\bar{N}}_{3}=1 / \sqrt{1+s^{2}}$ normalization factors. This factorization is closely linked to the Pauli spin matrices known from quantummechanics. It can be shown that any rank 2 tensor has such a representation. The factorization separates the distortion matrix in tensors that have a physical meaning in respect to the electric field involved (Groom \& Bailey, 1989). Note that in strike coordinates the gain $g$ and the anisotropy tensor $\underline{\underline{A}}$ cannot be separated from the regional impedances as $g \underline{\underline{A}} \cdot \underline{Z_{2 D}}$ has the same off-diagonal structure as an undistorted 2D tensor (Groom \& Bailey, 1989; Chave \& Smith, 1994; Smith, 1995; Bibby et al., 2005). 

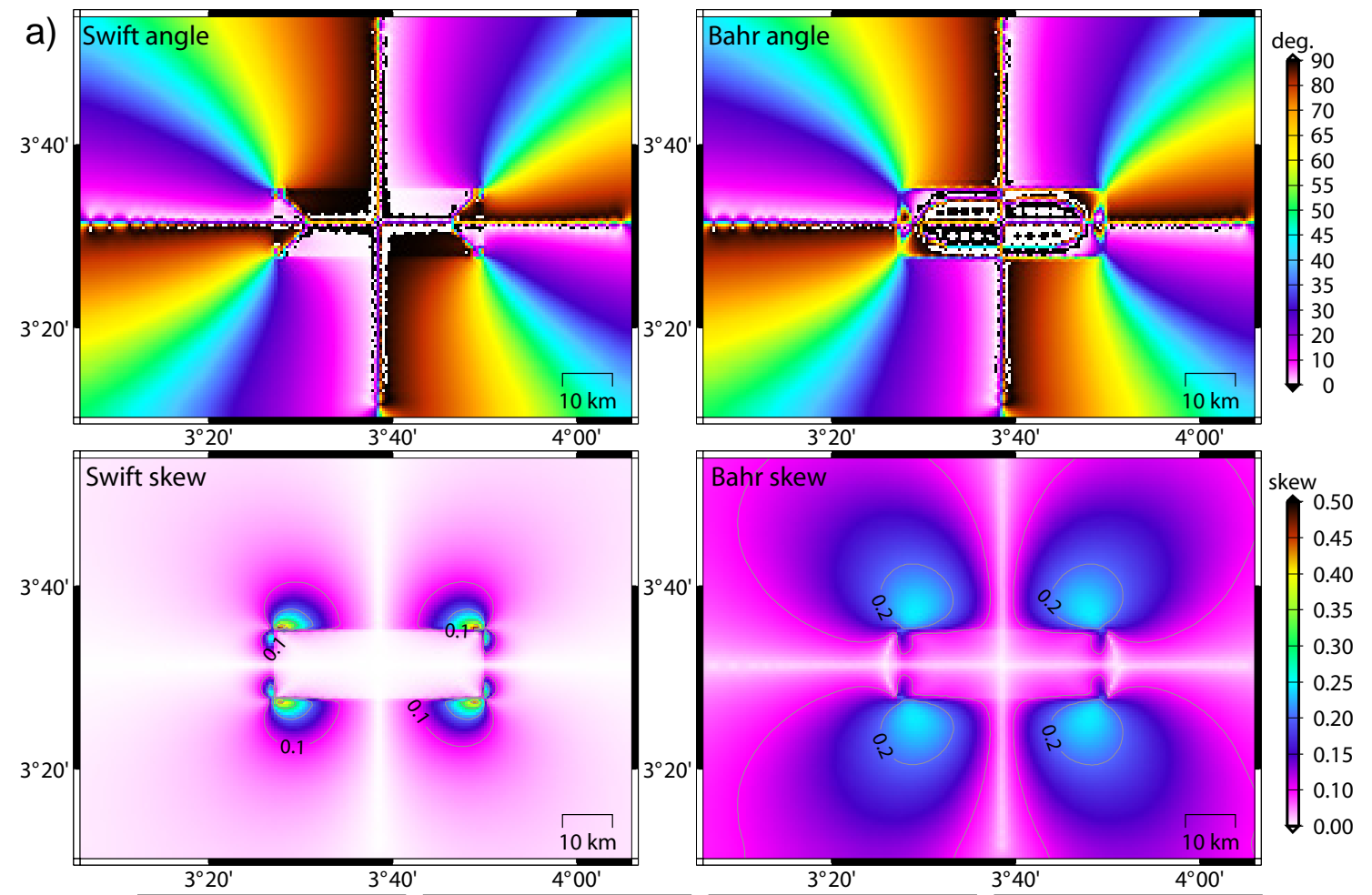

b)
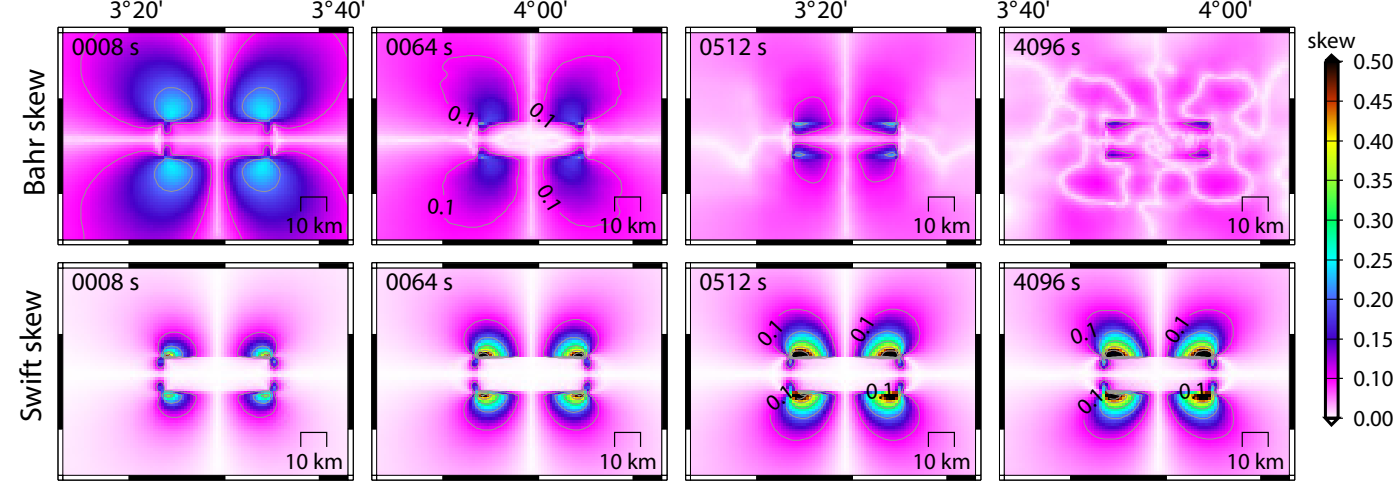

Figure 2.4: (a) Rotational angles according to Bahr (2.40) and Swift (2.35) and the skewnesses (2.41) and (2.36) for the model in Figure 2.2 and a period of 8 seconds. (b) Bahr's and Swifts's skew for the same model and periods from 8 to 4096s.

If the impedance tensor is measured in an arbitrary coordinate system the impedance tensor $\underline{\underline{Z}}^{\prime}$ becomes

$$
\underline{\underline{Z}}^{\prime}=g \underline{\underline{R}} \underline{\underline{T}} \underline{\underline{S}} \underline{\underline{A}} \underline{\underline{Z}}_{2 D} \underline{\underline{R}}^{-1}
$$

The evaluation of this equation leads to a system of 8 real valued equations with 8 real input parameters (the four complex impedance tensor elements) and 7 resolvable unknowns (two complex regional 2D impedances (4 real values), twist $t$, shear $e$ and the rotation angle $\alpha$ ). This system can e.g. be solved by a least square approxi- 
mation $^{3}$. In order to increase the accuracy of the decomposition it is possible to use multiple frequencies and multiple sites to estimate a common strike direction (McNeice \& Jones, 2001). Note that due to the inductive nature of MT data neighboring frequencies are not independent from each other. Therefore adding frequencies to the fit does not increase the degrees of freedom by 8 (one for each real impedance) per frequency.

As the regional impedances were fitted without the determination of site gain and anisotropy tensor they are not necessarily the correct values. Without additional knowledge they can be influenced by a not resolvable static shift. While the normalized misfit can be used to test if the 2D assumption is fulfilled, twist and shear give a hint on the kind of distortion in the data. Bahr (1991) and Balasis et al. (1997) proved the equivalence of the least square and the phase sensitive decomposition in pure $2 \mathrm{D}$ environments by conversion formulas between twist and shear and distortion parameters of the phase sensitive decomposition.

A similar approach to fit the parameters without factorization of the distortion matrix is proposed by Smith (1995) while Becken \& Burkhardt (2004) use an ellipticity criterion to estimate the strike angle. Zhang et al. (1987) describe a solution for local 2D structures as origin of galvanic distortion. Except from some special cases all these decomposition schemes lead to similar strike directions.

\subsubsection{Dimensionality analysis}

One of the main challenges in MT prospecting is the proper identification of the type of structures in the subsurface. In the previous section several methods to characterize possible 1D and 2D structures have been discussed. Due to the complex structure of the Earth, a proper identification of conductivity dimensionality will always be scale dependent. Apart from sites directly above conductivity boundaries, all locations will show different dimensionality for different penetration depth and thus period. This is illustrated in Figure 2.5. While the dimensionality of the structure is obvious in the Figure such a differentiation is difficult for real data. A $2 \mathrm{D}$ anomaly for example has an inductive and distortion response in the impedance tensor. This can be seen in Figure 2.3 where reduced phase values for sites close to the high conductive block indicate the proximity of the block while the apparent resistivities show increased (B-polarization) respectively decreased (E-polarization) values north of the center of the block. Next to the skew values for $2 \mathrm{D}$ structures Bahr (1991) proposed additional rotational invariant measures to distinguish between different distortion types. A different set of invariants and thresholds for the dimensionality analysis have been proposed by Weaver et al. (2000). Recently both methods have been combined to give more stable results (Marti et al., 2005).

As the values of the dimensionality measures depend on the local conductivity structure, it is particularly difficult to define universal threshold values for all ap-

\footnotetext{
${ }^{3}$ As part of this project the Groom \& Bailey (1989) decomposition was included in the program emdisp. For that purpose a modified Levenberg-Marquard algorithm (the lmder subroutine of the minpack project) was used, see Appendix B.1.1
} 

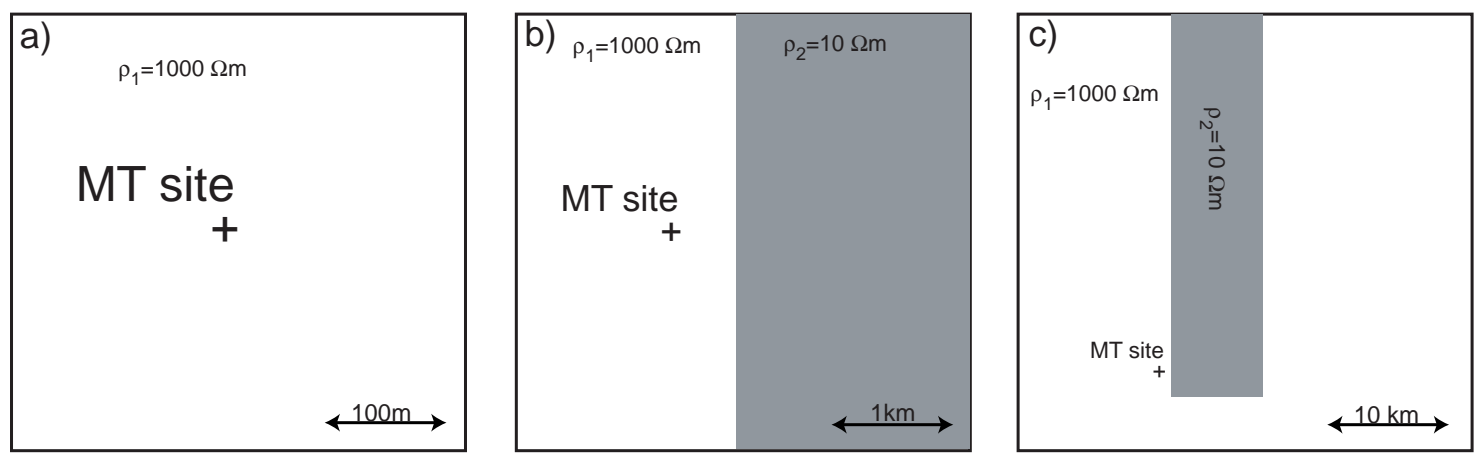

Figure 2.5: Example of dimensionality change with increasing induction space for one station. (a) 1D structure, (b) 2D structure and (c) 3D structure.

proaches. Therefore these methods can only be used as clues but should be followed by careful investigation of other information, in particular data from neighboring sites and possible conductivity contrasts. This can clearly be seen in Figure 2.4a where the skew of Swift's and Bahr's method vanish along symmetry axes of the structure.

\subsection{Magnetic distortion}

In the previous section (2.3.3) the effect of charge accumulations at horizontal conductivity boundaries on the electric field has been discussed. As it is usually not possible to resolve the structure of small scale distorting bodies, it is desirable to completely remove these local effects from MT data. As described earlier this is not possible without additional assumptions, e.g. about the regional structures. The magnetic field at sufficiently long periods is largely unaffected by shallow, small scale structures. In case of elongated structures however electric currents are channeled into conductive bodies. This channeling effects, which produce magnetic anomalies at the surface that are visible in the MT and GDS transfer functions. If the site spacing above the anomaly is too small or the available periods are too long to resolve the structure, the effect can be interpreted as a perturbation rather than a proper conductivity anomaly. As the currents that produce the magnetic anomaly are deflected off the direction defined by the regional structures in an unknown direction the effect can be treated as magnetic distortion. For a regional 1D structure the distortion of the magnetic field is readily described by the perturbation tensor. The galvanic distortion of the anomaly is described by $\mathbf{E}=\underline{\underline{D}} \mathbf{E}^{n}$ where $\mathbf{E}^{n}$ is the normal and $\mathbf{E}$ the measured electric field of the 1D structure. From the definition of the MT impedance tensor $\underline{\underline{Z}}$ follows

$$
\mathbf{E}=\underline{\underline{D}} \mathbf{E}^{n}=\underline{\underline{Z}} \mathbf{B}=\underline{\underline{Z}}\left(\mathbf{B}^{\mathbf{a}}+\mathbf{B}^{\mathbf{n}}\right)
$$


where $\mathbf{B}$ is the measured, $\mathbf{B}^{\mathbf{a}}$ the anomalous and $\mathbf{B}^{\mathbf{n}}$ the normal magnetic field. Multiplication with the row vector $\left(\mathbf{B}^{n}\right)^{-1}=\left(1 / B_{x}^{n}, 1 / B_{y}^{n}\right)$ leads to

$$
\underline{\underline{D}} \mathbf{E}^{n}\left(\mathbf{B}^{n}\right)^{-1}=\underline{\underline{Z}} \mathbf{B}^{\mathbf{a}}\left(\mathbf{B}^{n}\right)^{-1}+\underline{\underline{Z}} \mathbf{B}^{\mathbf{n}}\left(\mathbf{B}^{n}\right)^{-1} \Rightarrow \underline{\underline{D}} \underline{\underline{Z}}^{n}=\underline{\underline{Z}}(\underline{\underline{W}}+\underline{\underline{1}})
$$

where $\underline{\underline{Z}}^{n}$ is the regional 1D impedance tensor $\underline{\underline{W}}$ the horizontal perturbation tensor (2.19) with respect to the $1 \mathrm{D}$ regional structure and $\underline{\underline{1}}$ the identity matrix. If the 1D regional structure is known (e.g. from an undistorted site without anomalies) it is possible to calculate the electric distortion tensor $\underline{D}$. The local perturbation tensor can be interpreted as a distortion of the regional conductivity structure by a magnetic distortion matrix $\underline{\underline{C}}$

$$
\underline{\underline{W}}=\underline{\underline{C}} \underline{\underline{Z}}^{n}
$$

With this definition the measured impedance tensor can be described in terms of the regional impedance and electric and magnetic distortion tensors

$$
\underline{\underline{Z}}=\underline{\underline{D}} \underline{\underline{Z}}^{n}\left(\underline{\underline{C}}_{\underline{Z^{n}}}+\underline{\underline{1}}\right)^{-1}
$$

For periods where galvanic distortion occurs the anomalous magnetic field is in phase with the distorted electric field and thus real valued and largely frequency independent. As the proportion of currents in the surface layer diminishes the effect decreases slowly for very long periods (Chave \& Smith, 1994; Smith, 1997). Agarwal \& Weaver (2000) however argue that for inductive depth magnetic distortion parameters are complex valued as they are related to induced currents in the subsurface. Their numerical modeling study therefore implies that the assumption of real valued magnetic distortion might be wrong or at least not applicable for arbitrary structures.

The vertical magnetic field is affected by magnetic distortion in a similar way as the horizontal field. This can be included in a 2x3 magnetic distortion matrix. From $\mathbf{B}^{a}=\underline{C} \underline{Z}^{n} \mathbf{B}^{n}$ follows (Ritter, 1996; Ritter \& Banks, 1998)

$$
B_{z}^{a}=\left(D_{z x}, D_{z y}\right) \underline{\underline{Z}}^{n} \mathbf{B}^{n}
$$

Comparison with the normalized induction arrows (2.23) leads to

$$
\left(z_{h}, z_{d}\right)=\left(D_{z x}, D_{z y}\right) \underline{\underline{Z}}^{n}
$$

For 1D structures and wavelength that are small compared to the curvature of the Earth the local and normal induction vectors are equal. In more complex situations the solution is more complicated as spatially varying horizontal and vertical anomalous magnetic fields come into play. These mix with the local distorting anomalous field. The local horizontal anomalous field regarding a 1D site that is unaffected by the regional structure becomes

$$
\mathbf{B}^{a}=\mathbf{B}_{l}^{a}+\mathbf{B}_{r}^{a}=\underline{\underline{W}} \mathbf{B}^{n}=\left(\underline{\underline{W_{l}}}+\underline{\underline{W_{r}}}\right) \mathbf{B}^{n} .
$$


In practice a separation of local and regional magnetic distortion effects is difficult. For arrays in the vicinity of a regional $2 \mathrm{D}$ structures a separation is possible if multiple sites are effected by the same local anomaly (Ritter \& Banks, 1998). As the regional field has to be uniform over the sites effected by local distortion this approach is limited to small arrays sufficiently removed from the regional structure.

\subsubsection{Decomposition with magnetic distortion}

Section 2.3.3 dealt with the effects of galvanic distortion on the MT impedance tensor. For 2D structures different approaches to resolve the regional strike direction have been presented. According to equation (2.47) the measured impedance tensor can be influenced by magnetic distortion. This leads to a wrong tensor decomposition when only galvanic distortion is assumed. For a real valued magnetic distortion tensor Chave \& Smith (1994) developed a decomposition on the basis of (2.47) that leads to the following impedance tensor in an arbitrary coordinate system

$$
\underline{\underline{Z}}=\underline{\underline{R}} \underline{\underline{T}} \underline{\underline{S}} \underline{\underline{Z}}_{2 D}\left(\underline{\underline{C}}_{\underline{\underline{Z}}} \underline{\underline{1}}^{+} \underline{\underline{\underline{1}}}^{-1} \underline{\underline{R}}^{-1}\right.
$$

Here the same factorization as in the Groom \& Bailey (1989) decomposition is used $(\underline{\underline{D}}=\underline{\underline{T}} \underline{\underline{S}}$, see Section 2.3.3). Chave \& Smith (1994) showed that only the diagonal elements of the magnetic distortion tensor can be estimated from the measured tensor. The off-diagonal elements are not distinguishable from the regional impedances and can thus be set to unity. This leads to a problem with 8 input parameters (4 complex impedances) and 9 unknowns (twist $t$, shear $e, C_{x x}:=\gamma, C_{y y}:=\epsilon$ the regional strike $\alpha$ and the two complex regional impedances). This system cannot be solved for a single frequency. As the distortion parameters $e, t, \gamma, \epsilon$ and the regional strike are frequency independent more than one frequency can be used to fit the 9 parameters to the data. This can be done in a similar way as the least square fit for multiple frequency Groom \& Bailey (1989) decomposition. When the misfit is investigated it should be noted that due to the inductive nature of MT data the degrees of freedom do not increase by 8 (one for each real/imaginary part of the measured impedances) per frequency. Smith (1997) developed a similar decomposition with a slightly easier iterative approach to fit the parameters ${ }^{4}$. Both decompositions can be extended with local magnetic transfer functions leading to a slightly better posed problem (4 additional input parameters and only $2\left(C_{z x}, C_{z y}\right)$ additional magnetic distortion parameters).

In case of complex valued distortion parameters all these decompositions do not lead to better results than the galvanic distortion only decompositions. In that situation the magnetic distortion parameters are inextricably mixed with the regional impedances and no decomposition scheme will be successful.

\footnotetext{
${ }^{4}$ Within the scope of this study both, Chave \& Smith (1994) and Smith (1997) decompositions have been implemented into the program emdisp, see Appendix B.1.1
} 


\subsection{Combination of the MT and GDS methods}

Maxwell's equations (2.7-2.10) combine electric and magnetic fields. With some limitations it is therefore possible to convert vertical magnetic to GDS or MT transfer functions (Becken \& Pedersen, 2003).

The vertical and horizontal components of the magnetic field are combined by the Hilbert transformation. At the surface $\left(z=z_{0}\right)$ above an infinitely extended plane the Hilbert transformation in the wave number domain is (Nelson, 1986; Pedersen, 1989)

$$
\widetilde{\mathbf{B}}_{h}\left(\mathbf{k}, z_{0}\right)=\frac{i \mathbf{k}}{k} \widetilde{B}_{z}\left(\mathbf{k}, z_{0}\right)
$$

where the tilde indicates field values in the wave number domain, $\mathbf{k}=\left(\begin{array}{c}k_{x} \\ k_{y}\end{array}\right)$ is the horizontal wave number, and $k=|\mathbf{k}|$. This solution is closely related to the horizontal and vertical gradients of the magnetic field in (2.14). In the space domain the Hilbert transformation can be described as integral equation (Nelson, 1986; Gharibi \& Pedersen, 1999). When array data is available (2.52) can be used to calculate the local horizontal GDS anomalies from the local vertical magnetic transfer functions (induction arrows). Remembering that only anomalous (non 1D) structures lead to vertical magnetic fields the horizontal magnetic field can be described as (Becken \& Pedersen, 2003)

$$
\widetilde{\mathbf{B}_{h}}(\mathbf{k})=\widetilde{\mathbf{B}_{h}^{n}}+\widetilde{\mathbf{B}_{h}^{a}}(\mathbf{k}) \overbrace{=}^{(2.52)} \widetilde{\mathbf{B}_{h}^{n}}+\frac{i \mathbf{k}}{k} \widetilde{B_{z}}(\mathbf{k}) \overbrace{=}^{(2.24)} \widetilde{\mathbf{B}_{h}^{n}}+\frac{i \mathbf{k}}{k}\left(\widetilde{\mathbf{I}^{T}}(\mathbf{k}) * \widetilde{\mathbf{B}_{h}}(\mathbf{k})\right)
$$

where $*$ denotes the convolution operator. This is the wave number domain representation of a Fredholm integral equation of the second kind that can be solved by successive approximation and Fourier transformations between space and wave number domain. As first approximation the local vertical field can be obtained from the vertical transfer functions (induction arrow) and the normal horizontal field for one of the two perpendicular source field polarizations

$$
B_{z}^{j+1}(\mathbf{r})=\mathbf{I}^{T}(\mathbf{r}) \mathbf{B}_{h}^{j}(\mathbf{r}) .
$$

In a second step the estimated vertical magnetic field is transformed to the wave number domain to approximate the anomalous horizontal field

$$
\widetilde{\mathbf{B}}_{h}^{j+1}(\mathbf{k})=\frac{i \mathbf{k}}{k} \widetilde{B_{z}^{j+1}}(\mathbf{k}) .
$$

Finally the anomalous horizontal field is transformed back to the space domain to get a new estimate of the total horizontal field in the space domain

$$
\mathbf{B}_{h}^{j+1}(\mathbf{r})=\mathbf{B}_{h}^{a, j+1}(\mathbf{r})+\mathbf{B}_{h}^{n} .
$$

Iterations of these three steps are performed until the difference of the vertical component at a given location is less than some $\delta B$. The anomalous horizontal field 
components represent the GDS perturbation tensor elements. Those for a normal field $\mathbf{B}_{n}^{H}=\left(\begin{array}{c}1 \\ 0\end{array}\right) n T$ represent $h_{H}$ and $d_{H}$ and those for the perpendicular normal field $\mathbf{B}_{n}^{D}$ the tensor elements $h_{D}$ and $d_{D}$.

In order to estimate the associated electric fields the nature of magnetic transfer functions has to be analyzed. As the magnetic flux $\mathbf{B}$ and the current density $\mathbf{j}$ are divergence free they can be decomposed into toroidal and poloidal parts (Schmucker \& Weidelt, 1975). Denoting fields that are related to toroidal currents with the subscript $E$ and those related to poloidal currents with subscript $M$ leads to a decomposition for the magnetic and electric fields. The toroidal vectors $\mathbf{j}_{E}$ and $\mathbf{E}_{E}$ together with the poloidal vector $\mathbf{B}_{E}$ define the tangential electric (TE) mode while the toroidal vector $\mathbf{B}_{M}$ and the poloidal vectors $\mathbf{j}_{M}$ and $\mathbf{E}_{M}$ are the tangential magnetic (TM) mode. In magnetotelluric context these terms are often mixed with the E- and B-polarization introduced in Section 2.3.2. While the latter terms are only meaningful for $2 \mathrm{D}$ structures TE and TM mode are directly connected to the electromagnetic fields. In insulating media (e.g. just outside the conductive Earth) $E_{M}$ and $B_{E}$ are potential fields and $\nabla \times \mathbf{E}_{M}=\mathbf{0}$. From (2.9) follows $\mathbf{B}_{M}=\mathbf{0}$. This implies poloidal currents in the Earth cannot produce toroidal magnetic fields at the Earth's surface and that only poloidal magnetic fields exists outside of the Earth (Berdichevsky \& Zhdanov, 1984). Therefore magnetic transfer functions are entirely of TE-mode. As the electric field measured at the surface of the Earth consists of TE and TM parts only the TE mode electric fields can be estimated from magnetic transfer functions. Following Maxwell's equations and noting that toroidal fields have no vertical component (i.e. $\mathbf{E}_{E}^{T}=\left(E_{x}, E_{y}, 0\right)$ ) we get

$$
\nabla \times \mathbf{E}_{E}=-i \omega \mathbf{B}_{E} \quad \Rightarrow \quad\left(\begin{array}{c}
-\frac{\partial E_{y}}{\partial z} \\
\frac{\partial E_{x}}{\partial z} \\
\frac{\partial E_{y}}{\partial x}-\frac{\partial E_{x}}{\partial y}
\end{array}\right)=-i \omega\left(\begin{array}{c}
B_{x} \\
B_{y} \\
B_{z}
\end{array}\right)
$$

For horizontally polarized source fields as in (2.14) the partial derivative $\frac{\partial}{\partial z}$ equals a multiplication by $k=|\mathbf{k}|$. Transformation into the wave number domain and application of the Hilbert transform (2.52) leads to

$$
\left(\begin{array}{c}
-\tilde{E}_{y} \\
\tilde{E}_{x}
\end{array}\right)=\frac{\omega}{k^{2}}\left(\begin{array}{c}
k_{x} \\
k_{y}
\end{array}\right) \tilde{B}_{z}
$$

Division by $k^{2}$ causes numerical instability at $k=0$, the constant component in the wave number domain. Setting $\mathbf{E}(k=0)=0$ can be used to solve the instability. Using different values would simply add a constant to the electric fields at all locations in the space domain.

Using (2.55) in equation (2.58) allows the calculation of the TE mode electric field in the wave number domain that can be back transformed to the space domain. Apart from a constant shift this leads to the local electric field components. If an undistorted MT impedance $\underline{\underline{Z}}$ is known at one point which is sufficiently removed from multidimensional structures this impedance can be used as reference for the 
electric field. As both, electric and magnetic are known the electric field can easily be shifted to the value demanded by the known 1D impedance and the impedances at all other locations can be estimated. The major limitation is the fact that only the TE mode electric field can be estimated. For 2D structures this results in the fact that only the E-polarization MT impedances can be calculated. Such a result is not surprising as the vertical magnetic transfer functions that were used at the beginning vanish in strike direction of a $2 \mathrm{D}$ contrast (the induction arrows point away from the conductor). As the whole system decouples and no vertical component in one polarization exists location independent, constant B-polarization impedance tensor elements are obtained. In order to estimate the effect on the dataset investigated in thesis study the algorithm is implemented in the program tipper2mt (see Appendix B.2.4).

A different application of the Hilbert transformation is to test the consistency of the vertical transfer functions (Marcuello et al., 2005). In a similar way as for the impedance tensor the dispersion relation between real and imaginary part of the transfer function is used to calculate one from the other. If the calculated responses do not match the measured ones the transfer functions are not consistent and should not be interpreted.

In addition the vertical magnetic transfer functions can be used to remove static shift effects from MT data. Utada \& Munekane (2000) developed an algorithm where the distortion tensor elements (Section 2.3.3) at local MT sites can be estimated in a least square approach. Next to the vertical magnetic transfer functions their approach requires knowledge of the spatial derivatives (and therefore good spatial coverage) of the impedance tensor elements. 


\section{Study area and implications from previous surveys}

The Australian continent has a diverse and manifold geological history. The oldest exposed parts of the continent are the Archean Pilbara (major tectonic evolution from 3.65 Ga onwards), Yilgarn $(3.73 \mathrm{Ga}$ ) and Gawler (2.64 Ga) cratons (Figure 3.1, Betts et al. (2002)). Most of western and central Australia was formed by amalgamation processes in the Paleoproterozoic and Proterozoic (2500-1000 Ma). Eastern Australia is dominated by the much younger Tasman fold belt which was formed about 800-600 Ma ago (Crawford et al., 2003). The transition between Proterozoic western and central Australia and the younger eastern part is commonly referred to as Tasman line (Figure 3.1a, Hill (1951); Veevers (1984)). It is frequently brought in context with the break-up of the early supercontinent Rodinia (600-780 Ma, Wingate et al. (2002)). In the succession of this break-up, provinces east of the Tasman line accreted to the continent. In the last 50 years a number of different possible locations for the Tasman line have been postulated on the basis of outcropping basement rocks, magnetization and gravity surveys. Some of these fall into the region studied by this project. If the Tasman line is the boundary between younger and older parts of the continent it should also mark a major boundary for geophysical parameters like density, seismic wave speeds and subsurface resistivity.

Recently there has been some dispute about the idea of a simple Tasman line. Direen \& Crawford (2003) point out that a number of different tectonic events lead to the creation of faults and subsequent gravity and magnetic anomalies interpreted as Tasman line in southern Australia. While for northern Queensland the idea of one continuously connected north-south trending Tasman line might be appropriate it is an oversimplification in south-eastern Australia. As major parts of the Tasman line in central Australia are obscured by sediments geophysical techniques are required to clarify the location of the boundary.

The most important Proterozoic and Archean geological terrains and one possible location of the Tasman line are mapped in Figure 3.1a. Up to $3 \mathrm{~km}$ thick sedimentary basins cover the areas in-between those regions in the survey area of this study, preventing their geological classification (Figure 3.1b). Therefore several geophysical techniques have been used to investigate the continuation of geologically defined structures with depth and to resolve structures below the covering sedimentary basins.

In 2003 electromagnetic field measurements were performed in central Australia. This Chapter focuses on the region investigated by these measurements. First the field campaign and the major geological structures in the area of interest are intro- 
a)

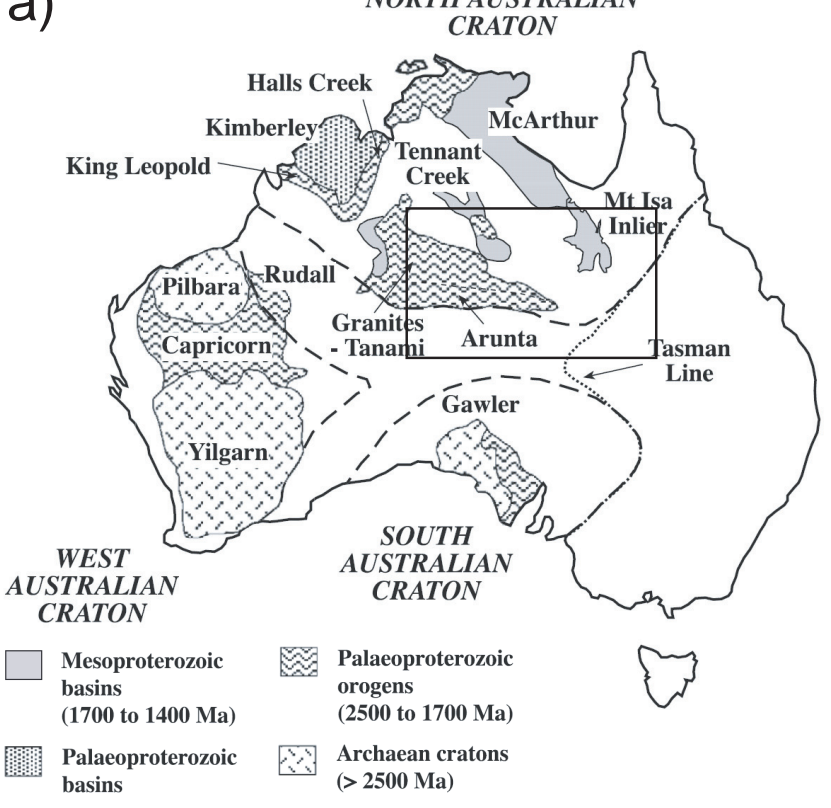

b)

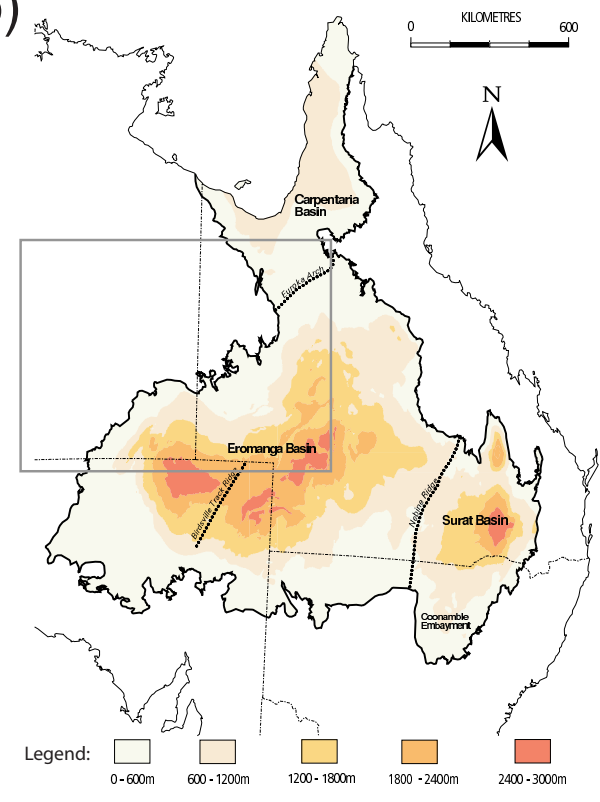

Figure 3.1: (a) Terrane map of the Australian plate indicating the major geological structures (adapted after Bierlein \& Betts (2004)). (b) Sedimentary thicknesses in the Great Artesian Basin (modified from GABCC (1998)). The boxed areas indicate the survey region of this study.

duced. Afterwards implications from previous geophysical investigations, including seismological, aeromagnetic, gravity and electromagnetic measurements and their interpretation in terms of major structural features of the Australian continent and the Tasman line are summarized. In the following Chapters 4 and 6 these results will be compared to the electromagnetic results of this study. While methods that are sensitive to different physical properties of the Earth do not necessarily resolve the same structures a combination is sensible for major tectonic boundaries that are often associated with variations in various parameters.

\subsection{The study area}

The 2003 electromagnetic field campaign scanned an area of more than $600 \times 900$ $\mathrm{km}^{2}$ in terms of electrical conductance of the subsurface, covering a good portion of central Australia. Figure 3.2 shows a topographic map of the study area. The symbols on the map represent the locations of magnetotelluric sites installed during our campaign in mid 2003. Sites shown as red inverted triangles were operated for about 14 h only while those marked by blue stars recorded for 6-60 days. The exact operation times and site locations can be found in Appendix A.1.

Different geological units fall into the surveyed region (Figure 3.1). The most significant are the outcropping Paleoproterozoic Arunta Block in the west and the 


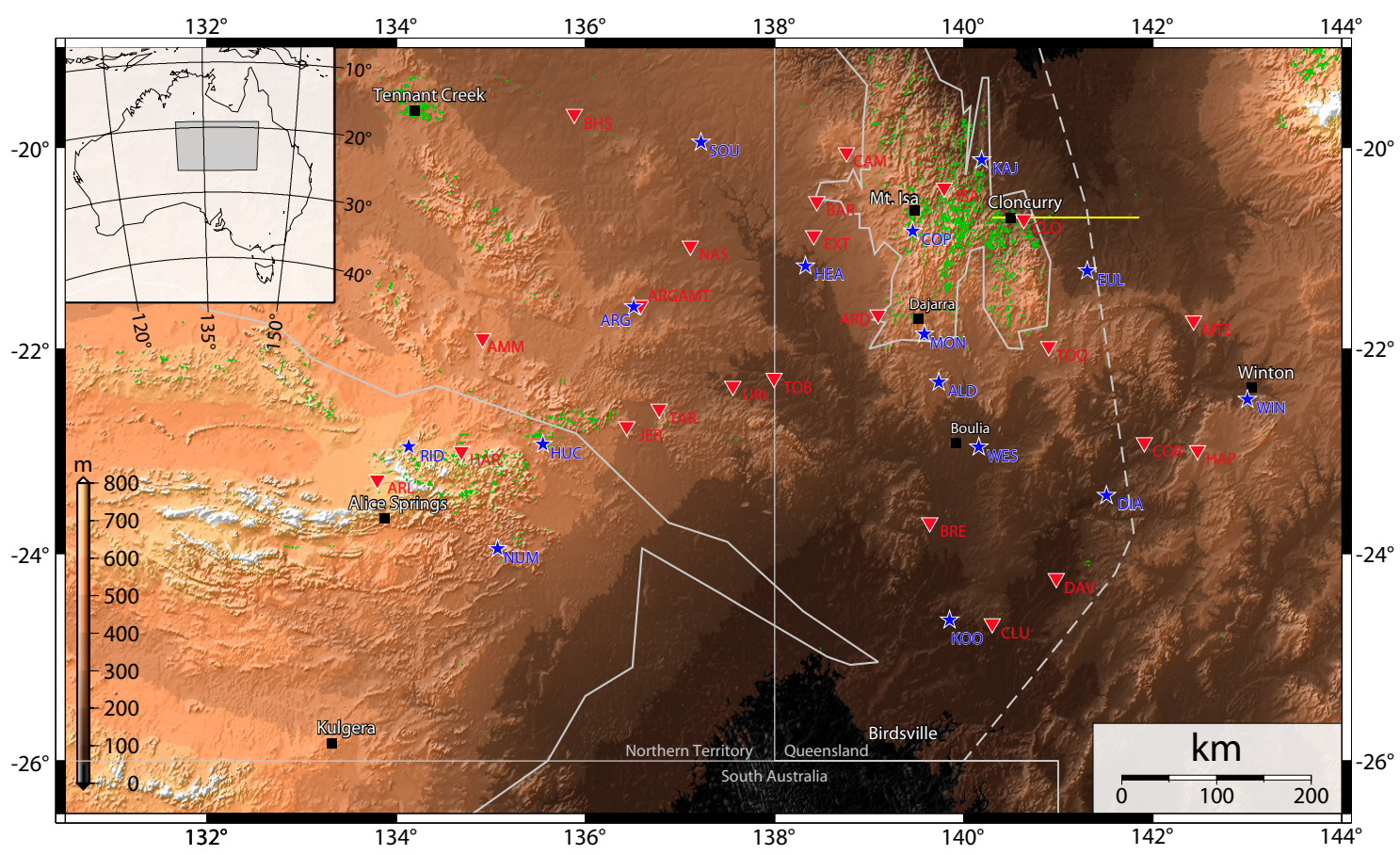

Figure 3.2: Topographic map and general geological structures of the study area. The upper left inclusion shows the location of the area on the Australian continent. Red triangles and blue stars represent MT sites. Blue stars indicate long period sites that have been installed for several days, while red triangles indicate short-period sites with $14 \mathrm{~h}$ of data. Thick grey lines outline the Mt Isa Inlier (top/center) and the Arunta Block (lower/left). The dashed line marks the Carpentaria conductivity anomaly and the thin yellow line follows the MT profile of Lilley et al. (2003) (Figure 3.7). Green dots indicate the location of operating and abandoned mines. Topography data from Petkovic \& Buchanan (2002).

Proterozoic Mt Isa Inlier in the north-east (Betts et al., 2002). The other areas are covered by sedimentary basins notably the late Proterozoic Georgina Basin (north-west) and the Mesozoic Eromanga Basin (south) (Chamalaun et al., 1999).

Several conflicting theories exist for the creation of the Arunta Block. These include accretion processes at the southern boundary on the North Australian craton (Betts et al., 2002) as well as 1.3 to 1.1 Ga activity during rearrangements of the North and South Australian Cratons (Giles et al., 2004). Proterozoic subduction at the southern boundary of the North Australian craton is one mechanism that might explain large scale back-arc extension that lead to the creation of the McArthur Basin and activity in the Mt Isa Block (Betts et al., 2002; Betts, 1999; Giles et al., 2002).

Based on seismic reflection and refraction surveys (MacCready et al., 1998) the central Mt Isa Inlier with it's north-south striking faults was assumed to be the 
boundary between two major blocks of the Australian continent (the North Australian and North Queensland mega elements, Drummond et al. (1998)). Gravity and geochemical measurements however indicate that the basement on either side of the major faults in the Mt Isa Block must have been created in close proximity (Bierlein \& Betts, 2004). A possible boundary between major blocks (which can be assumed from gravity data, Section 3.4) has to be outside of the inlier. The Mt Isa Block is economically important as it hosts some of world's largest $\mathrm{Pb}-\mathrm{Zn}-\mathrm{Ag}-\mathrm{Cu}$ ore bodies (Bierlein \& Betts, 2004). The location of present and abandoned mines is shown as green dots in Figure 3.2 (data from Ewers et al. (2001)).

The Eromanga Basin is an up to $3 \mathrm{~km}$ thick sedimentary basin (Figure 3.1b, Clitheroe et al. (2000a) infer $2 \mathrm{~km}$ from models based on sparsely spaced seismological data). It hosts part of the Great Artesian Basin (Figure 3.1b), central Australia's major subsurface water reservoir. The water is stored in aquifers between impermeable layers of clay and sandstone that were formed at $200 \mathrm{Ma}$ when the area was covered by a shallow sea. While water from the Great Artesian Basin is suitable for drinking, its salinity makes it unsuitable for irrigation in most areas. The oldest groundwater in the Basin has been isotopically dated at close to $2 \mathrm{Ma}$ (in the south-eastern part of the Basin, Habermehl \& Pestov (2002)). As fluids with high mineral content provide an important conduction mechanism in the Earth's crust the Eromanga Basin can be expected to be a prominent structure in terms of subsurface conductivity.

\subsection{Crustal and lithospheric thickness}

Figure 3.3 shows the crustal thickness in terms of a Moho-depth ${ }^{1}$ map of the Australian continent. The data was compiled from seismological receiver function and seismic reflection surveys and is in agreement with the petrologically defined boundary between crust and lithospheric mantle (Clitheroe et al., 2000b). In most areas the moho is defined by a sharp to moderate increase of the seismic wave speeds over a depth range of $1-5 \mathrm{~km}$. Areas with particularly thick crust, e.g. below the Mt Isa Block $(55 \mathrm{~km})$ however show a $15 \mathrm{~km}$ wide transition zone, indicating slow compositional and thermal changes at Moho depths (Simons \& van der Hilst, 2002; Collins et al., 2003). East of about 140 degree east Clitheroe et al. (2000b) found a distinct transition from thick crust below central Australia to thinner crust of about 35-40 km, near Australia's continental average of $38.3 \mathrm{~km}$, (Collins et al., 2003) in eastern Australia (grey line in Figure 3.3). This transition possibly indicates a seismological representation of the Tasman line.

The thickness of the lithosphere varies significantly in central Australia (Simons \& van der Hilst, 2002; Collins et al., 2003; Direen \& Crawford, 2003). Similar to crustal thicknesses the thickest lithosphere can be found in Proterozoic central Australia rather than the Archean cratons. Therefore the lithospheric thickness is

\footnotetext{
${ }^{1}$ The so-called Mohorovicic (Moho) discontinuity defines seismically the crust-mantle boundary. It originates from a change in rock-composition.
} 


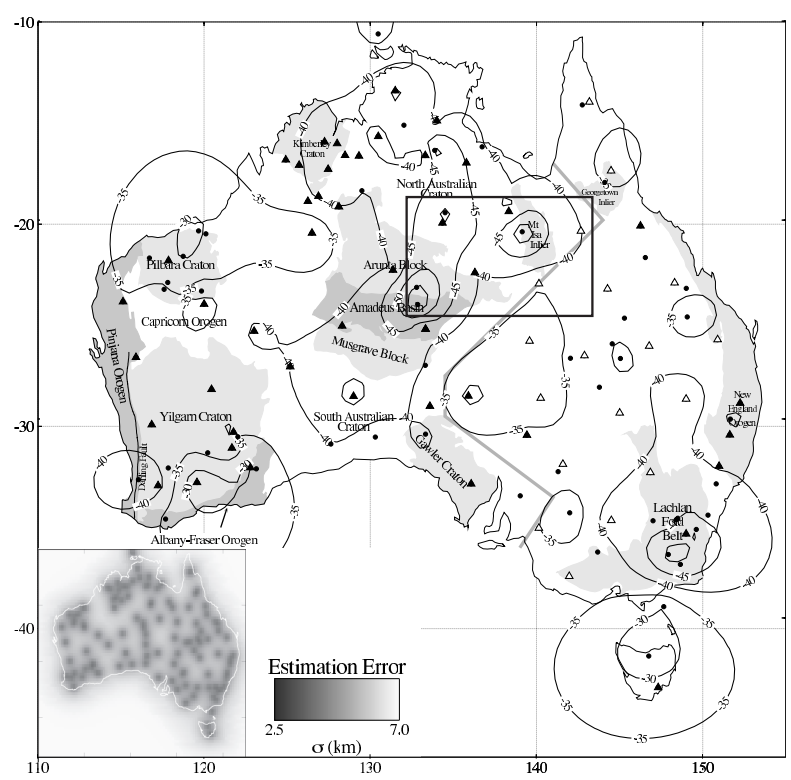

Figure 3.3: Moho depth contour map for the Australian continent underlain by mayor geological structures. The symbols represent single measurements. Inlet illustrates estimated errors. The gray line indicates the transition from thick crust under central Australia to thinner crust in the east. Modified from Clitheroe (1999).

not simply age-dependent (Simons \& van der Hilst, 2002). For Australia this might be caused by tectonic erosion under the Archean parts of the lithosphere due to their locations at the edges of the continent.

\subsection{Seismic tomography results}

In the last 15 years a number of seismic experiments covering the whole continent were conducted (Kennett et al., 2004; Debayle \& Kennett, 2000; Simons et al., 2002; Fishwick et al., 2005; Simons et al., 1999; Kennett, 2003; Direen \& Crawford, 2003; Debayle \& Kennett, 2003). The data were used to compile seismic velocity models of Australia on the basis of surface and body waves. These allow mapping of seismic velocity in a depth range of 75 to $300 \mathrm{~km}$ and a lateral resolution of about $200 \mathrm{~km}$ (Fishwick et al., 2005). Total velocity variations range from -8 to $+8 \%$ from the base model. Figure 3.4 shows S-wave velocity perturbation models for depths of 100 $\mathrm{km}$ and $200 \mathrm{~km}$. While these models are too coarse to resolve typical crustal scale structures they can be used to define a lower crustal to upper mantle seismological indication for the Tasman line. At $100 \mathrm{~km}$ depth the transition zone between lower wave speeds to the east and faster velocities in central Australia is east of the proposed Tasman lines. With increasing depth the transition zone moves westwards (Figure 3.4). The lateral resolution of approximately $200 \mathrm{~km}$ does not allow an exact location of the transition zone and therefore complicates its identification with any of the proposed Tasman lines (Kennett et al., 2004). For all depths the transition zone and Tasman lines are not in agreement on the Cape York Peninsula. The TasmaL project was started by the Australian National University in 2003 in order to improve path coverage in the central part of the proposed Tasman lines (ANU website, 2005). In general higher velocities can be found under the Proterozoic and 


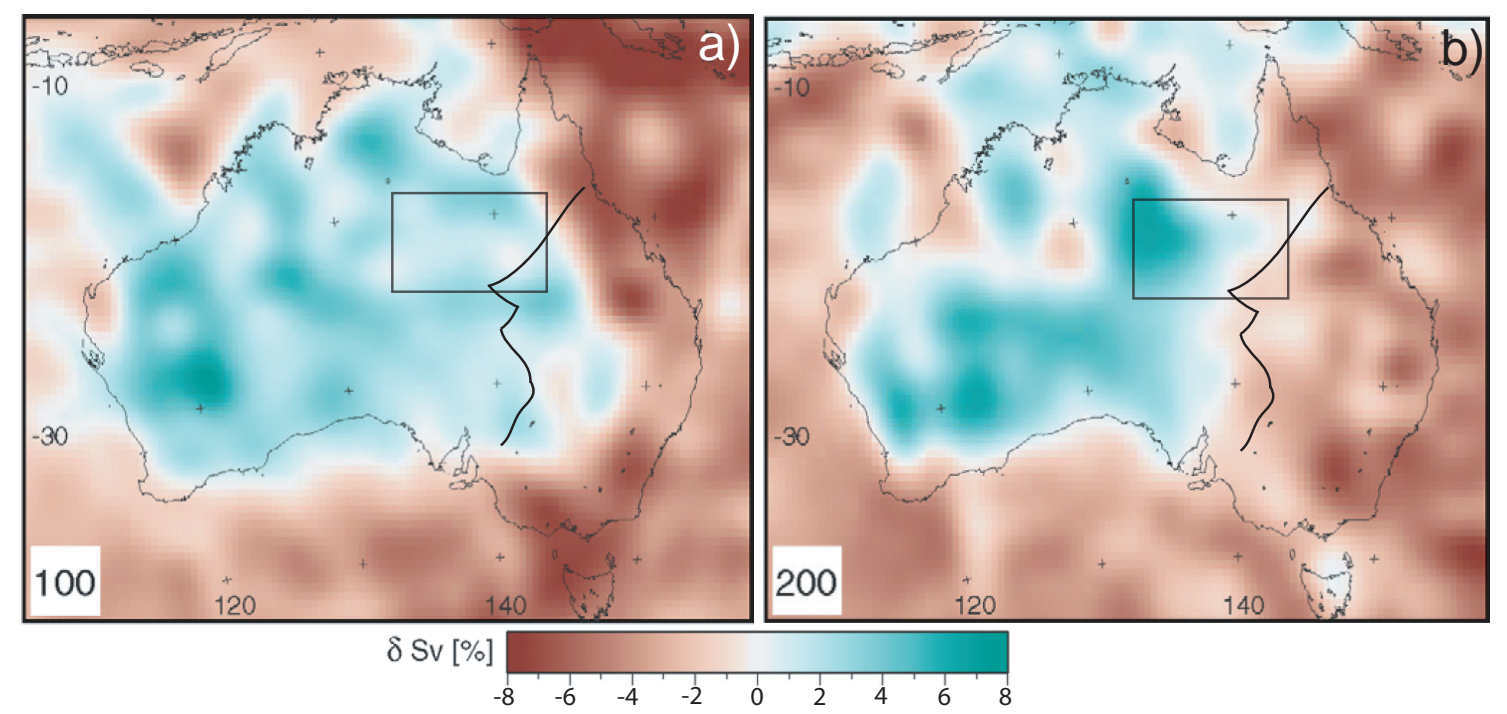

Figure 3.4: Tomographic S-wave models at (a) $100 \mathrm{~km}$ and (b) $200 \mathrm{~km}$ depth. The box indicates the region of our survey. The black line follows the Tasman line as defined by Gunn et al. (1997) (modified from Fishwick et al. (2005)).

Archean parts of the continent.

Simons \& van der Hilst (2003) report seismically anisotropic structures in the mantle below Australia. In the mantle, lattice preferred orientation of olivine crystals along the axis of highest strain (e.g. due to movements of the tectonic plates) is one mechanism to produce seismically anisotropic structures. Simons \& van der Hilst (2003) state that the top 150-200 km of the Australian lithosphere primarily record past deformation processes while below $200 \mathrm{~km}$ the fast direction of the anisotropy matches the current plate motion in the hot-spot reference frame. Heintz \& Kennett (2005) present comparable shear wave splitting results with slightly inconsistent fast directions along the Tasman line possibly indicating regional changes in that area.

\subsection{Gravity surveys}

Since the 1970's a large number of gravity measurements have been conducted all over Australia. Murray (2001) compiled the results in the Australian gravity anomaly map that covers the whole continent. The dataset has a grid mesh of $5 \mathrm{~km}$ while the maximum station spacing is $11 \mathrm{~km}$ (Gunn et al., 1997). Figure 3.5 shows the Bouger anomaly map for our study area. The most prominent feature is an elongated structure of gravity highs accompanied by strong gravity gradients along $140^{\circ}$ east, known as the Barramundi Worm (Bierlein \& Betts, 2004). This structure extends several hundred kilometer further south than the Mt Isa Block as defined by surface geology, possibly indicating that the rocks of the Mt Isa Block continue 


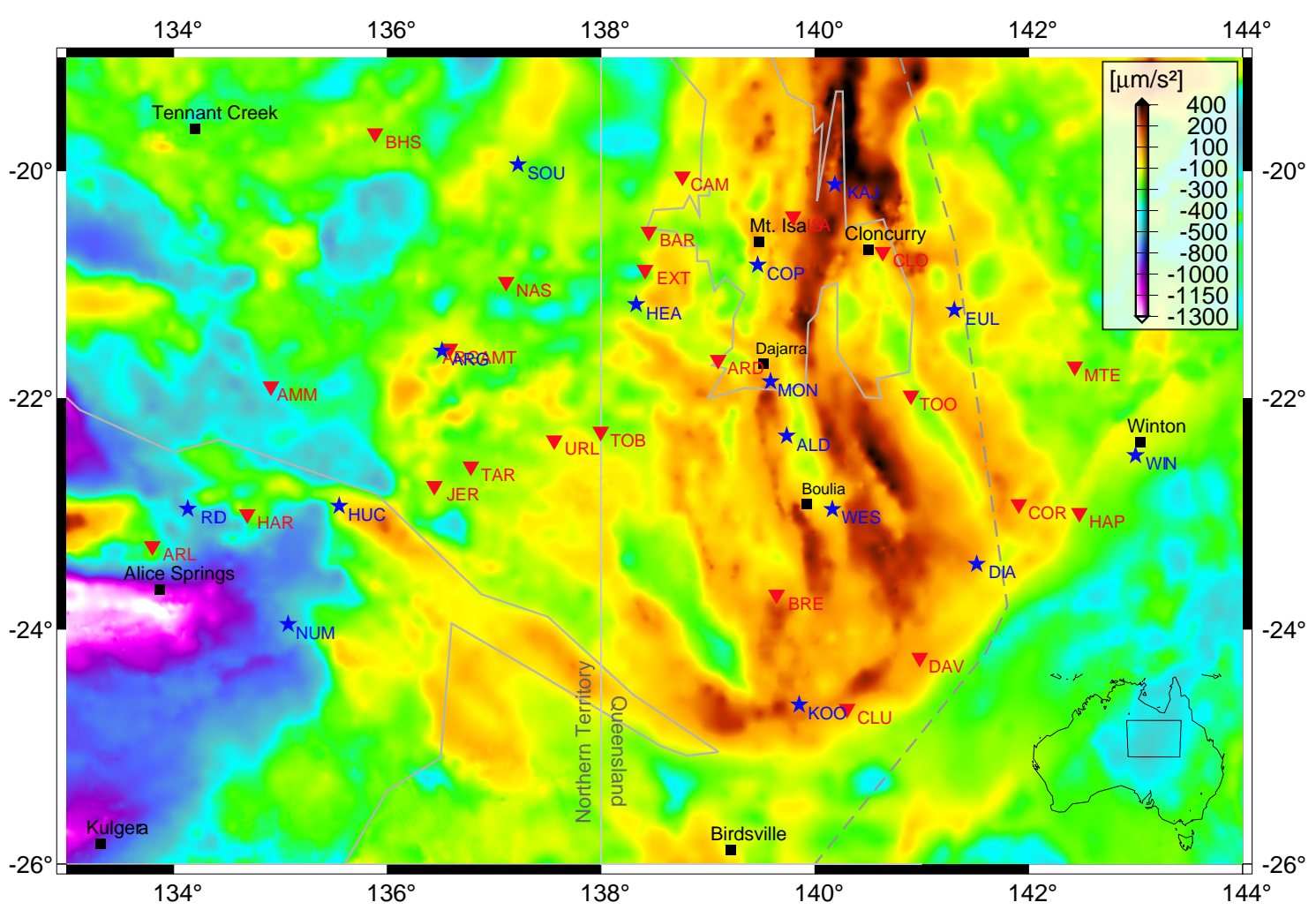

Figure 3.5: Gravity anomaly map of the study area. The symbols indicate locations of MT sites. Arunta Block and Mt Isa inlier are outlined by thick grey lines. The dashed line represents the Carpentaria conductivity anomaly as defined by Chamalaun et al. (1999). Gravity data from Geoscience Australia (Murray, 2001).

under cover for several hundred kilometers (Gunn et al., 1997). Local maxima in vertical gradients of surface gravity fields represent first order boundaries between regions of contrasting density (Bierlein \& Betts, 2004). In this respect the eastern Mt Isa Block and the region south of it can be regarded as such a boundary. Broad gravity highs like the Barramundi Worm can be caused by crustal thinning beneath rifts (Gunn et al., 1997). Therefore the gravity high below the Mt Isa Block could be a remnant of rifting processes active during the Baramundi orogeny (Betts et al., 2002).

Simons et al. (2003) use correlations between gravity anomalies and topography to investigate anisotropic structures caused by fossilized strain fields. Their method uses the direction-dependence of coherence functions for topography-gravity correlations. Depending on the wavelength observed, mechanical anisotropy for shallow $(20-150 \mathrm{~km})$ or greater depth $(>150 \mathrm{~km})$ can be observed. Comparison with directions of seismic anisotropy from SKS-, SKKS- and PKS-splitting shows that for most cases the directions differ by large angles. For depths greater than $200 \mathrm{~km}$ however both methods show similar results in northerly directions, possibly indicating a connection to the present day plate motion. 


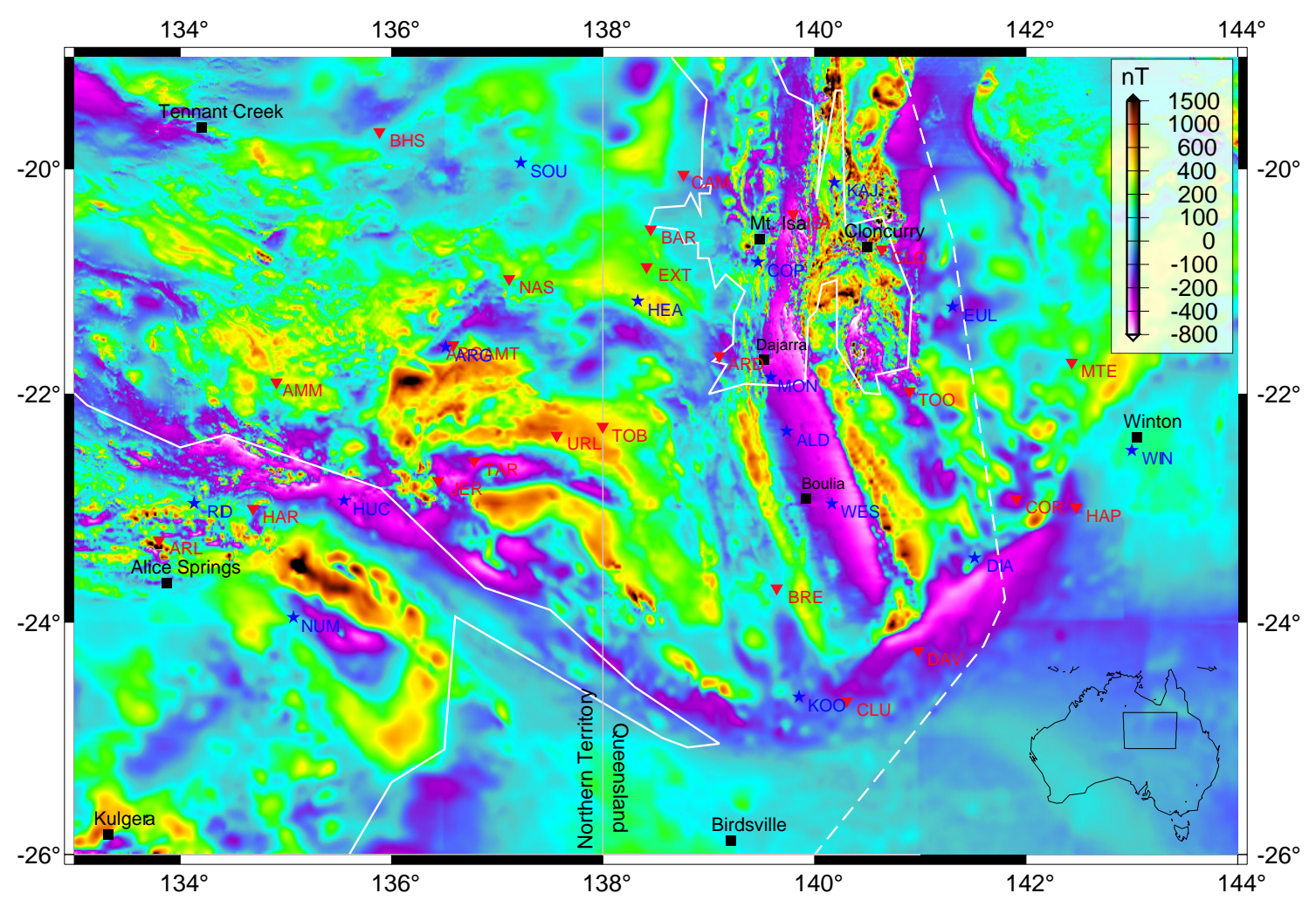

Figure 3.6: Magnetic anomaly map of the study area. The symbols indicate locations of MT sites. Arunta Block and Mt Isa Inlier are outlined by thick white lines. The dashed line represents the Carpentaria conductivity anomaly as defined by Chamalaun et al. (1999). Magnetic anomaly data from Geoscience Australia (Petkovic \& Milligan, 2002).

\subsection{Magnetic anomaly data}

From 1951 onwards aeromagnetic surveys have been conducted in Australia. While earlier measurements were made at altitudes of $150 \mathrm{~m}$ and line spacings of 1 to $3 \mathrm{~km}$ most, lines measured from 1990 onwards were measured with line spacings of $400 \mathrm{~m}$ or less and altitudes of $100 \mathrm{~m}$ or less (Gunn et al., 1997). Petkovic \& Milligan (2002) compiled all lines to the magnetic anomaly map of Australia. All data were filtered and corrected for declination and secular variation effects. The grid of the dataset is 15 seconds of arc while the actual resolution depends on the surveys available for the region of interest. Figure 3.6 shows the magnetic anomaly map of our survey area. The map shows strong variations from negative to positive magnetic anomalies in the Mt Isa Block and south of it. The shape is very similar to the Barramundi Worm found in gravity anomaly images (Figure 3.5). Most magnetic rocks posses a remanent magnetization that is determined by the magnetic field during their formation (e.g. during the cooling down of the material, or sedimentation). Thus areas with magmatic origins often show distinct magnetic anomalies. Therefore the strong magnetizations along 140E (Figure 3.6) give additional weight to the rifting 


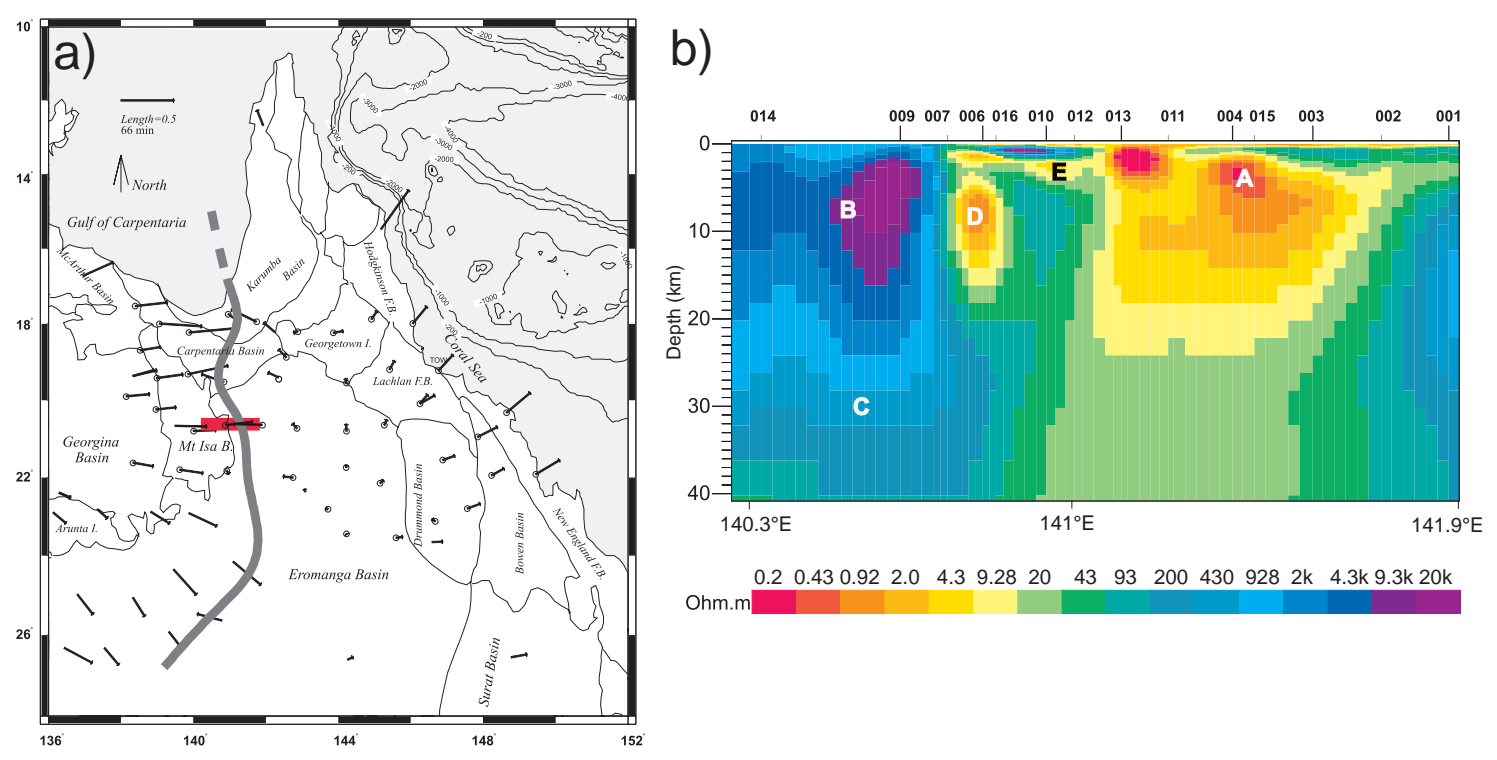

Figure 3.7: (a) Induction arrow response in Queensland. The grey line indicates the location of the Carpentaria conductivity anomaly, the red line the position of the MT profile. (b) 2D inversion results for the MT profile. Figures modified from Lilley et al. (2003).

hypothesis as the formational event for the Mt Isa Block.

In the geologically defined Mt Isa Block higher resolution aeromagnetic surveys have been conducted (indicated by the higher resolution in the area in Figure 3.6). This was for mining purposes as mineral deposits are often associated with magnetic anomalies (Gunn \& Dentith, 1997).

\subsection{Previous electromagnetic induction results}

The first induction surveys conducted in Australia were magnetometer array studies investigating regional structures by induction arrow (2.24) interpretations (Woods \& Lilley, 1979, 1980). This lead to the Australia-Wide Array of Geomagnetic Stations (AWAGS) experiment (Chamalaun \& Barton, 1993) which covered the whole Australian continent with site spacings of about $275 \mathrm{~km}$. The induction arrow responses were interpreted in terms of conductive band-like structures possibly coincident with major structural boundaries (Figure 6.6). In south-western Queensland Woods \& Lilley (1980) found indications for a north-south striking conductive band whose existence was emphasized by the AWAGS data. In order to clarify the position and existence of this so called Carpentaria conductivity anomaly additional magnetometers with smaller site spacings were deployed in 1995 (Chamalaun et al., 1999) fixing the anomaly to the location marked in Figure 3.7. The Carpentaria conductivity anomaly is a major structure in the Australia-wide thin-sheet conductivity model of Wang \& Lilley (1999). In order to investigate the possible depth extend of the Carpentaria conductivity anomaly Lilley et al. (2003) measured an MT profile 
perpendicular to the anomaly (thin yellow line in Figure 3.2). Their 2D inversion results (Figure 3.7b) show very resistive basement in the Mt Isa Block (1000-10000 $\Omega \mathrm{m}), 300 \mathrm{~m}$ thick sediments over the Eromanga basin $(\approx 1 \Omega \mathrm{m})$ and the conductivity anomaly extending from $300 \mathrm{~m}$ to $25 \mathrm{~km}$ depth $(0.2-10 \Omega \mathrm{m})$. The width of the anomaly is $20-30 \mathrm{~km}$. In conjunction with the good agreement with magnetic anomaly (Figure 3.6) and gravity (Figure 3.5) data Lilley et al. (2003) interpreted the Carpentaria conductivity anomaly as the eastern boundary of the Mt Isa Block at a plate suture. A possible relation of the anomaly to the further east assumed Tasman line is discussed in Chapter 6.

\subsubsection{Other magnetotelluric studies}

Magnetotelluric (MT) surveys in northern Australia began in 1978. Cull (1982) measured MT profiles in the McArthur Basin (Figure 3.1) north of the Mt Isa Block. While the McArthur Basin is slightly younger than the Mt Isa Block (1.7-1.4 $\mathrm{Ga}$ ) the same back-arc extension might have lead to its creation. Therefore common conductivity structures might be observable. Cull (1982) found typical resistivities of $300 \Omega \mathrm{m}$ to several $1000 \Omega \mathrm{m}$ for the basement rocks while areas covered with sediments were found to be $10 \Omega \mathrm{m}$.

Spence \& Finlayson (1983) investigated the resistivity structure of the Eromanga and the underlying Cooper and Adavale Basins (about $500 \mathrm{~km}$ south-west of our survey area). For the upper $2 \mathrm{~km}$ they were able to use resistivity data from borehole measurements to reference their MT results. For the Eromanga Basin they measured resistivities of 1-7 $\Omega \mathrm{m}$ while the basement was found to be $750-3800 \Omega \mathrm{m}$.

Simpson (2001) conducted wide-spaced long-period MT measurements east of the present study area in order to investigate a possible connection between electrical anisotropy in the upper mantle and plate-tectonics. While electrical anisotropy directions correlate well with the plate motion in a no net rotation frame (Simpson, 2002) the mechanisms leading to the anisotropy are still ambiguous (Simpson \& Tommasi, 2005). The wide station spacing prevents the Simpson (2001) dataset from investigations of regional crustal structures.

Recently Heinson \& White (2005) presented results of seafloor MT measurements in the shallow $(<60 \mathrm{~m}$ water depth) Gulf of Carpentaria. Below a sedimentary cover of some $1000 \mathrm{~S}$ (1-100 $\Omega \mathrm{m}$ for the top $10-20 \mathrm{~km})$ they found basement resistivities of up to $10000 \Omega \mathrm{m}$. An observed phase splitting in the MT results for upper mantle depth could either be related to anisotropy in the lower crust and upper mantle or as preferred by Heinson \& White (2005) due to laterally increasing thickness of the conductive upper crust towards the east. As tidal effects biased the magnetic field data at most sites induction arrows results are contaminated and could not be estimated for all sites. Therefore they cannot clarify if the Carpentaria conductivity anomaly extends in the Gulf of Carpentaria as assumed by Chamalaun et al. (1999). 


\section{Field measurements in central Australia}

In order to investigate possible correlations of electric anisotropy in the upper mantle and absolute plate motion direction of the Australian plate Simpson (2001) conducted magnetotelluric measurements in central Australia. As only six sites were measured at that time a new campaign that extends the earlier measurements to the east was performed between July until October 2003. The moderate climate and small precipitation probability in these month provide good conditions for field measurements in central Australia. Especially the hot temperatures and large daily temperature variations could otherwise affect equipment, data and experimenter while strong rainfall that are more likely late in the year could lead to impassable dirt roads.

The topographic map (Figure 3.2) in the previous chapter shows the location of the measurement sites of the 2003 survey. As the main target of the survey are mantle structures a sparse site spacing of about $200 \mathrm{~km}$ is sufficient to cover large areas, while long measuring times are required to provide long period data of excellent quality. Operation times of 6-8 weeks were chosen for these sites (marked as blue stars in Figure 3.2). In order to investigate crustal structures, the main topic of this study, a more dense site spacing is required to avoid spatial alias. Therefore additional sites were measured during the servicing trips of the long period sites. For logistic reasons these sites operated for 12-16h (over night) only. They are marked as red inverted triangles in Figure 3.2 and lead to a site spacing of roughly 50 kilometers. The locations and operating times of all sites are shown in Appendix A.1.

The equipment used at all sites were Göttingen type RAP data loggers (Steveling \& Leven, 1992) with Magson fluxgate magnetometers and $\mathrm{Ag} / \mathrm{AgCl}$ electrodes. Five channels, two horizontal electric and three magnetic field components were recorded in the geomagnetic coordinate system. Ten instruments were used in total, nine recorded at long period sites while the tenth was used for the overnight measurements. The fastest sampling rate was 2 seconds leading to a shortest available period of 8 seconds. The resulting frequency for the long period sites range from 8 to $\approx 30000$ s. As overlapping time intervals exist for nearly all sites, MT and GDS transfer functions could be calculated for most sites. GPS receivers were used for correct timing between the sites. For both MT and GDS a remote reference (Gamble et al., 1979) processing from the University of Göttingen was used (more details in Simpson \& Bahr (2005) or the Appendix of Hanekop (2003)). The results were compared with processing results of the current version of the robust remote reference 

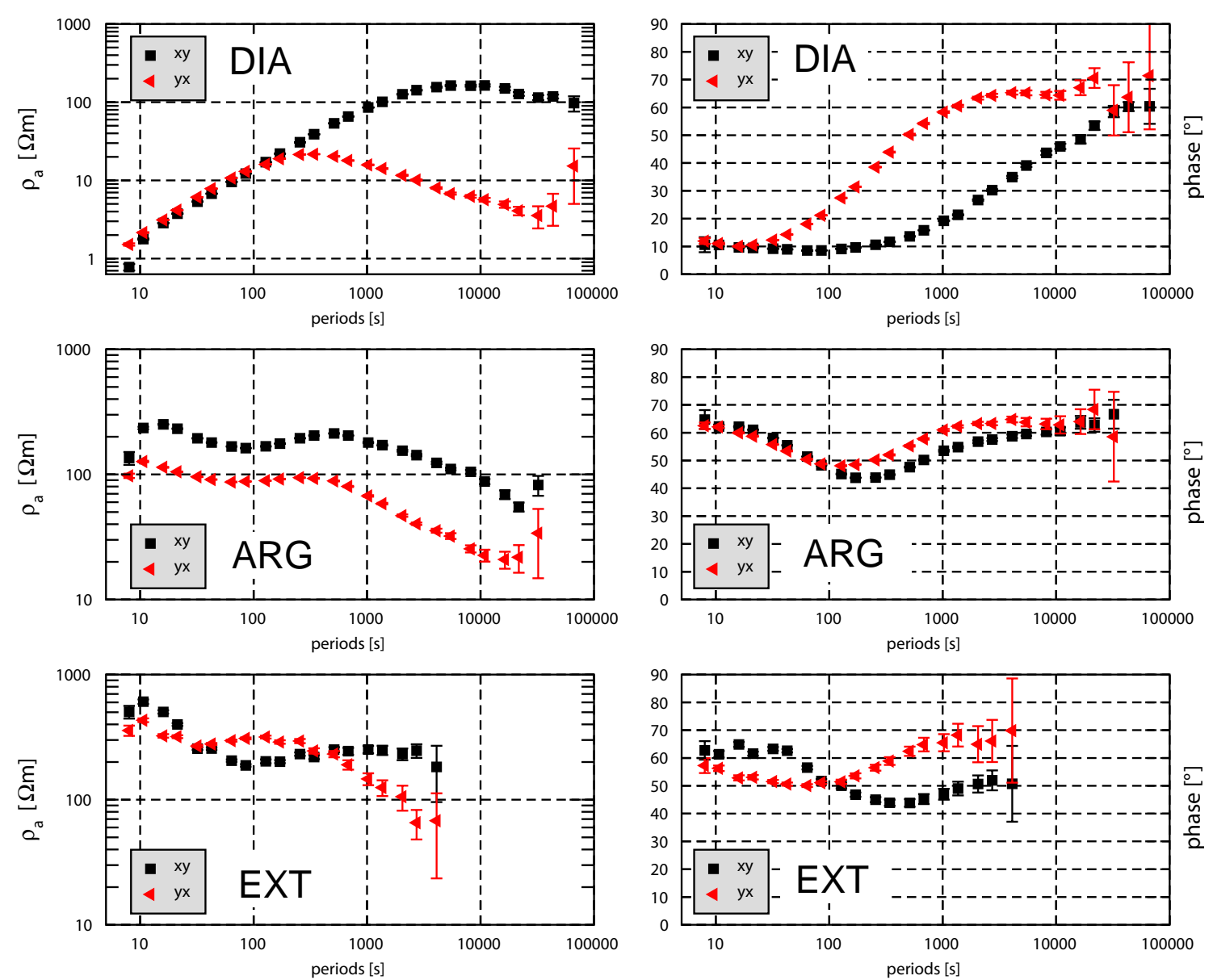

Figure 4.1: Apparent resistivity and phase curves for sites DIA, ARG and EXT. Site ARG is exemplary for the western part of the array while DIA is typical for the long period sites in the east. Site EXT shows typical resolution for sites measured overnight only.

processing code of Egbert \& Booker (1986). Due to the low noise conditions in the remote areas of central Australia no significant differences between both processings could be observed.

The following Chapter presents the MT and GDS datasets measured in central Australia and discusses first implications from the data. A more sophisticated interpretation including the development of a conductivity model of the region will follow in Chapter 6.

\subsection{MT results}

Figure 4.1 shows MT apparent resistivities (2.28) and phases (2.29) for the long period sites ARG and DIA in an unrotated coordinate system. These two sites are typical for the western respectively eastern part of the array. The apparent resistiv- 
ities and phases at site ARG show only small coordinate system dependence. The underlying structures, primarily the Georgina Basin (Section 3.1), are approximately one dimensional (1D) with slightly decreasing resistivities with depth. Therefore this site is used as the reference site for the GDS method. Site DIA in the eastern part of the array shows very low apparent resistivities and phases at short periods and a strong phase split between the xy and yx components for periods longer than 100s. This behavior is typical for the easternmost sites and is related to the conductive sediments of the Great Artesian Basin and the Carpentaria conductivity anomaly (Section 3.6). The data of site EXT in Figure 4.1 indicates the data quality that could be achieved from the additional overnight measurements. As usual for short measuring times in low noise conditions these sites show good data quality from 10 to about 1000 seconds (Hanekop \& Simpson, 2006). The measuring time of only half a day provides only a small set of estimates that can be used in the weighted stacking procedures of MT processing. Therefore the obtainable data quality strongly depends on the geomagnetic activity during the measurements. Weak source fields lead to low signal to noise ratios and thus to larger errors in the data. This effect is evident at some sites (e.g. TOB, TAR, see Appendix A.2) where reasonable results were obtained for a period range from 64-683s only. Apparent resistivity and phase curves of all sites are plotted in Appendix A.2.

Lateral changes in the crustal structures can be visualized by contour plots of the apparent resistivity and phases for one period. As these plots interpolate (with spline interpolations or triangulation) between the field sites some pitfalls of this visualization have to be considered.

- spline interpolations use polynomial functions. Therefore overshoots are possible in between sites

- the lack of data outside the outermost field sites lead to undetermined results outside of the target area

- site distances larger than the penetration depth of the investigated period might lead to spatial alias problems. At best the interpolation ignores unresolved structures between the sites, in worse scenarios local structures below field sites are blurred to regional structures or the alias problem leads to artefact structures between the field sites.

- Due to the inductive nature of MT transfer functions the penetration depth depends on the local conductivity structure. Visualization of one period can therefore lead to interpolations between structures of different depth.

- Elongated 2D and 3D structures lead to a strong influence of the coordinate system the impedance tensor is visualized in. For a contour plot one coordinate system that is adequate for most sites has to be chosen. Plotting sites with significantly different strike angles in the wrong coordinate system might lead to artifacts or vanishing structures when only the off-diagonal impedance tensor elements are observed (note that even an undistorted 2D tensor (2.33) 
rotated by $45^{\circ}$ is indistinguishable from a $1 \mathrm{D}$ tensor when only the off-diagonal elements are investigated). This problem can be overcome by the observation of rotational invariants.

Some of these pitfalls can have severe impact on the interpretation of the field data. Careful considerations of the behavior of every single site however can lead to good results. For this study the major difficulties with interpolation procedures can be overcome as (i) the distance between field sites appears to be smaller than the average size of the crustal structures and (ii) the strike angle investigations in the following section indicate a predominant strike in northerly respectively easterly directions. Plots of the apparent resistivity have to be handled with special care as static shift effects (Section 2.3.3) might have severe influence on the apparent resistivities.

Figures 4.2 and 4.3 show contour plots of the apparent resistivities and phases for all available MT sites and four periods between 16 and 1024 seconds. Only the results of the off-diagonal elements of the impedance tensor are plotted in an unrotated $(\mathrm{N}$ S, E-W) coordinate system. Data of all sites marked by red stars have been used for the plots. For $16 \mathrm{~s}$ the phases of both components show very low values in the eastern part that correspond to the low resistivities of the Great Artesian Basin sediments that cover more resistive material. High phases above the Mt Isa Block indicate high resistivities in the upper kilometers of the crust with increasing conductivities with depth. For longer periods strong differences between the two components occur. In the xy component the low phases in the east reaches further north while the high phases above the Mt Isa Block increase in lateral extend. While both effects could be explained in general with the Carpentaria conductivity anomaly and a larger subsurface extend of the Mt Isa Block than that inferred from surface geology (plotted in the Figures) a quantitative interpretation requires 3D modeling (Chapter 6). Basically equal phases in both components (at least until 256s) in the western part of the array indicate approximately 1D upper crustal structures in that area. The phases at sites KAJ and EUL (see Figure 3.2 for locations) show phases above 90 degrees for some periods in strike direction. These are indicators for complex 3D structures (Lezaeta \& Haak, 2003) and can be created by (i) bended bandlike conductivity structures (ii) anisotropic structures with different directions (Heise \& Pous, 2003), (iii) multiple 2D structures with differing strike directions (Weckmann et al., 2003) or (iv) a fractal system of interconnected high conductive phases (fluids or graphite) (Bahr, 1997, 2000).

The apparent resistivities in Figure 4.3 support the statements inferred from the phases. Especially in the eastern part where very low resistivities of 1-10 $\Omega m$ are observed at nearly all sites static shift effects seem to be negligible as it is unlikely that all sites and polarizations are effected by the same distortion effects. For longer periods however it is possible that galvanic effects associated with the Carpentaria anomaly influence the measured impedance tensors. The apparent resistivities in the western part of the array are significantly larger than those in the east. Some of the sites show static shift effects indicated by remarkably different apparent resistivities for the two polarizations without strong signatures in the phases. This 
was already evident in Figure 4.1 where site ARG had statically shifted apparent resistivities over the whole period range. The fact that the western part of the array is effected by static shift while the eastern part is basically unaffected is related to the shallow conductivity structures. Static shift is often caused by small scale conductive rather than resistive structures as these usually provide stronger resistivity gradients (Section 2.3.3). Therefore strong small-scale conductivity gradients are more likely in the resistive western than in the conductive eastern part of the array.

The fact that the apparent resistivities and especially the phases show good correlation between neighboring sites emphasizes the assumption that the main crustal structures in the survey area have a greater extend than the site spacing of about $50 \mathrm{~km}$ and are therefore resolvable by our measurements. While this is a remarkable result in the more resistive western part of the array this is not surprising in the eastern part where the conductive sediments in the near surface obscure possible small scale resistive structures at greater depth.

\subsubsection{Strike angles}

The impedance tensor for multidimensional structures is coordinate system dependent. For elongated structures it is often possible to approximate the structure as $2 \mathrm{D}$ in a certain frequency (depth) range. For these and purely 2D structures several methods have been developed to recover the strike direction of the structure (Sections 2.3.2, 2.3.3 + 2.4). While these procedures generally lead to a result the validity of the assumptions that have been made has to be checked (see Section 2.3.4). Figure 4.4 (right part) plots Bahr (1988) decomposition angles (equation 2.40) and skew values (equation 2.41). The strike direction is chosen to maximize the phase of the xy polarization and thus points in strike direction above conductive structures (perpendicular for sites laterally removed from the conductive structure) or in the conductive direction for anisotropic structures at depth. The size of the boxes indicates the skew values while the color codes the skew in three classes (green) smaller 0.1, (yellow) smaller 0.3 and (red) larger than 0.3. For skew values larger than 0.3 the structure cannot be regarded as $2 \mathrm{D}$ while smaller values do not necessarily mean that the structure actually is $2 \mathrm{D}$ (Section 2.3.3). The length of the lines indicates the phase difference of the xy and yx polarizations in strike coordinates and therefore illustrates deviations from 1D structures where no phase difference exists.

For short periods the strike direction in the Mt Isa Block is roughly North-South, corresponding to the predominant major fault zones in the region (Bierlein \& Betts, 2004). For the western part phase differences are small and the strike directions meaningless as they are related to small scale structures that are not resolvable with the present site spacing. In the eastern part the strike directions of sites close to the Carpentaria conductor (dashed line in the Figures) are in agreement with the direction of the anomaly (note the 90 degree uncertainty depending on the location of a site on or next to an anomaly or regional conductivity contrast). The skews in the eastern part are higher than in the rest of the array and generally higher in 


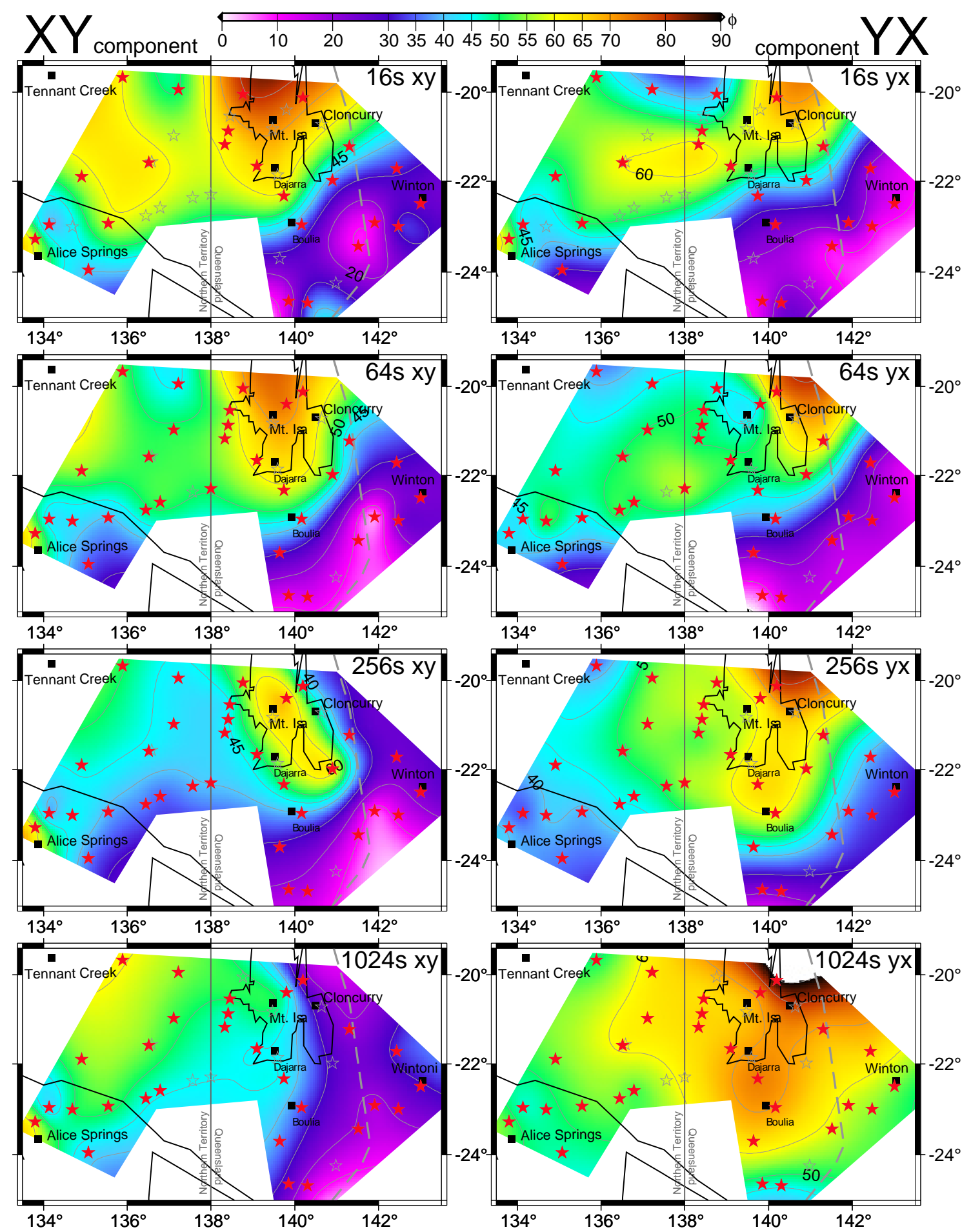

Figure 4.2: MT-phases (xy and yx components) for four periods. Spline interpolations were used to create the contour plots. Red stars indicate stations used for interpolation. 


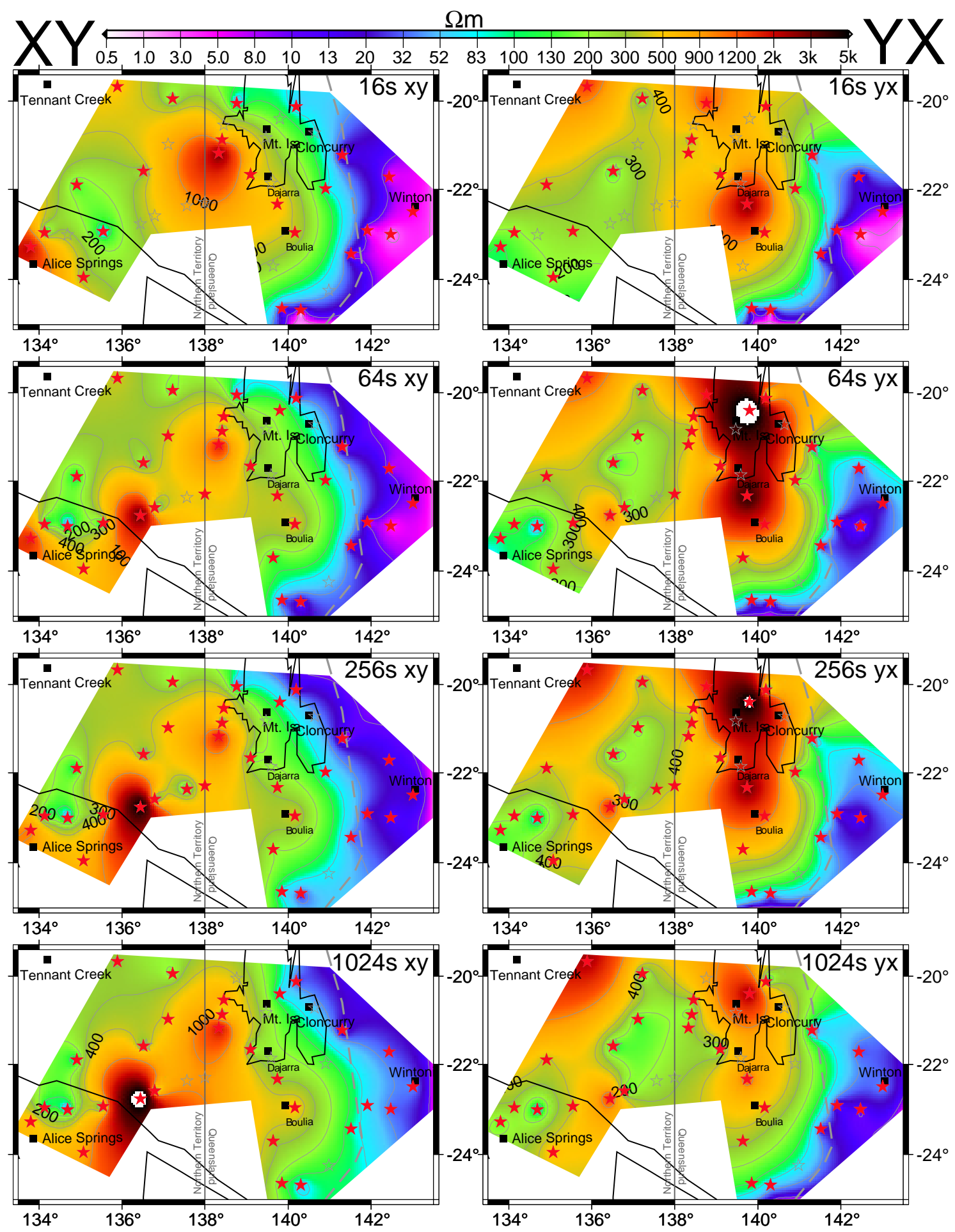

Figure 4.3: MT-apparent resistivities ( $\mathrm{xy}$ and $\mathrm{yx}$ components) for four periods. Spline interpolations were used to create the contour plots. Red stars indicate stations used for interpolation. 


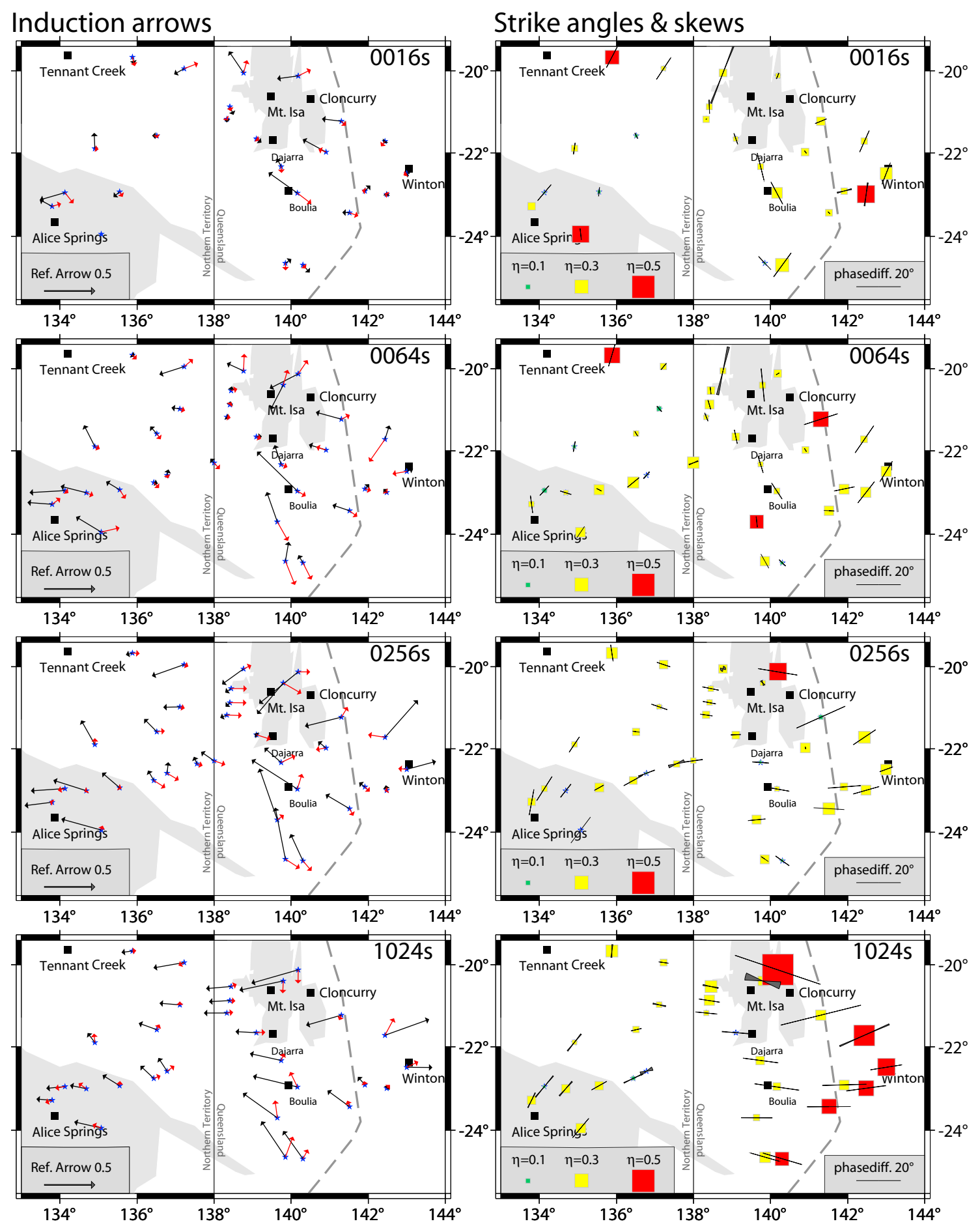

Figure 4.4: Left: Real (black) and imaginary (red) local induction arrows for 4 periods. Right: Bahr's strike angles and skew values $\eta$. The length of the lines indicates the phase difference between xy and yx polarization in strike coordinates while the square size scales with the skew. Square colors ranks the skew in 3 classes: green $(<0.1)$, yellow $(0.1$ to 0.3$)$ and red $(>0.3)$. 
regions with strong conductivity gradients. For conductive band-like structures such as the postulated Carpentaria anomaly this implies that bends in the structure can lead to high skew angles although the structure is two-dimensional in first approximation. Alternatively the depth and extension variations of the Eromanga basin could lead to 3D effects.

From 256 seconds onwards the strike directions in the whole area become more coherent. An easterly trend that turns towards the south in the westernmost part of the array becomes visible. Especially in the eastern part this is associated with a significant increase in the skew values that indicate three-dimensional effects. At the same time the phase differences increase. For periods above 1024 seconds this could be related to anisotropic structures in the mantle (Simpson, 2001) that are investigated by a different project. Other explanations range from large scale 2D anomalies to conductive cracks in the crust that are aligned with the crustal stress field (Hillis \& Reynolds, 2003; Reynolds et al., 2005).

The phase sensitive skews in the eastern part increases with period. Therefore the deviations from $1 \mathrm{D}$ or $2 \mathrm{D}$ structures originate at greater depth. Otherwise the skew would decrease for longer periods. Extreme skew values at site KAJ are related to $3 \mathrm{D}$ structures that were already indicated by phases above 90 degrees (Figure 4.2) that are usually accompanied by high skews.

\section{Magnetic distortion analysis}

As discussed in Chapter 2.4.1 current channeling (Jones, 1983) in conductive structures might lead to magnetic distortion (Chave \& Smith, 1994). The high skew values and increased $d_{D}$ GDS perturbation tensor elements (see Chapter 4.2) in the eastern part of the array are possibly related to magnetic distortion produced by current channeling effects in the Carpentaria conductivity anomaly. Exemplary for the highly distorted eastern sites Figure 4.5 plots the strike angles of different decomposition schemes for site EUL. The results from Swift's method are compared to the galvanic distortion only methods of Bahr (1988), Groom \& Bailey (1989), Becken \& Burkhardt (2004) and the electric and magnetic distortion decomposition of Smith (1997). For the latter method three neighboring frequencies were used to estimate the strike direction. Next to the strike direction the RMS misfit for the least square approaches and the skew values of Swift's and Bahr's methods are plotted. A normalized RMS of 1 corresponds to a fit within the data errors. While Swift's method obviously leads to false strike directions that are influenced by distortion effects the other methods agree quite well. Despite matching strike angles of the different methods, high skew values and large RMS for periods up to several 100 seconds indicate that the shallow structures cannot be regarded as two-dimensional. The small RMS of Smith's method at short periods is related to a larger amount of parameters used for the fit. In general no significant difference between Smith's and the galvanic distortion only decompositions can be observed. This is related to negligible magnetic distortion tensor elements in Smith's decomposition of site EUL. In that case magnetic distortion becomes irrelevant and Smith's decomposition sim- 


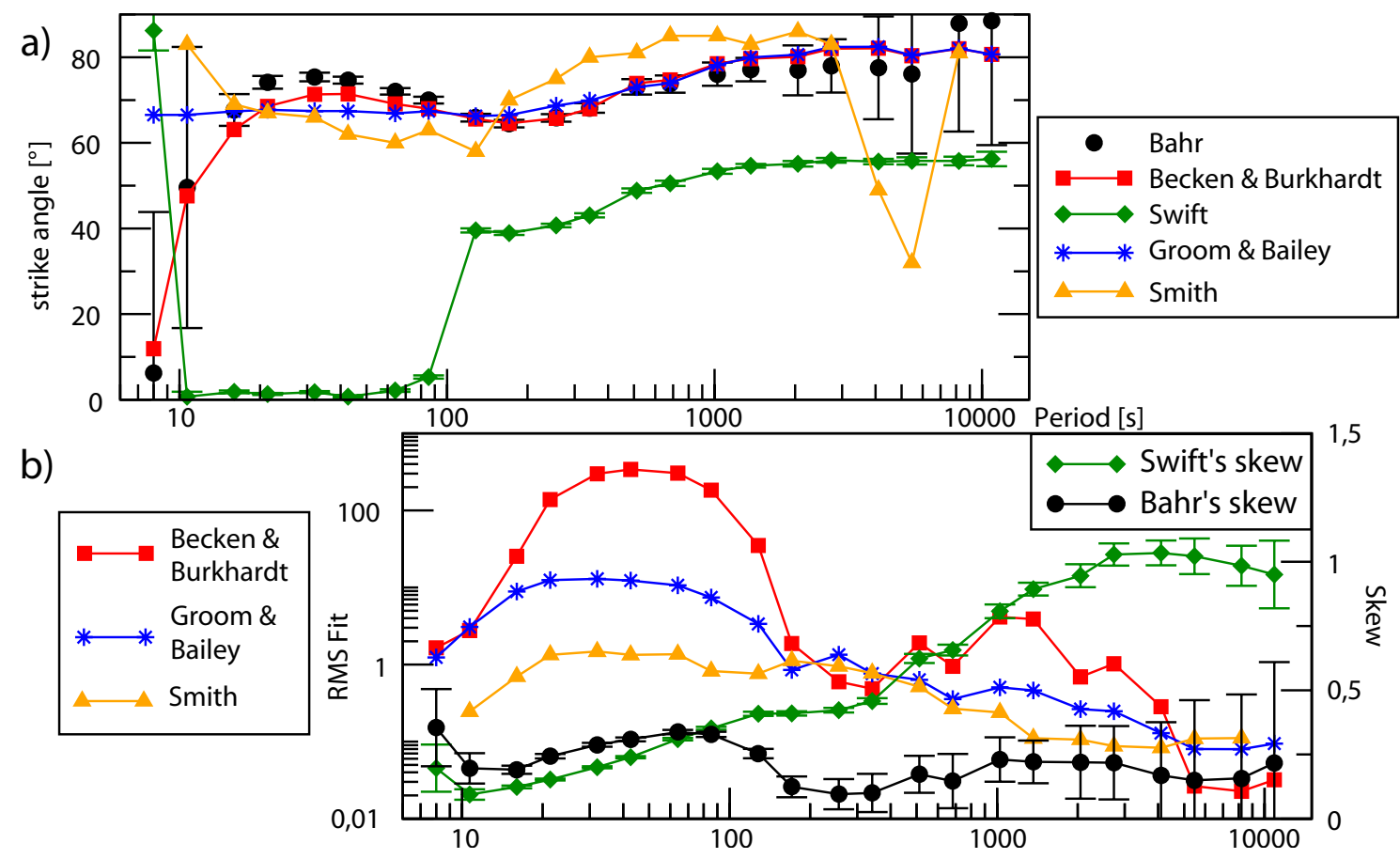

Figure 4.5: (a) Period dependent strike direction of different methods for site EUL. For Smith's method 3 neighboring frequencies have been used for the fit, all other results are single frequency estimations. (b) RMS misfit for least square approaches and skew values for Bahr's and Swift's method.

plifies to that of Groom \& Bailey. As Smith assumes frequency independent, real valued magnetic distortion it is possible that complex valued magnetic distortion that is inextricably mixed with the complex regional impedances affects the data. This matches with numerical studies of Agarwal \& Weaver (2000) that showed that magnetic distortion parameters are complex valued at inductive depth. For longer periods the magnetic distortion parameters are real valued but their amplitudes become insignificant.

Similar investigations at the other sites showed that no magnetic distortion effects in the manner of Chave \& Smith (1994) could be found in the Australian dataset. For the western part of the array with its approximately 1D shallow structures this should be expected. For the sites in the East however large skew angles along the presumably bandlike Carpentaria anomaly indicate multidimensional structures. This obvious contradiction can be explained with bends and variations in width of the bandlike structure. These can produce severe distortions and 3D effects, particularly when the bandlike structure is significantly more conductive than its surrounding. This is the case where the Carpentaria anomaly meets the resistive Mt Isa Block. As the electric field is more sensitive to such 3D effects than the magnetic field, magnetic transfer function may lead to simpler interpretations of elongated structures. This will be investigated in the following. 


\subsubsection{Local induction arrows}

Real and imaginary local induction vectors (equation 2.24) indicate lateral conductivity gradients with real parts pointing away from conductive structures in the Wiese (1962) convention. The amplitude scales with the distance from the boundary between conductive and resistive material and the amplitude of the conductivity gradient (Section 2.2.2). Real and imaginary induction vectors are not independent. While the real part indicates the strength of the vertical magnetic field the imaginary part illustrates the change in the field and thus the change in the real induction vector. The left column of Figure 4.4 plots real and imaginary induction vectors for periods between 16 and 1024 seconds. For 16s the induction vectors show nonuniform patterns indicating the influence of small scale structures that are unresolvable with the present site spacing. For longer periods $(>64 s)$ arrows of sites close to the Carpentaria conductivity anomaly start pointing away from the anomaly as found by Chamalaun et al. (1999). With increasing induction space, at periods greater $256 \mathrm{~s}$, the pattern becomes more consistent reaching maximum induction arrow length between 256 and 1024 seconds. The boundaries of the crystalline Mt Isa and Arunta blocks as defined from surface geology (grey shaded in the figures) have no significant influence on the induction arrows.

The behavior of the sites close to the Carpentaria conductivity anomaly matches well with the idea of a conductive band. The arrows are pointing away from the conductor, the maximum amplitudes are observed just off the band and the vectors vanish directly above the structure. The sites in the western part of the array however are too far removed from the anomaly to attribute their westerly arrow directions to the Carpentaria anomaly. Wang \& Lilley (1999) and Lilley et al. (2003) nevertheless argue that the induction arrows are related to the Carpentaria conductor. In fact an extremely conductive bandlike structure in a very resistive surrounding can produce significant induction arrows at great distances. For the present case however this is unlikely for three reasons: (i) the apparent resistivities of sites west of the Mt Isa Block range around 200-500 $\Omega m$ which would be too conductive for a strong influence at sites $500 \mathrm{~km}$ away from the anomaly, (ii) the induction arrows in the western part already point westwards at $64 \mathrm{~s}$ where the induction space is too small even when extremely resistive structures would be present and (iii) between the westernmost sites and the anomaly lies a region that shows minimal induction vectors. The lateral consistency of the induction arrows however suggest a common origin of the induction arrow pattern. In order to test the consistency between the observed induction arrows and the MT and GDS results, conversions of the different measures are performed in Chapter 5. Alternatively source field inhomogeneities in conjunction with the wide-stretched survey area could lead to large scale induction arrow patterns but the good agreement of our results with the induction arrows of previous measurements suggests variations in the conductivity structures as sources of the longer period induction arrows.

The induction arrows have implications for the conduction mechanisms that lead to the $2 \mathrm{D}$ strike directions of the previous section. For $2 \mathrm{D}$ conductive structures the 
induction arrows would point perpendicular to the strike direction. Homogenous anisotropic structures however do not create vertical magnetic fields and thus no induction arrows. Therefore multiple heterogenous 2D or anisotropic structures are required to produce both, the observed MT strike directions and the induction arrow patterns.

\subsection{GDS results}

Ten identical instruments, nine permanently installed and one used for the overnight sites, were used for the field measurements in central Australia, providing excellent conditions for the calculation of GDS transfer functions. Long overlapping measuring times for the long periods sites provide high quality GDS transfer functions between these sites. This allows a change of reference site if necessary. For the overnight measurements any of the long period sites can be used as reference.

Due to their excellent data quality sites DIA and HUC were chosen as reference sites for the processing of the first and second part of the campaign respectively (see Appendix A.1 for recording times of the sites). According to section 2.2.1 the final reference site should (i) provide good data quality and (ii) for simplicity of the interpretation of the magnetic anomalies be located above approximately 1D conductivity structures. While site DIA is a good choice in terms of running time and data quality obvious multidimensional structures make it unfavorable as final reference site. Therefore site ARG, the site with the smallest deviations from a 1D structure has been chosen as final reference site. The program gdsswap (see Appendix B.2.1) was used to perform the reference site transformations.

Figure 4.6 shows contour plots of the $d_{D}$ component of the perturbation tensor for periods from 43-2732 seconds in an unrotated coordinate system. Note that due to the coordinate system dependence all problems of interpolated contour plots discussed in Section 4.1 remain valid for the perturbation tensor elements. Assuming 1D structures at the reference site, increased $d_{D}$ amplitudes indicate increased magnetic fields in east-west direction. For negligible $h_{H}, h_{D}$ and $d_{H}$ components this correlates with increased current densities in northward direction and thus a north-south striking conductivity anomaly. For short periods $d_{D}$ vanishes in most parts of the array (Figure 4.6). Only in the eastern part, along the Carpentaria anomaly significantly increased amplitudes are observed. Maximum amplitudes are observed at site EUL that shows strong $3 \mathrm{D}$ effects (phases $>90^{\circ}$ ) in the MT data. While the amplitudes in the western part remain negligible for longer periods the $d_{D}$ anomaly in the east increases in amplitude reaching a maximum between 512 and 683 seconds. For long periods the effect diminishes slowly as could be expected for anomalies originating from crustal structures and more homogenous mantle conductivities. All perturbation tensor elements for a wide period range are plotted in Figures A.5 and A.6 in Appendix A.2. These Figures show that the horizontal perturbation tensor elements $h_{H}, h_{D}$ and $d_{H}$ are in fact negligible for most periods. Only at the southern end of the Carpentaria anomaly 3D structures are indicated 


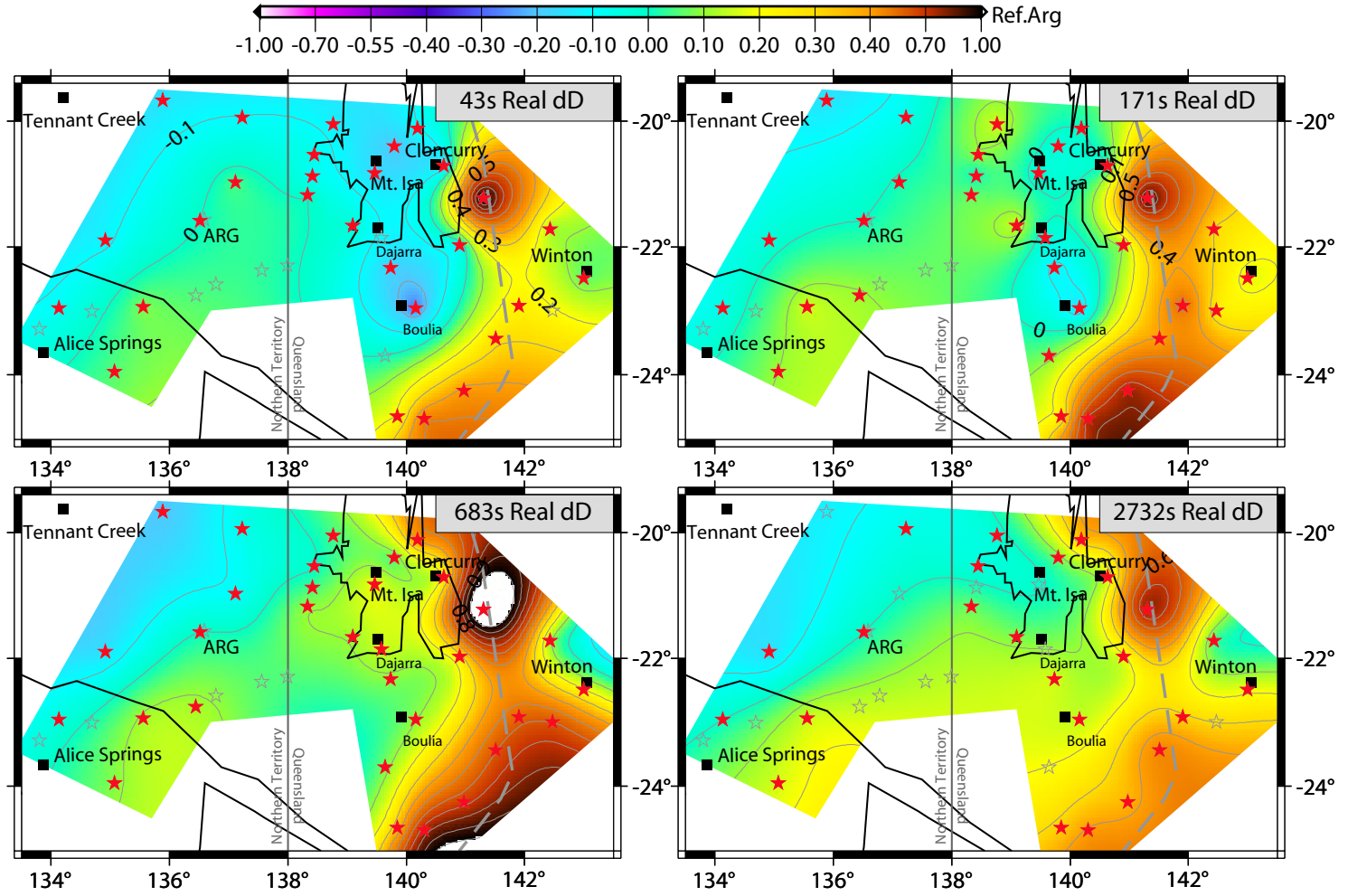

Figure 4.6: Real part of the $d_{D}$ component of the GDS perturbation tensor for four periods between 43 and 2732 seconds using site ARG as reference site.

by increased amplitudes in all elements. These can be explained by a bend in the anomaly that follows the southern end of the gravity (Figure 3.5) and magnetic (Figure 3.6) anomalies (and possibly the Tasman line, Chapter 3).

\section{GDS strike directions}

Figure 4.7 shows GDS strike angles that were obtained by maximization of $d_{D}$ with respect to the other horizontal perturbation tensor elements for a period of 683 seconds (compare Section 2.2). In order to illustrate two-dimensionality the length of the dark grey wedges in the Figure scale with the ratio $\left|d_{D}\right| /\left(\left|h_{H}\right|+\right.$ $\left.\left|h_{D}\right|+\left|d_{H}\right|\right)$. As this ratio might be large even for small $d_{D}$ anomalies ellipses with $d_{D}$ and $h_{H}$ as principal axes are plotted in addition. Elongated ellipses indicate strong anomalies in one direction while small, circle-like ellipses imply weak direction dependent anomalies. The light grey circles in Figure 4.7 scale with the sum of $h_{D}$ and $d_{H}$ which are only non-negligible for 2D structures out of the strike coordinate system or 3D anomalies (compare principal axes definition in Siemon (1997)).

The strike directions in Figure 4.7 support the findings from the observations of the perturbation tensor elements themselves: Negligible effects in the western part of the array and strong 2D strike angles following the Carpentaria conductor as marked by the grey line in the Figure. The period dependence of the strike angles 


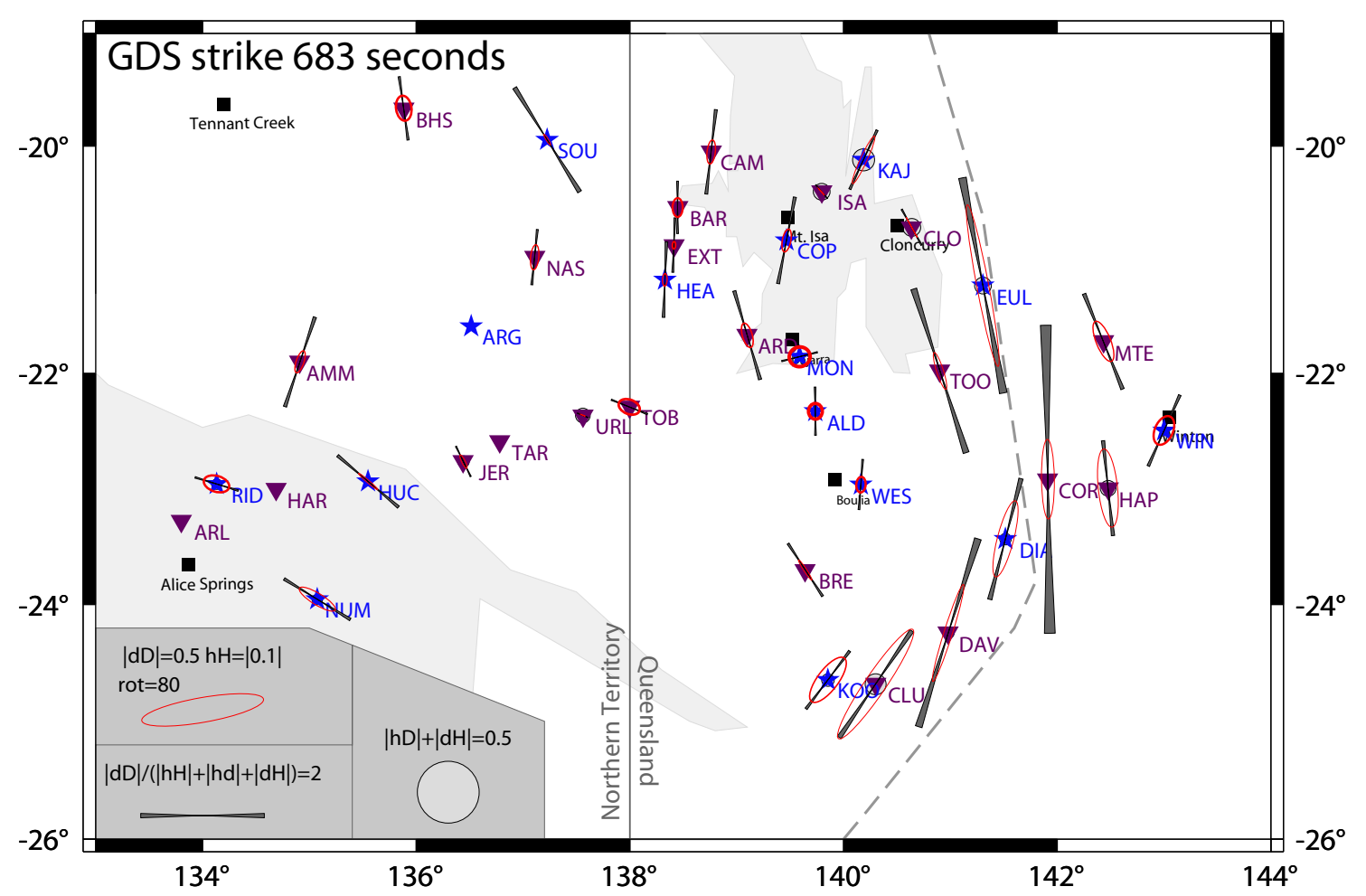

Figure 4.7: GDS strike angles for a period of 683 seconds. Strike angles were obtained by maximization of the $d_{D}$ element with respect to the other horizontal perturbation tensor elements. Ellipses indicate the relation of $d_{D}$ and $h_{H}$ while the length scales with the ratio of $d_{D}$ and the other elements. Grey circles size with the summed amplitudes of the off-diagonal elements and thus indicate three dimensional structures.

is the same as for $d_{D}$ with strongest ellipticities around 512-683 seconds.

The MT strike angles in Section 4.1.1 show spatially consistent patterns while high skew values, especially in the eastern part of the array indicate $3 \mathrm{D}$ effects. The GDS strike angles on the other hand reveal a clear strike direction along the Carpentaria anomaly and are therefore in agreement with the induction arrow observations (Section 4.1.2). This observation is related to the different natures of magnetic and magnetotelluric transfer functions. While the magnetic field is primarily sensitive to the continuous bandlike Carpentaria anomaly, the electric field reacts on small scale deviations from a straight band. This leads to three dimensional effects in the MT structures while the GDS results indicate the predominant conductive band. Therefore the MT transfer functions provide a better resolution of the local structures of the anomaly while the GDS results identify the dominant regional structures. As the large site spacing of this study does not allow a small scale resolution of the local structure of the Carpentaria conductor, the magnetic transfer functions provide good measures for regional scale investigations. In the following Chapter magnetic and magnetotelluric transfer functions will be combined to get a better insight in differences and common features of the datasets. 


\section{Combination of magnetic and magnetotelluric transfer functions}

Magnetic and magnetotelluric transfer functions are based on electromagnetic induction phenomena and are therefore sensitive to conductivity structures within the Earth. Due to the distinct nature of electric and magnetic fields however there are principal differences between the two methods. The MT method allows the determination of the conductance, at least for 1D structures (absolute conductivity for homogenous subsurface). In more complex situations electric charges can accumulate at resistivity interfaces and lead to distorted electric fields (Chapter 2.3.3) that bias the estimated apparent resistivities. Magnetic transfer functions on the other hand are sensitive to conductivity gradients only (the magnetic field measured above homogenous or layered substrata will always have twice the amplitude of the source field). Solely in the vicinity of conductivity gradients increased or reduced current densities in the subsurface lead to deviations from the 1D background field and therefore to anomalous magnetic fields at the surface (Chapter 2.2, Figure 2.2). The magnetic field is unaffected by electric distortion unless the charges accumulate at inductive depth. Only in situations where the spatial data coverage is insufficient to resolve localized conductivity anomalies the introduction of magnetic distortion is sensible. This however is closely related to anomalous magnetic fields that can be described in terms of the GDS perturbation tensor (Chapter 2.4).

These considerations illustrate that magnetic and magnetotelluric transfer functions show different sensitivities to certain structures. Therefore a combination of both methods should lead to better constraints for subsurface conductivity structures.

Becken \& Pedersen (2003) present an algorithm to compute horizontal magnetic and magnetotelluric transfer functions from local induction arrows (tipper) provided that a sufficiently dense spatial coverage is available. The method is described in some detail in Section 2.5 and can be used to investigate inconsistencies between the datasets, identify differences in MT and GDS transfer functions and calculate otherwise unavailable transfer functions from measured ones. Finally static shift effects can be removed from measured MT transfer functions. When vertical and horizontal magnetic transfer functions and magnetotelluric results are available, the converted results can be compared to the measured data. As magnetic transfer functions are of TE-mode only (Section 2.5) the method is particularly useful to separate TE- and TM-mode effects in magnetotelluric data. TE and TM mode show different sensitivities for all possible kinds of anomalies (Berdichevsky et al., 1998). Therefore the difference between measured impedance tensor and that derived from 
magnetic transfer functions provides additional information for the interpretation.

In the following the conversion algorithm is first applied to data from a 3D modeling code. Afterwards it is utilized on real data from central Australia and Germany.

\subsection{Magnetic to magnetotelluric conversions for noise free 3D model data}

In order to test the conversion algorithm a model study was performed. To incorporate $2 \mathrm{D}$ and $3 \mathrm{D}$ effects a $\pi$-shaped conductive structure of $10 \Omega m$ that extends from the surface to infinite depth in a $100 \Omega m$ host medium was modeled using the 3D forward modeling code of Mackie \& Booker (1999) (Figure 5.1). A period of 8 seconds (skin depth of $4.5 \mathrm{~km}$ in the conductor and $14 \mathrm{~km}$ in the host medium) and a site spacing of 700 meters in the central part of the model have been chosen. This good spatial coverage provides almost perfect conditions for the conversion. In a first step the anomalous horizontal magnetic field and thus the horizontal perturbation tensor elements are estimated from the local induction arrows using the iterative approach described in Chapter 2.5. These results are shown in the second row of Figure 5.1. A clear $h_{H}$-anomaly associated with the top bar is the dominating feature in both, the modeled magnetic transfer functions (1st row in Figure 5.1) and those converted from the modeled local induction arrows (2nd row). The diagonal blades of the $\pi$ lead to anomalies in all components. This effect is related to the chosen coordinate system. The structures would lead to anomalies in only one of the main diagonal elements in strike coordinates of the diagonal section of the model. The good agreement between the modeled and converted results reflect the fact that the information content in both, vertical and horizontal magnetic transfer functions is the same.

\subsubsection{Conversion of magnetic to magnetotelluric transfer functions}

In a second step the normal electric field (that related to the background structure of $100 \Omega m$ ) can be fixed by a single impedance value at a site far from the anomaly. Then the anomalous local electric fields can be estimated from the vertical magnetic field using equation (2.58). The combination of normal and anomalous electric fields leads to the local electric field values that in turn can be used to compute the MT impedances. As the magnetic field at the surface is related to horizontal currents (TE mode, Section 2.5) only TE mode electric fields can be estimated from magnetic transfer functions. For 2D and 3D structures that are associated with TM mode electric fields this leads to severe differences between measured and converted MT transfer functions.

Apparent resistivities and phases from the 3D model (3rd row) and those converted from the local induction arrows (4th row) can be found in Figure 5.1. The fact that magnetic transfer functions are TE-mode only leads to TE mode converted 


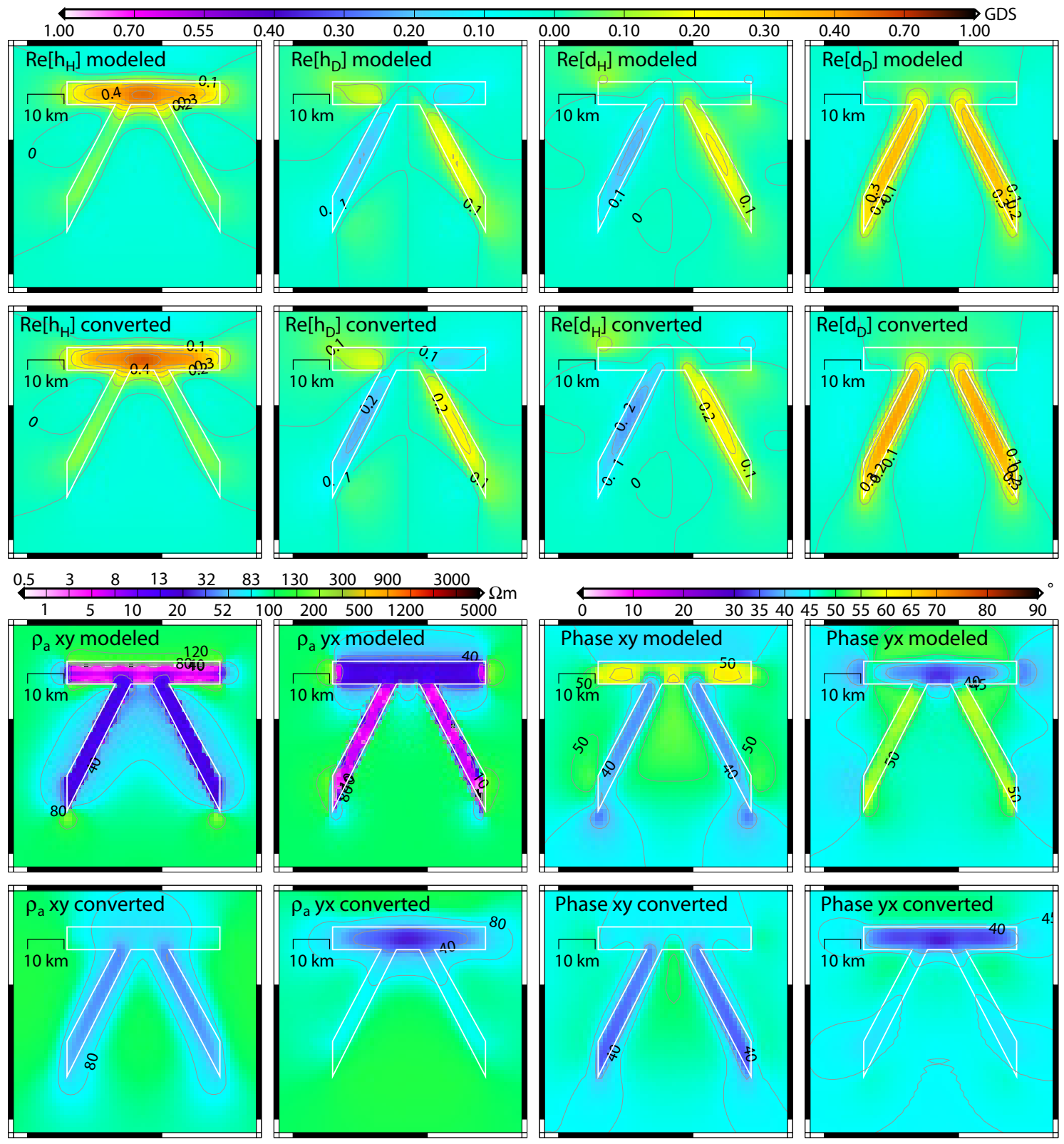

Figure 5.1: Real parts of the GDS transfer functions, apparent resistivities and phases for a $\pi$-shaped model and a period of 8 seconds. The model consists of an $\pi$-shaped conductive structure (10 $\Omega \mathrm{m}$, white outlines) that extends from the surface to infinite depth in a $100 \Omega m$ background medium. Direct results of the model code and results converted from the local magnetic transfer functions are shown.

electric fields while the MT transfer functions from the 3D model contain TE and TM mode electric fields. This is particularly obvious in the apparent resistivities where distortion effects are clearly visible in the modeled results while they are ab- 
sent in the converted results. As galvanic distortion effects do not affect the magnetic field this is a direct consequence of their TM-mode origin. The $\pi$ 's top bar strikes east-west and is long compared to the skin depth in both, the conductor $(4.5 \mathrm{~km})$ and the host medium $(14 \mathrm{~km})$. Away from the ends it can therefore be regarded as a 2D structure with the yx-component as the E-polarization of the top of the bar. Therefore the purely TE-mode converted results match the modeled apparent resistivities and phases best for the yx-component. In contrary the top bar is unresolved in the converted B-polarization (xy-component) magnetotelluric transfer functions. This is a result of the decoupling of the modes when the data is in strike coordinates of a 2D structure (Section 2.3.2) .

For elongated structures that are not visualized in strike coordinates the energy of the TE mode is distributed over all impedance tensor elements (see blades of the $\pi$ ). In that situation it is helpful to use a rotational invariant expression for the illustration. In the present situation the determinant of the impedance tensor provides a good rotational invariant measure. If it is normalized by the background impedance it reproduces the correct apparent resistivities of the induction space. Different normalizations of the determinant can be used to identify strong telluric distortions (D. Rippe, pers. communication). Figure 5.2 shows apparent resistivities and phases of the normalized determinant of the modeled and converted impedance tensor. The converted apparent resistivities clearly show the $\pi$-shaped structure reaching the modeled value of $10 \Omega m$ above the conductor where the modeled results are dominated by distortion effects. As galvanic distortion is not present in the converted results these are easier to interpret. The phases of the determinant show large differences between converted and modeled results. As expected for TE-mode (E-polarization) the converted phases above the conductor are decreased, indicating decreasing conductivity with depth or lateral distance. The modeled phases however are mostly increased as they would in B-polarization of a 2D anomaly. Therefore they are dominated by TM-mode electric fields. This was already evident in the phases of the impedance tensor in Figure 5.1.

In conclusion the model study of the $\pi$-shaped model illustrates the applicability of the algorithm. The horizontal magnetic transfer functions could be estimated to about the same values as those obtained by the direct modeling. The restriction of the converted transfer functions to TE-mode results leads to unresolved structures in the B-polarization. This restriction however allows the removal of static shift effects on the data as these are purely of TM-mode origin. The conversion algorithm uses all components of the vertical or horizontal magnetic transfer functions leading to the estimation of the entire TE-mode impedance tensor. Therefore the conversion results are coordinate system independent. For $2 \mathrm{D}$ structures the full information of the conversion can be illustrated by plotting the MT impedances in the strike coordinate system. The corresponding results are (apart from differences in the definition of the regular input grid) equal no matter if the rotation is performed prior or after the conversion. In case of multidimensional structures rotational invariants can be used to visualize 3D effects. 


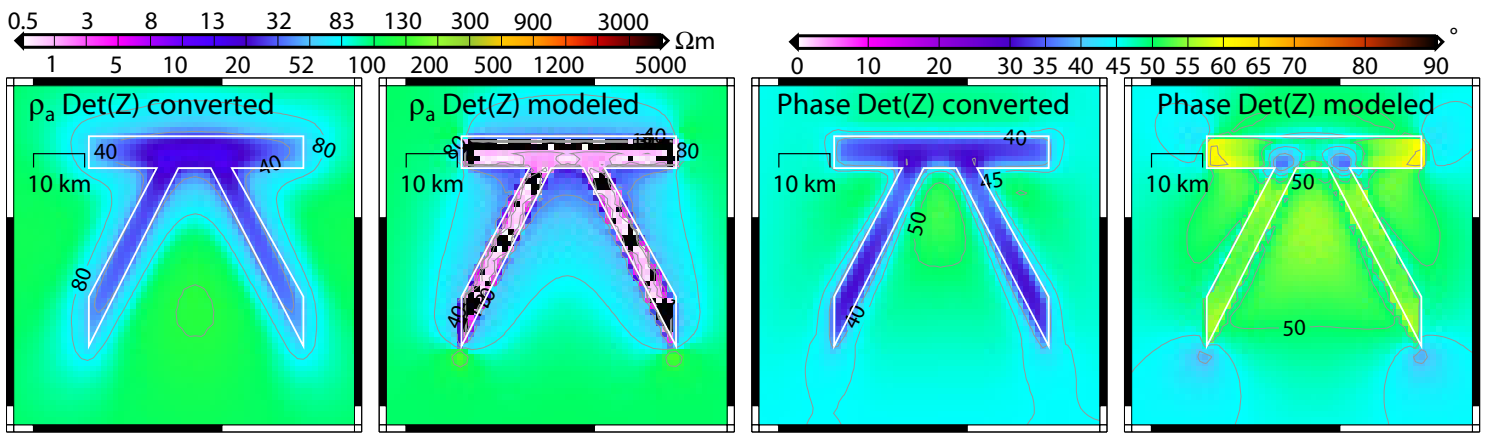

Figure 5.2: Apparent resistivities and phases of the normalized determinant Det $=\left(Z_{x x} Z_{y y}-Z_{x y} Z_{y x}\right) / Z_{1 D}$ of the impedance tensor for the $\pi$-shaped model. Results from the 3D model and those converted from the local induction arrows are plotted.

\subsubsection{Multidimensional boundaries}

In the previous example the background structure was 1D. Independent of the structures in the center of the model this implies vanishing average vertical magnetic fields at the model borders. This changes when $2 \mathrm{D}$ boundaries exists. In that case the algorithm leads to biased results. This is related to different normal electric fields on both sides of a 2D conductivity contrast. In the wave number domain approach the normal part of the vertical magnetic field is assumed to vanish $\left(B_{z}(k=0)=0\right)$. For multidimensional boundaries this is not true and additional terms in equation (2.58) have to be introduced. These however lead to an underdetermined problem that has no general solution. In some circumstances, e.g. known 2D structures it is nevertheless possible to estimate correct local electric fields from the vertical magnetic field if this is performed in the space domain. For a strike in x-direction directions the z-component of equation (2.57), $\frac{\partial E_{y}}{\partial x}-\frac{\partial E_{x}}{\partial y}=-i \omega B_{z}$, simplifies to $\frac{\partial E_{x}}{\partial y}=i \omega B_{z}$ and the anomalous electric field (with respect to the reference field at one side of the boundary) can be estimated by a simple 1D integration

$$
E_{x}^{a}(y)=-i \omega \int_{0}^{y} B_{z}\left(y^{\prime}\right) \mathrm{dy}
$$

Adding the normal field to the estimated anomalous field leads to correct local field values. If the regional structure is known to be that of quaterspaces, the normal field far from the boundary is $1 \mathrm{D}$ itself and the this normal field can be used to fix the electric field in the perpendicular polarization (Appendix C). For unknown 2D or multidimensional boundary structures however it is not possible to estimate the correct local fields with the given algorithms as the horizontal electric field gradients are inseparably linked to the vertical magnetic field. 


\subsection{GDS to MT conversions of the Australian dataset}

The Australian dataset presented in Chapter 4 shows laterally quite consistent results. When MT and GDS methods are compared however some differences in the signatures become evident. Especially the induction arrows in the western part of the array appear large in comparison to the effects seen in the magnetotelluric and horizontal magnetic (GDS) transfer functions. The conversion algorithm provides the opportunity to check for possible inconsistencies. Furthermore the large but spatially even site spacing provides good conditions for the application of the conversion algorithm.

\subsubsection{From tipper to horizontal magnetic transfer functions}

While the model data of the previous section was calculated for an even site spacing the dataset from Australia has to be prepared for the conversion. The following steps have to be performed:

- creation of uniformly spaced input data. This is done by spatial interpolation using the program surface of the GMT package (Wessel \& Smith, 1998). Note that the grid cell size used for the interpolation influences the results of the algorithm as small cells lead to smoother variations between neighboring sites. Here a cell size of $0.25 \times 0.25$ degrees, an interpolation area from 131 to $146^{\circ}$ longitude and -27 to $-18^{\circ}$ latitude, a surface tension of $\mathrm{T}=0.3$ (see surface manpage) and interpolated amplitudes limited to the $\mathrm{min} / \mathrm{max}$ values at measured sites were used.

- application of a window function (Hanning window) prior to the Fourier transformation to improve the spectral resolution of the non-periodic input data. In order to prevent measured data points from the down biasing of the window function the survey area was extended by extrapolation.

A transformation of vertical to horizontal magnetic transfer functions is only sensible when neighboring sites show consistent behavior. Therefore the large site spacing of the dataset allows a transformation for long periods ( $>1000$ seconds) only. Figure 5.3a shows the measured local real/imaginary induction arrows (red) for a period of 1024 seconds that was used for the transformation. Figure 5.3b shows the measured real part of the $d_{D}$ perturbation tensor element while Figure $5.3 \mathrm{c}$ shows the $d_{D}$ element that was approximated from the tipper values with the program tipper2mt (see Appendix B.2.4). In general a good agreement between the measured and converted response function can be seen. Only in the south-eastern corner differences are visible. These are related to a bend in the conductive Carpentaria anomaly toward the west leading to a reversal in the induction arrow direction above the anomaly that is not resolved by the present dataset, but by the dataset of Chamalaun \& Barton (1993). Therefore the extrapolation leads to north-westerly instead of south-easterly pointing arrows due to missing data coverage in that area. 


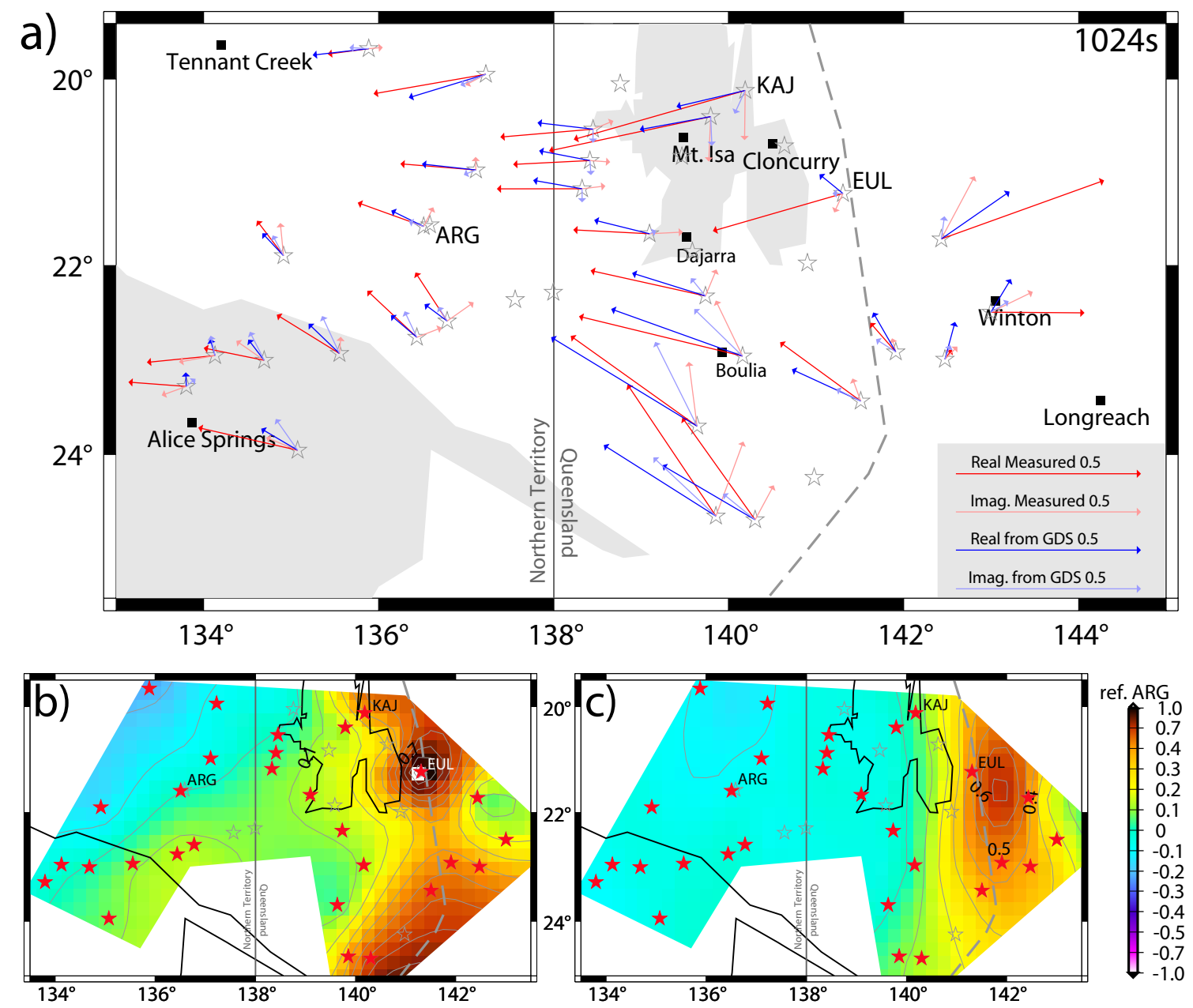

Figure 5.3: (a) Local induction arrows for a period of 1024 seconds as measured (red) and converted from the horizontal GDS transfer functions (blue). (b) Real part of the perturbation tensor element $d_{D}$ for a period of 1024 seconds as measured and (c) as estimated from the local vertical magnetic transfer functions. The red stars in (b) $+(\mathrm{c})$ indicate the field sites used for the plot respectively the conversion.

Another distinct feature is the significantly reduced maximum amplitude at site EUL. Next to effects that are related to the interpolation process these could be related to the complicated 3D structure at that site. The other estimated (not shown) and measured (Appendix A.3) perturbation tensor elements show primarily increased values in the south-eastern part only. This is related to $3 \mathrm{D}$ effects associated with the change in direction of the Carpentaria conductivity anomaly towards the west and is indicated in Figure 5.3a by imaginary induction arrows that are not in line with the real arrows as they would for 2D structures.

In addition to the measured local induction arrows Figure 5.3a plots induction arrows that were converted from the measured horizontal perturbation tensor el- 
ements (blue). Largely converted and measured induction arrows agree well with each other. Only in the western part where the amplitudes are small the agreement is limited. This is related to the selection of the GDS reference site. While ideally the reference site's measured horizontal GDS transfer function vanish, local induction arrows exist at site ARG, leading to the observed differences. Generally smaller converted than measured induction arrows can be attributed to the different behavior of horizontal and vertical magnetic fields. While the anomalous horizontal field and thus the horizontal GDS transfer functions vary smoothly over conductivity gradients the vertical field varies far more rapidly and changes sign above a horizontal interface (compare Figure 2.2c). The interpolation of the horizontal transfer functions amplifies this effect by additional, grid cell size dependent smoothing and leads to smaller converted induction arrows.

\subsubsection{Conversion of magnetic to magnetotelluric transfer functions}

Following the dimensionality investigations in Chapter 4 site ARG (with an app. resistivity of $400 \Omega m$ ) was chosen as electric field reference for the Australian dataset. As local induction arrows and GDS transfer functions are available it is possible to estimate MT impedances from both types of magnetic transfer functions. Figure 5.4 compares the measured MT apparent resistivities (with distortion) and phases of the off diagonal impedance tensor elements and a period of 1024s with those estimated from the local induction arrows (tipper) and the GDS transfer functions. Assuming the north-south striking Carpentaria anomaly as the dominant 2D structure the xy-component is the E-polarization and the yx-component the B-polarization. The measured apparent resistivities are dominated by the distortion effects in the data. The E-polarization results in the eastern part however show undistorted, low resistivities associated with the sediments and the Carpentaria anomaly in that area (compare Section 4.1). This is reproduced in the E-polarization apparent resistivities converted from the magnetic transfer functions while the anomaly is obviously unresolved by the B-polarization. The absolute values of the apparent resistivities however are higher than the mostly undistorted measured resistivities in the east. Such an effect is produced by a regional 2D conductivity contrast, i.e. higher resistivities in the west and lower resistivities in the east. In that case the mean value of the vertical magnetic transfer functions does not vanish and equation (2.58) is no longer valid. As described in Section Section 5.1.2 this problem can be overcome for $2 \mathrm{D}$ structures by integration in the space domain but due to insufficient data coverage east of the Carpentaria anomaly and 3D effects in the data, a simple 2D assumption does not lead to convincing results in the present case. Consequently fixing the electric field in the western part (site ARG) leads to higher than real converted MT impedances and thus apparent resistivities in the east (compare Appendix C). Although sparsely sampled in the east, the induction arrows in Figure 5.3 might support higher conductivities east of the Carpentaria anomaly (positive integrated $z_{D}$ component in East-West direction). Such a regional contrast could 

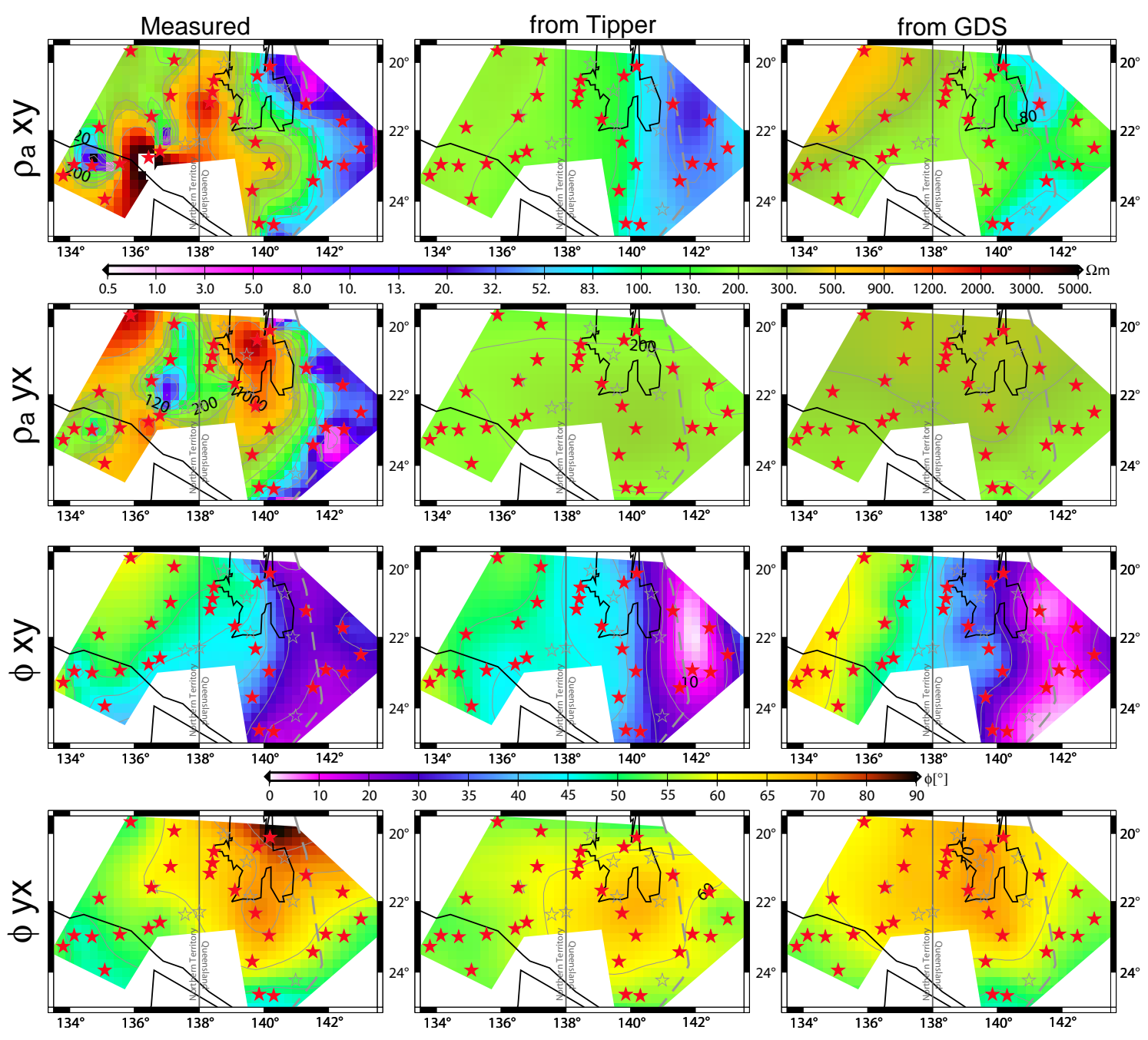

Figure 5.4: Apparent resistivities and phases of the off-diagonal impedance tensor elements as measured and as converted from induction vectors and GDS transfer functions for a period of 1024 seconds.

be explained by the boundary between the Proterozoic Mt Isa block and younger eastern Australia supporting the hypothesis that the Tasman Line, the boundary between the old western and the younger eastern part of Australia (Chapter 3), actually follows the Carpentaria conductivity anomaly instead of running further east. This idea will be discussed in Chapter 6 .

The measured and converted phase values agree well for the $\mathrm{xy}-$ and $\mathrm{yx}-$ polarization. As the converted results contain TE-mode information only this implies that the measured MT phases at long periods are dominated by TE-mode fields. This is in agreement with model studies that show that TM-mode fields are sensitive to near surface structures while the TE mode is more sensitive to deep structures (Berdichevsky et al., 1998). Therefore conversions of magnetic to magnetotelluric transfer functions are in better agreement to measured data for long periods. Better 
fits for the MT phases than for the apparent resistivities follows their sensitivity to resistivity changes rather than absolute values making them unaffected by different normal electric fields away from conductivity contrasts.

\subsection{Application to MT data from Germany}

The promising conversion results from Australia encourage the application of the conversion algorithm on other datasets. A large dataset of long period MT data for Germany has been measured by Moorkamp (2003), Gatzemeier (2002), Leibecker (2000) and others and has been interpreted in terms of anisotropic structures in the upper mantle below Germany (Leibecker et al., 2002; Gatzemeier \& Moorkamp, 2005; Moorkamp \& Gatzemeier, 2006). Due to the dense site spacing and the mostly good data quality the dataset is well suited for the conversion approach. Due to the focus on the methodology this study will focus on the conversion results from the German dataset rather than their implications for regional conductivity structures.

Figure 5.5 plots measured apparent resistivities, phases and local induction arrows for a period of 1024 seconds in a geomagnetic coordinate system. The apparent resistivities show strong distortion effects in wide parts of the array. Only the low resistivities in the northern part (north German sediments) seem to be largely unaffected by distortion effects. The phases show moderate lateral variations with strong phase differences between the two polarizations in the center of the array. Due to the less than ideal site spacing in the border areas, some structures are only seen by one site and are therefore not well resolved. While Leibecker (2000) investigated GDS results for the Eifel region no GDS transfer functions for the whole array have been estimated. These could be obtained with a geomagnetic observatory as common reference but their calculation is beyond the scope of this study. Therefore the estimated GDS results cannot be compared to measured ones.

The local induction arrows in Figure 5.5 show different regions of common arrow directions. Especially in the center of the array a clear change in direction can be observed. This change is associated with three dimensional effects indicated by angles around $90^{\circ}$ between real and imaginary arrows.

The conversion requires an electric field reference in terms of a $1 \mathrm{D}$ impedance at one site. Due to only minor multidimensional effects in the data and the small induction arrows site SPRS (with apparent resistivity of $9 \Omega m$ ) in the northern part of the array was chosen as reference. Figure 5.6 shows the conversion results of the real parts of the horizontal GDS perturbation tensor elements and the MT phases. Induction arrow data from all sites marked by stars have been used for the estimation. The chosen lateral discretization is indicated by the block sizes in the Figure. While the spatial resolution is good for the central part of the array some peripheral areas are not well covered.

The estimated GDS results reflect the complexity of the dataset. The north German sediments are clearly associated with a positive $h_{H}$ anomaly. In the center of the array a strong $d_{D}$ anomaly is visible. As this anomaly is associated with signa- 
tures in the other transfer functions it is created by a buckled 2D bandlike structure striking north-east or 3D structures. The elongated structures inferred from the GDS results match with the conductive structures used by Moorkamp \& Gatzemeier (2006) to model upper mantle anisotropy. As infinitely extended anisotropic structures are not associated with GDS anomalies this implies that laterally confined structures (anisotropic or not) have to be the origin of the estimated anomalies.

The converted MT phases (Figure 5.6) reflect the major structures that were evident in the measured results. In particular the yx-polarization matches quite well (apart from the high phases in the north-western corner of the measured data that is only seen by one site). In the center of the array the xy-polarization is in agreement with the measured results while the more sparsely sampled peripheral areas show stronger deviations. In general a good correlation between yx-polarized MT results and the $h_{H}$ perturbation tensor element and respectively xy-MT and $d_{D}$ GDS tensor element is evident. This behavior follows directly from the calculation of the electric field (equation 2.57).

Figures 5.7 $(\mathrm{a}+\mathrm{b})$ plot the apparent resistivities estimated from the local induction arrows. As no galvanic distortion is present in the TE mode converted electric field, the apparent resistivity shows only little spatial variations and the same structures as seen in the GDS and MT phase results are evident. While the low resistivities in the north German sediments (around site SPRS) reflect their high conductivities the resistivities obtained for the rest of the array are lower than expected from 3D modeling of the dataset (Leibecker, 2000). This is related to lower resistivity values (and therefore lower normal electric fields) in the rest of the array and possibly indicates large scale conductivity variations from south to north (compare the discussion of the Australian dataset). The complex structures in central Germany however can even less be approximated by 2D structures and no correction for multidimensional boundaries is possible.

Figure 5.7c shows measured (black) and converted (white) MT strike angles on top of the real part of the converted $d_{D}$ perturbation tensor element. The phase difference between xy- and yx-polarization is indicated by the length of the segments. The measured strike directions point in the direction of the higher phase while the converted strikes are confined in the 0-90 degree range. As single site MT strike angles suffer from a $90^{\circ}$ uncertainty, perpendicular angles might represent the same strike. A comparison of measured and converted strike directions and the corresponding phase differences shows significant differences in some areas. In particular in the central part where a change in direction of the measured results towards northerly directions is evident, large differences can be seen. This change is associated with 3D structures that are indicated by increased $d_{D}$ and non-vanishing $d_{H}, h_{D}$ and $h_{H}$ GDS perturbation tensor elements. This interpretation is supported by the GDS strike ellipses in Figure 5.7d. While the ellipses would reduce to lines in the $2 \mathrm{D}$ case, smaller ellipticities and non-vanishing $h_{D}$ and $d_{H}$ elements as in Figure 

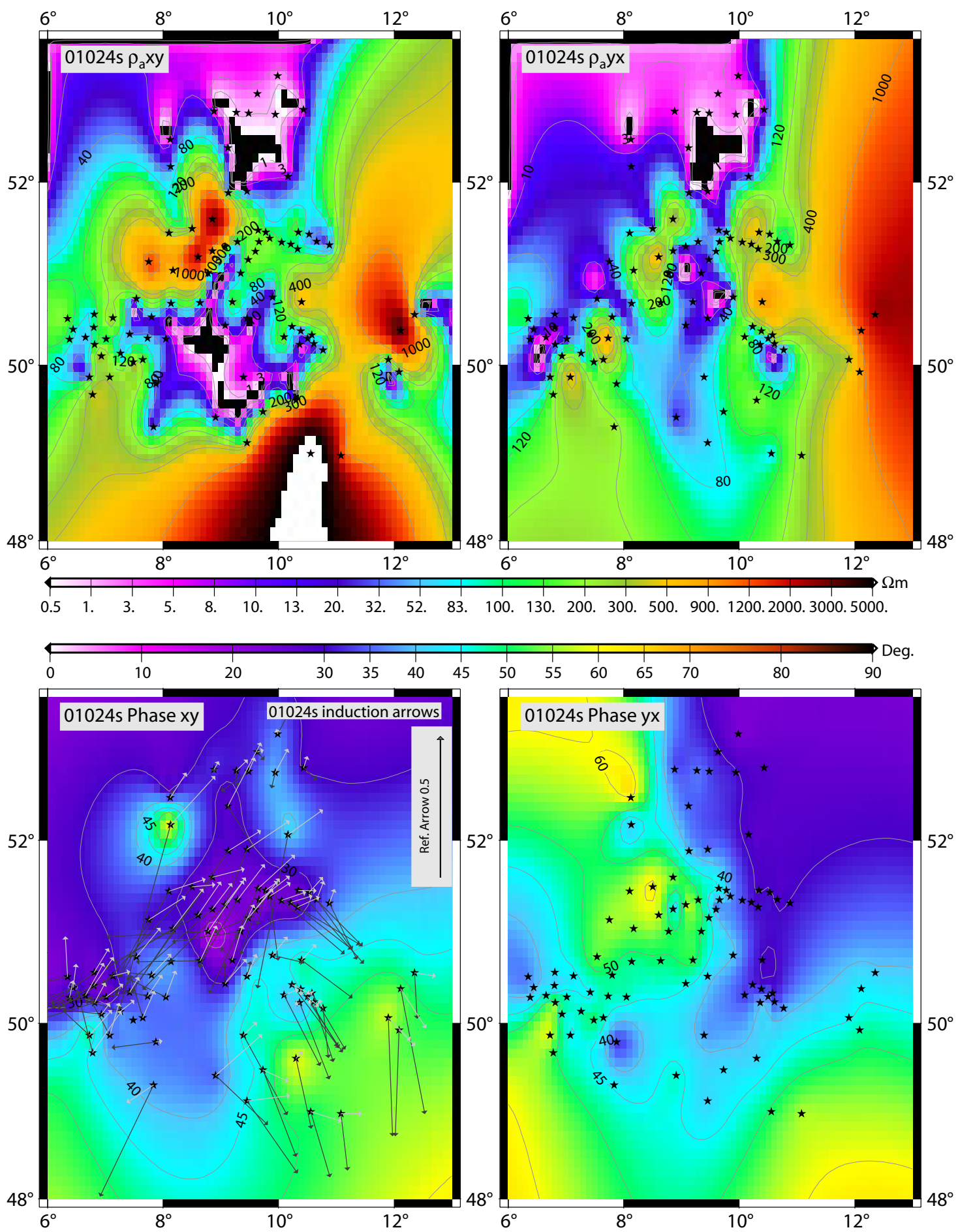

Figure 5.5: (top) Measured apparent resistivities and (bottom) phases of the offdiagonal impedance tensor elements from central Germany. Real (black) and imaginary (grey) local induction arrows are plotted in the lower left graph. 

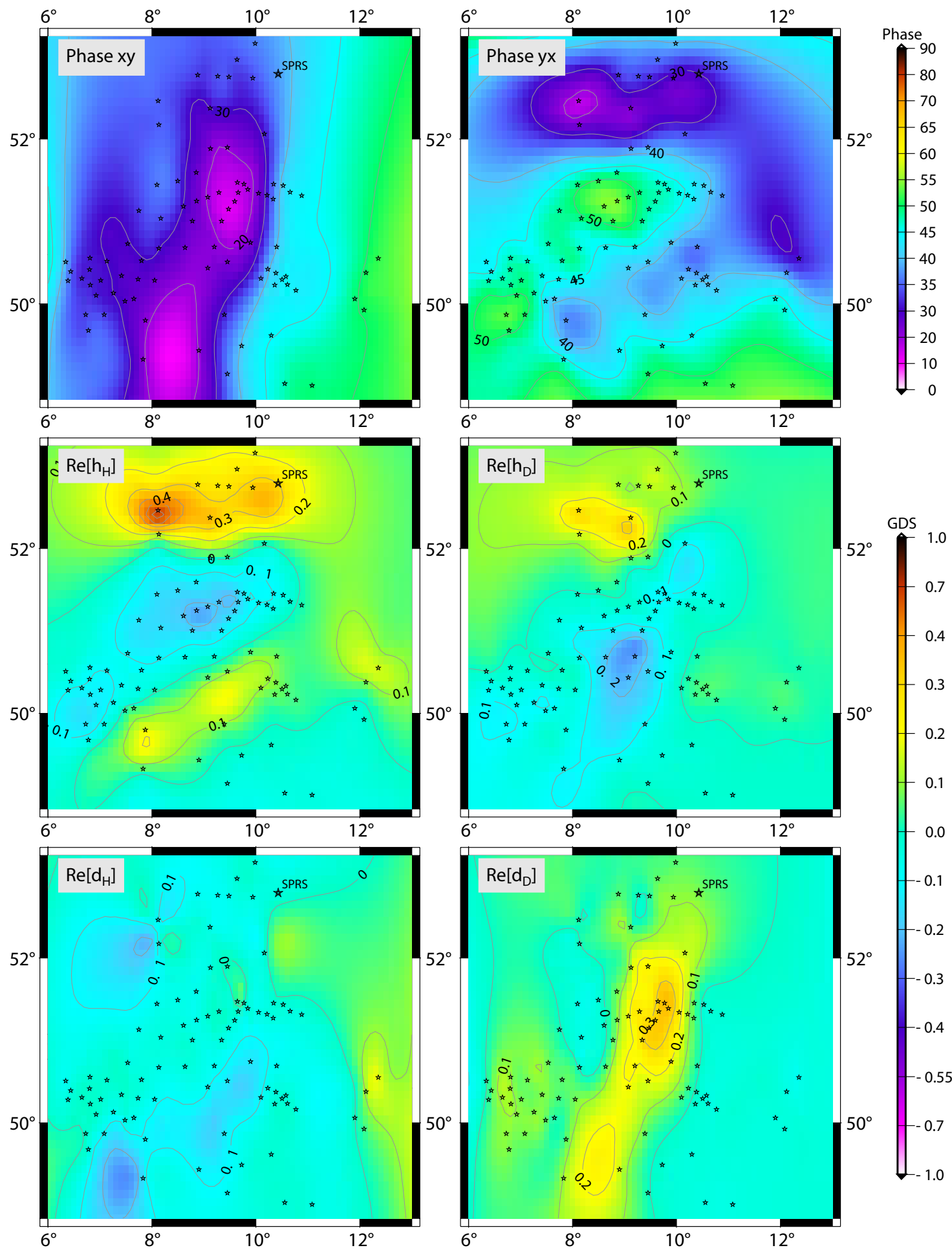

0.1

0.2

0.3

0.4

0.55

0.7

1.0

Figure 5.6: Phases of the off-diagonal impedance tensor elements and real parts of the horizontal GDS perturbation tensor elements in central Germany as converted from the local induction arrows at a period of 1024s. The impedance at site SPRS in northern Germany was used as electric field reference. 

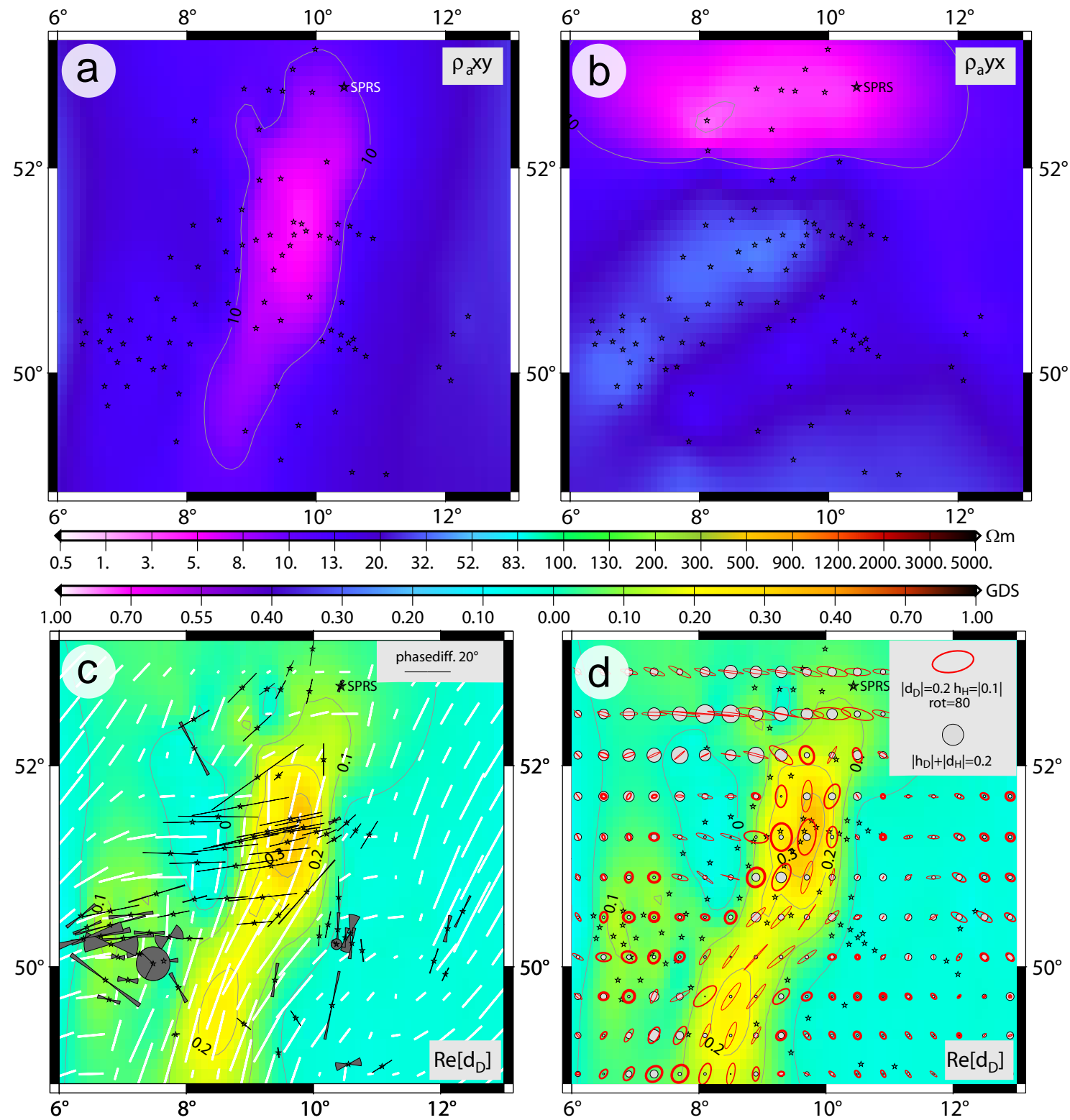

Figure 5.7: $(a+b)$ Apparent resistivities at 1024s in central Germany as converted from the local induction arrows. Impedances at site SPRS have been used to estimate the local electric field. (c) Measured (black) and estimated (white) MT strike angles. The length of the wedges indicates the phase difference between $\mathrm{xy}$ and yx polarization in strike coordinates. (d) GDS strike ellipses and off-diagonal element magnitudes of the converted GDS tensor. The strike direction was obtained by a minimization of $d_{H}, h_{D}$ and $h_{H}$ with respect to $d_{D}$. The major axes illustrates the amplitude of $d_{D}$ in strike coordinates while the minor axes scales with $h_{H}$. The background in $(\mathrm{c}+\mathrm{d})$ shows the real part of the estimated $d_{D}$ perturbation tensor element. 
5.7d indicate 3D structures. As multi-dimensional structures are associated with TM-mode electric fields the conversion algorithm that is based on the TE mode magnetic field is of limited use to reproduce the measured results. This might as well be the reason for the moderate agreement between measured and converted phases in Figure 5.6.

\subsection{Guidelines for tipper to GDS/MT conversions}

The application of the conversion algorithm on modeled data and the real datasets from Australia and Germany gave a good impression of the capabilities and limitations of the method. To obtain good results several precautions have to be followed. These, the potential benefits of the conversion and the limitations of the method are summarized in the following.

\section{Requirements and precautions}

- 3D array data with good spatial coverage

- site spacings that are smaller than the skin depth of the investigated period in the most conductive regions

- preferably uniform background structures. For moderate regional differences the conversion might still lead to qualitatively correct results while the apparent resistivities will be biased. For known 2D structures the regional conductivity differences can be recovered.

\section{Benefits}

- chance to obtain transfer functions that are otherwise not available

- consistency check for different datasets, e.g. magnetic and magnetotelluric transfer functions

- possibility to remove static shift effects from MT data (only for uniform background structures). Note that for inversion or forward modeling purposes a removal of electric distortion created by resolvable structures (possibly leading to static shifts for the longest periods only) is not desired as it is part of the modeling parameters.

- possibility to separate TE and TM mode effects in the data

\section{Limitations}

- magnetic transfer functions are solely of TE mode origin. Therefore converted MT results are constricted to TE mode 
- for complicated 3D conductivity structures the limitation to TE mode leads to strong differences between measured and converted MT results. This affects $2 \mathrm{D}$ strike directions and other measures as well.

- interpolations to obtain a regular grid for the Fourier transformation leads to spatial smoothing of the results. Therefore the converted results will always be smoother than the measured ones. This is particularly important when induction arrows are converted from horizontal GDS transfer functions.

- The algorithm cannot be used if unknown 2D or 3D resistivity contrasts are present at the array boundaries. This is related to the negligence of the constant component of the vertical magnetic field in equation (2.58).

Differences between the converted and measured results do not necessarily indicate inconsistencies in the datasets but can be related to TM-mode effects in the measured data. In that case the differences can be used to simplify the investigation of TM-mode effects. For modeling purposes of the data this provides additional constraints for the final model. The influence of TM-mode effects is strongest for shallow structures and diminishes when the size of the structure becomes small compared to the induction space (Berdichevsky, 1999; Berdichevsky et al., 1998).

In relation to the local induction arrows the horizontal GDS transfer functions are usually better suited for the conversion to MT impedances. This is related to their smoother variations above conductivity contrasts.

Note that the transformation results are independent of the coordinate system when the full MT and GDS tensors are considered. Backward rotation of the converted transfer functions into the original coordinate system leads to the same results as a direct transformation of unrotated induction arrows. Slight difference might arise from parameterization effects during the creation of the regular input grid. Due to the interpolation of the magnetic transfer functions a proper error propagation for the resulting magnetotelluric transfer functions is difficult. In any case large errors in the vertical magnetic transfer functions will lead to poorly defined converted transfer functions. 


\section{3D Modeling of the data}

In most situations the major structures in MT and GDS data can already be inferred directly from the transfer functions. This is in particularly true for undistorted 1D structures. In more complex environments direct interpretations are complicated by the inductive nature of electromagnetic depth sounding techniques as perioddependent transfer functions are affected by laterally and vertically removed structures. In order to distinguish between vertically and laterally removed formations multiple measuring sites are required. Computer modeling codes can help to resolve the conductivity structure in the Earth. This can be done by either forward modeling or inversion. In forward modeling studies an initial resistivity model is created and refined in various steps to fit the data. Inversion schemes use mathematical algorithms for model alterations. A general problem with both approaches is the under-determined nature of the data. In forward modeling usually least structure models are developed to avoid the inclusion of unresolved structures while inversion schemes usually apply regularizations, i.e. restrictions on the roughness of the model. Both approaches have been used in the present study and are presented in the following. Afterwards I discuss structural implications for central Australia.

\subsection{D forward modeling}

3D forward modeling codes allow the estimation of the electromagnetic response functions of a given resistivity model. The input resistivity model is given in parameterized form, usually rectangular blocks of a certain resistivity. For MT and GDS studies usually homogenous source fields are used. From these the modeling code estimates the electric and magnetic fields in each model cell or through the cell boundaries. Of primary interest for MT and GDS studies are the field values at the surface of the model as these can be used to estimate the MT and GDS transfer functions at each surface cell of the model.

The model simulations of this study are performed with the finite-differences algorithm of Mackie \& Booker (1999). This algorithm was modified to compute MT, and GDS transfer functions. The input model consists of cuboid model cells with homogenous resistivities within the cells. The model is illuminated by a plane wave source from above and the local field amplitudes at the surface of each block are computed in an iterative approach (conjugate gradient method, Mackie \& Madden (1993); Mackie et al. (1994)). Good convergence of the code requires moderate field gradients within the model cells. Therefore several gridding rules have to be followed (Mackie \& Booker, 1999). Highly overparameterized problems with significantly 
more model parameters than available data values are the outcome. This requires special attention to the significance of structures in the model.

The development of a 3D forward model is usually performed in several stages. In a first step the major structures of the model are defined. This can usually be done from direct investigations of the electromagnetic transfer functions as in Chapter 4. MT impedance tensor amplitudes are often affected by galvanic distortion (Chapter 2.3.3). Accordingly interpretations of undistorted measures like the phases of the impedance tensor and the GDS transfer functions are often preferable if magnetic distortion is absent. As these measures are sensitive to variations in conductivity only, a background model (e.g. inferred from 1D inversions) that defines the absolute resistivity values is required.

In a second step the significance of the previously defined major structures in the model response are checked by successive alteration of their parameters (resistivities, thicknesses, widths). Bodies of negligible or minor importance should not be included in the final model. This leads to a least structure model that prevents the final model to be visually dominated by unresolved small scale structures. Afterwards the model parameters of the remaining structures are varied systematically to find the best fitting model.

Structures defined by other geophysical methods can often be included in electromagnetic models. As theses methods investigate different physical parameters (e.g. density, acoustic wave speed or remanent magnetization) they do not have to be similarly sensitive to a given structure (Gallardo \& Meju, 1993). Major structural boundaries however often show distinct signatures in numerous physical parameters and their location in electromagnetic models can therefore be refined by the combination of different methods.

\subsubsection{D model of the Australian dataset}

According to the discussion of the MT and GDS transfer functions in Chapter 4 the most prominent features in the Australian dataset are (i) the resistive Mt Isa Block, (ii) the highly conductive Eromanga basin in the east, (iii) the Carpentaria conductivity anomaly at the eastern boundary of the Mt Isa Block and (iv) moderate resistivities in an approximately $1 \mathrm{D}$ crustal environment in the western part of the array. The depth extent of these structures can be inferred from the period dependence of the transfer functions. According to the period dependence the Carpentaria anomaly extends throughout the crust, possibly even the whole lithosphere. The crystalline Mt Isa Block is highly resistive at shallow depths. However, high phases, particularly at periods of hundreds of seconds indicate significantly increased conductivities with depth. The conductivity of the Eromanga Basin can be determined to be about $1 \mathrm{~S} / \mathrm{m}$ by investigations of the presumably undistorted apparent resistivities in the eastern part of the array (Figure 4.1). The period dependence implies sedimentary thicknesses in the kilometer range.

From these observations a starting model for the Australian dataset was developed. Due to the high conductivity contrasts between the conductive Eromanga 
Basin and Carpentaria conductor (about $1 \mathrm{~S} / \mathrm{m}$ ) and the resistive Mt Isa Block (several $1000 \Omega \mathrm{m}$ ) the gridding rules require small block width in the central part of the model. As a compromise a block width of $6250 \mathrm{~m}$ in the central part of the array was chosen. This is much smaller than required for the measured site spacings of $50 \mathrm{~km}$. For the same reason small block thicknesses (182m for the top layers) had to be used leading to final model dimensions of $173 \times 197 \times 45(\mathrm{~N}-\mathrm{S} / \mathrm{E}-\mathrm{W} /$ vertical $)$ blocks. As the grid cell sizes are increased towards the model boundaries this leads to a total model space of more than $1510 \mathrm{~km} \times 1730 \mathrm{~km} \times 200 \mathrm{~km}$. A plot of the horizontal model grid can be found in Appendix D.

In order to refine the location of the major structures the results of other methods (Chapter 3) have been included in the model. In detail these are the extent of the Mt Isa Block below the covering sediments from gravity (Figure 3.5) and magnetic anomaly (Figure 3.6) data, the sedimentary thicknesses of the Eromanga Basin (Figure 3.1), information about the Carpentaria anomaly from previous electromagnetic soundings (Chamalaun et al., 1999; Lilley et al., 2003) and the crustal thickness from seismological investigations (Figure 3.3). Despite the focus on crustal structures the strong resistivity contrasts and subsequent significant differences in penetration depth of the electromagnetic signals require the inclusion of mantle conductivities in the model to represent the long period data correctly. Following the quiet-day magnetic transfer functions for Australia (Campbell et al., 1998) the resistivity in the deep part of the model $(>140 \mathrm{~km})$ has been fixed to $50 \Omega \mathrm{m}$. This value is significantly lower than upper mantle resistivities of other continents (Olsen, 1998) and possibly indicates higher heat flux in the area (Lilley et al., 1981).

From this a-priori information we construct a first model and vary it to fit the measured data. As the conversion of magnetic to magnetotelluric transfer functions in Chapter 5 showed convincing results for long periods, both datasets were fitted simultaneously in the modeling process. The actual fitting parameters include the MT phases, GDS perturbation tensor elements and the local induction arrows.

Figure 6.1 shows the resistivity structure of the final model. While the major structures are evident, smoothed resistivity contrasts have been used to improve the convergence of the modeling code. The model response in terms of the off-diagonal element MT phases and local induction arrows are plotted in Figure 6.2. Figure 6.3 illustrates the real parts of the model's $d_{D}$ perturbation tensor element. The corresponding measured results can be found in Chapter 4, Figure 4.2 (phases, page 48 ), Figure 4.4 (induction arrows, page 50) and Figure $4.6\left(d_{D}\right.$, page 55). Results for the other modeled perturbation tensor elements can be found in Appendix D. In order to simplify the comparison, interpolations between the model responses of field site locations rather than the full modeling results with much more model sites are plotted. The effect of this procedure on the model response plots is discussed in Appendix D.

The model best represents the measured MT phases. Only slight differences presumably related to smaller scale structures are visible. However, the modeled amplitudes of the magnetic transfer functions above the Carpentaria anomaly are significantly smaller than the measured ones, indicating insufficient conductance of 


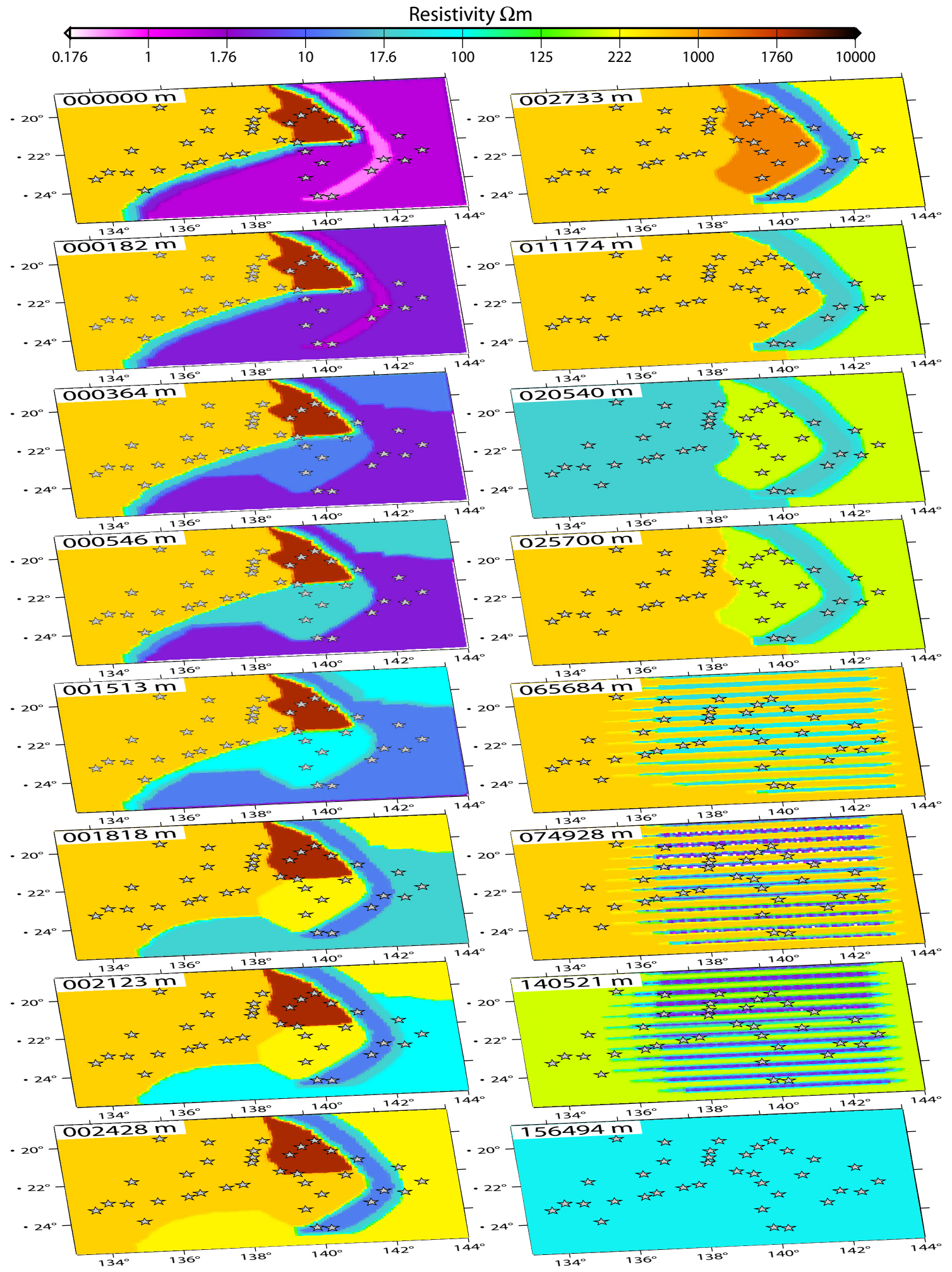

Figure 6.1: Resistivities of the different layers in the final 3D forward model for the Australian dataset. The depth of the top of each layer are plotted in the upper left corner. The resistivities in between two layers equals the resistivity of the upper layer. 


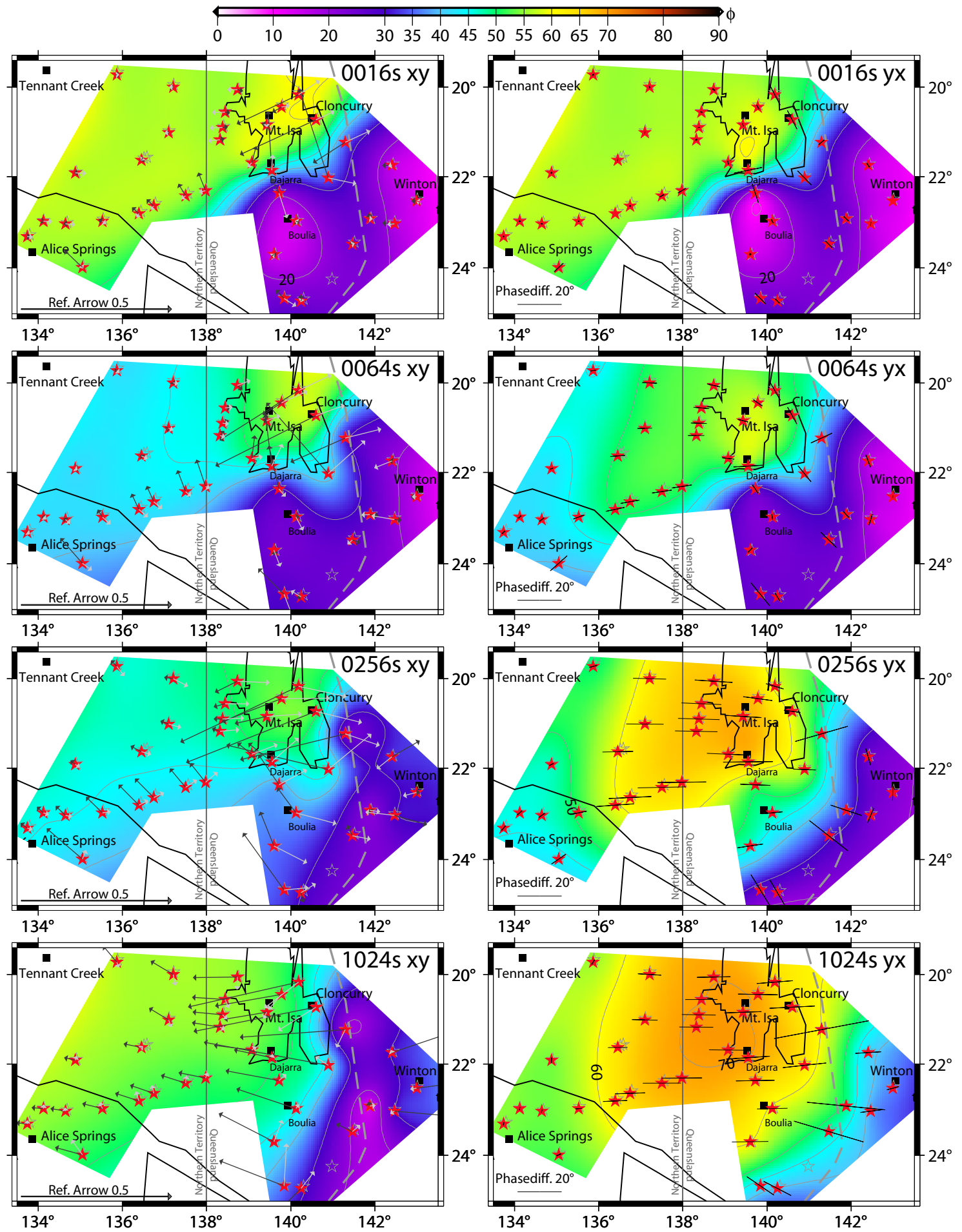

Figure 6.2: Phase responses for the final 3D forward model using spline interpolations between results at field sites for periods between 16 and 1024 seconds as well as local induction arrows (left, black=real, gray=imag) and Bahr's strike angles (right). Sites used for the interpolation are indicated by red stars. The associated measured results can be found in Figure 4.2 page 48. 


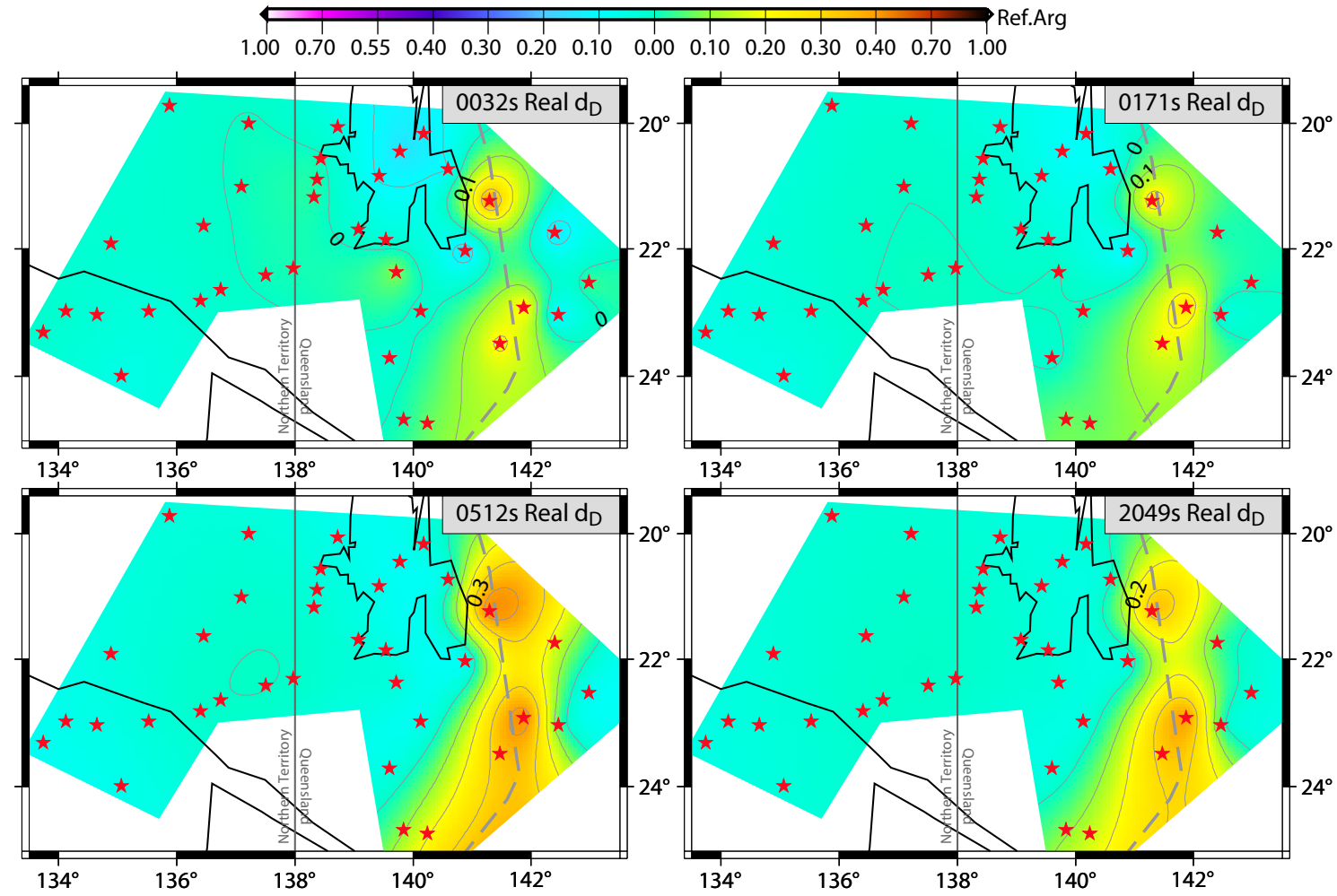

Figure 6.3: Interpolations of the real parts of the $d_{D}$ perturbation tensor elements for the final $3 \mathrm{D}$ forward model. Sites used for the interpolation are indicated by red stars.

the Carpentaria anomaly in the upper layers. Inclusion of even higher conductivities in the top layers is hindered by convergence problems for the given model gridding. Sectional modeling however allows raised conductivities leading to higher amplitude magnetic transfer functions, lower MT phases for short periods and therefore a better fit for the measured data. Consequently the upper layer conductivities of the final model are slightly too small.

In general, parameters calculated from the model response (including the perturbation tensor elements in Appendix D) agree well with the measured results. Only in some areas, e.g. above the Mt Isa Block and in the western part of the array, variations in the transfer functions are too incoherent to be modeled by large scale structures. Accordingly these areas are insufficiently sampled to model upper crustal structures.

For long periods (256 and 1024s) the modeled MT strike angles and phase differences (right column in Figure 6.2) agree well with the measured strike directions. Both are primarily related to the anisotropic structure in the lower crust and upper mantle. For short periods only small phase differences are observed in the modeled and measured results, indicating only minor $2 \mathrm{D}$ structures at shallow depth. This is related to conductive cover sequences in most parts of the study area that attenuate 
the effect of possible multidimensional structures below. Only in areas like the Mt Isa Block, where the basement reaches the surface larger phase differences at short periods can be observed.

\subsection{D inversion}

In contrast to forward modeling where the user modifies a model to fit the measured data, inversion schemes use computer algorithms for this alteration. For a limited number of parameters (e.g. in 1D inversions) the complete physical significant model space can be discretized and scanned to find the best fitting model (e.g. program 3layer1d in Appendix B). For more complex problems (2D or 3D structures) however, complete scans are impractical and other approaches have to be used. These include Monte Carlo methods as well as algorithms that systematically improve a given starting model in several subsequent steps. All approaches require solutions of the forward problem to estimate the model misfit.

Best fitting models of overparameterized problems like that of electromagnetic induction studies are usually rough and contain many features that are unresolved by the input data. Additional parameters like the model roughness or static shift parameters (Ogawa \& Uchida, 1996) can be used to reduce small scale heterogeneity. In addition regularizations that e.g. use the model roughness as additional modeling criteria can be introduced. While these regularizations are successful for smooth conductivity variations they lead to underestimated contrasts in case of strong conductivity gradients associated with tectonic boundaries. This can partly be overcome with additional modeling parameters (deGroot Hedlin \& Constable, 2004) and has to be considered in the interpretations of smooth inversion results. One particularly successful set of smoothing regularizations is used in the OCCAM approach (Constable et al., 1987; deGroot Hedlin \& Constable, 1990). Here the influence of the regularization on the final model can be controlled by the so called Lagrange multiplier.

The first 2D inversions for magnetotelluric data were published in the 1990s (deGroot Hedlin \& Constable, 1990; Ogawa \& Uchida, 1996; Siripunvaraporn \& Egbert, 2000; Rodi \& Mackie, 2001). Despite the long established theory for 3D electromagnetic inversion schemes the high computational requirements (in computational power and memory) made 3D inversion codes impractical in the past. Advances in computational power and new inversion approaches now allow the application of 3D inversion techniques on real datasets. For this study the OCCAM based 3D inversion code of Siripunvaraporn et al. (2005a,b) has been used. The application of the data-space approach allows this inversion to run on a normal PC. This implies the solution of the inverse problem (search for the best fitting smooth model) in the data-space of the input data values $(\mathrm{N})$ rather than the usually overparameterized model-space that contains all parameters $(\mathrm{M})$ of the resistivity model. This reduces the inverse problem to the solution of a $\mathrm{N} \times \mathrm{N}$ instead of a usually much larger $\mathrm{M} \times \mathrm{M}$ problem, saving computational time and memory in the process. Next to the in- 
version scheme itself the computational efficiency depends on the numerical forward problem solver. Siripunvaraporn et al. (2005a) use a fast finite difference method (including quasi-minimum residuals) that has been shown to be efficient even for large and complicated models (Siripunvaraporn et al., 2002).

Next to the input data several parameters control the inversion process. These include (i) the typical OCCAM Lagrange multiplier $\lambda$ that controls the smoothness of the inversion (ii) a model length scale that controls the decorrelation scale of the diffusion equation and (iii) the target RMS fit. The model length scale controls length scales of model structures in the three model directions (north, east, vertical) where large length scales allow variations on larger scales. The inversion runs in two phases. In phase 1 the misfit is reduced in subsequent steps and for different $\lambda$ values. When the target misfit is reached the program enters phase 2 where $\lambda$ is slightly perturbed to higher values to remove unnecessary structures from the model (Siripunvaraporn et al., 2005a).

So far the code allows inversion of the four complex impedance tensor elements. As real and imaginary parts are used for the inversion, static shift effects strongly influence the inversion. Therefore these effects have to be removed from the data. For the Australian dataset this was done with the help of the forward modeling results of the previous section and visual inspections of the apparent resistivities. While this handling is already questionable for the off-diagonal of the impedance tensor it is even more difficult for the poorer defined main diagonal elements. To work around this problem the error level of the main diagonal elements was increased by a factor of 5 to reduce their influence on the inversion. This is particularly important for the Australian dataset where the main-diagonal tensor elements of the overnight sites are not well resolved (compare Chapter 4). In principal the converted MT impedances obtained from the magnetic transfer functions (Chapter 5) provide another possible mechanism to remove static shift effects. For the Australian dataset however that approach has two limitations: First the TE-mode nature of the converted results in 3D environments (Chapter 2.5) and second a possible conductivity difference between the western and eastern part of the array. While the TE-mode nature could be dealt with by quasi-2D assumptions for the dataset (Appendix $\mathrm{C}$ ) a conductivity difference cannot be ruled out without additional measuring sites in the east.

The grid cell dimensions used for the inversion of the Australian dataset were $40 \times 40 \mathrm{~km}$ in lateral extent with thicknesses increasing from 0.8 to $50 \mathrm{~km}$ (18 layers) leading to a total model space of $24 \times 34 \times 18$ cells. These model dimensions were primarily limited by a memory allocation problem for large models. The large lateral block sizes hinder the inversion of short period data as structural differences between neighboring sites cannot be sufficiently accounted for. Therefore the inversion was confined to a period range of 128 to 2048 seconds. This is no limitation in the present case as the resolution of small scale local structures is already restricted by the large site spacings.

The final inversion results depend on both, the starting model and the chosen length scale. A good starting model should represent the major resistivities of the 
data without predetermination of unknown structures. Known structures like oceans however can be fixed in the model. For the Australian dataset where strong lateral resistivity contrasts are present, the best results were obtained with simple layered starting models and large model length scales (0.3-0.5 in $\mathrm{x}, \mathrm{y}$, and $\mathrm{z}$ ). In subsequent steps the model length scale is reduced to improve the model fit by allowing smaller scale structures, using the best fitting previous result as a starting model. For a starting model with $100 \Omega m$ in the top $4 \mathrm{~km}, 400 \Omega m$ from $4-48 \mathrm{~km}$ and $80 \Omega m$ for greater depth the effect of this procedure on the final model is illustrated in Figure 6.4. The first row shows the final model (7 inversion iterations, RMS 16.41) using a model length scale of 0.3 for all three dimensions. This model was used as input model for an inversion with model length scale of 0.1 (RMS $=11.86,3$ iterations, second column in Figure 6.4). This process was repeated with a model length scale of 0.03 (RMS $=5.81,6$ iterations, right column in Figure 6.4). From these models it is obvious that with decreasing model length scale smaller scale structures are included in the final model. At the same time stronger conductivity gradients are allowed. On the other hand the model roughness increases with decreasing model scale length. While the misfit could be reduced by even smaller length scales, these are not physically sensible in terms of data resolution and model roughness.

As a compromise between RMS and model roughness the model with a length scale of 0.03 was chosen as final 3D inversion model (right column in Figure 6.4). Using the original errors for the off-diagonal tensor elements and five times the measured errors for the main-diagonal elements the model fits the data with an RMS of 5.81. The model MT response and the corresponding measured results for two periods (128 and 1024s) are displayed in Figure 6.5. While the general patterns like the low phases in the south-east and high phases at 1024s above the Mt Isa Block are well resolved some differences, e.g. at site KAJ (measured phase above $90^{\circ}$ ) are obvious. For short periods these can be attributed to the coarse model parameterization while for longer periods they might be related to insufficient static shift removal in the input data. The imperfect fit at site KAJ shows that strong 3D effects in the data obviously cause problems for the inversion approach. As these are often related to small scale structures this is a general problem in MT modeling rather than a peculiarity of inversion schemes.

The final inversion model (right column in Figure 6.4) clearly show high conductivity areas in the south and east (top $3-4 \mathrm{~km}$ ) that are associated with the sediments of the Eromanga Basin. Following the model investigations of Siripunvaraporn et al. (2005a) the depth extent of conductive structures in a resistive host is often overestimated. Therefore these results do not contradict with sedimentary thicknesses of $1-3 \mathrm{~km}$. Furthermore a conductive bandlike structure following the Carpentaria anomaly is visible down to $30-40 \mathrm{~km}$ depth. The modeled resistivities of 1-50 $\Omega \mathrm{m}$ match those found by Lilley et al. (2003) for the northern part of the array. The Mt Isa Block is represented as resistive crustal block with significantly larger lateral extent (towards the south and west) than inferred from surface geology. At upper mantle depth (from $68 \mathrm{~km}$ downwards) an elongated east-west striking conductive structure becomes evident. This defines a similar structure to the anisotropic upper 

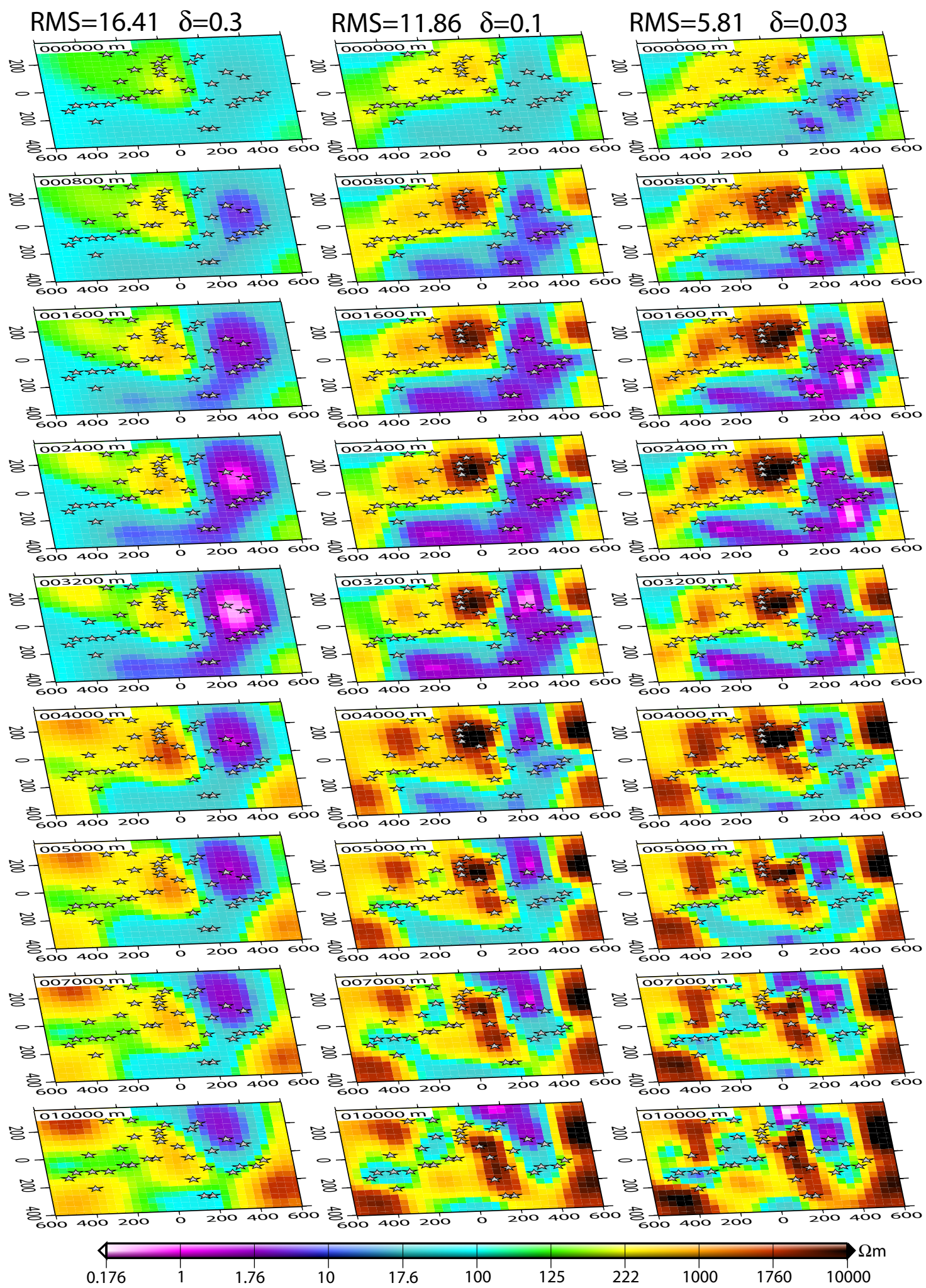

Figure 6.4: Upper 9 layers of the best fitting inversion models for 3 different model length scales $(\delta=0.3,0.1$ and 0.03 ). The best model of the longer length scale was used as input model for the next smaller scale length. Stars indicate the model site positions. (Figure continues on the next page) 

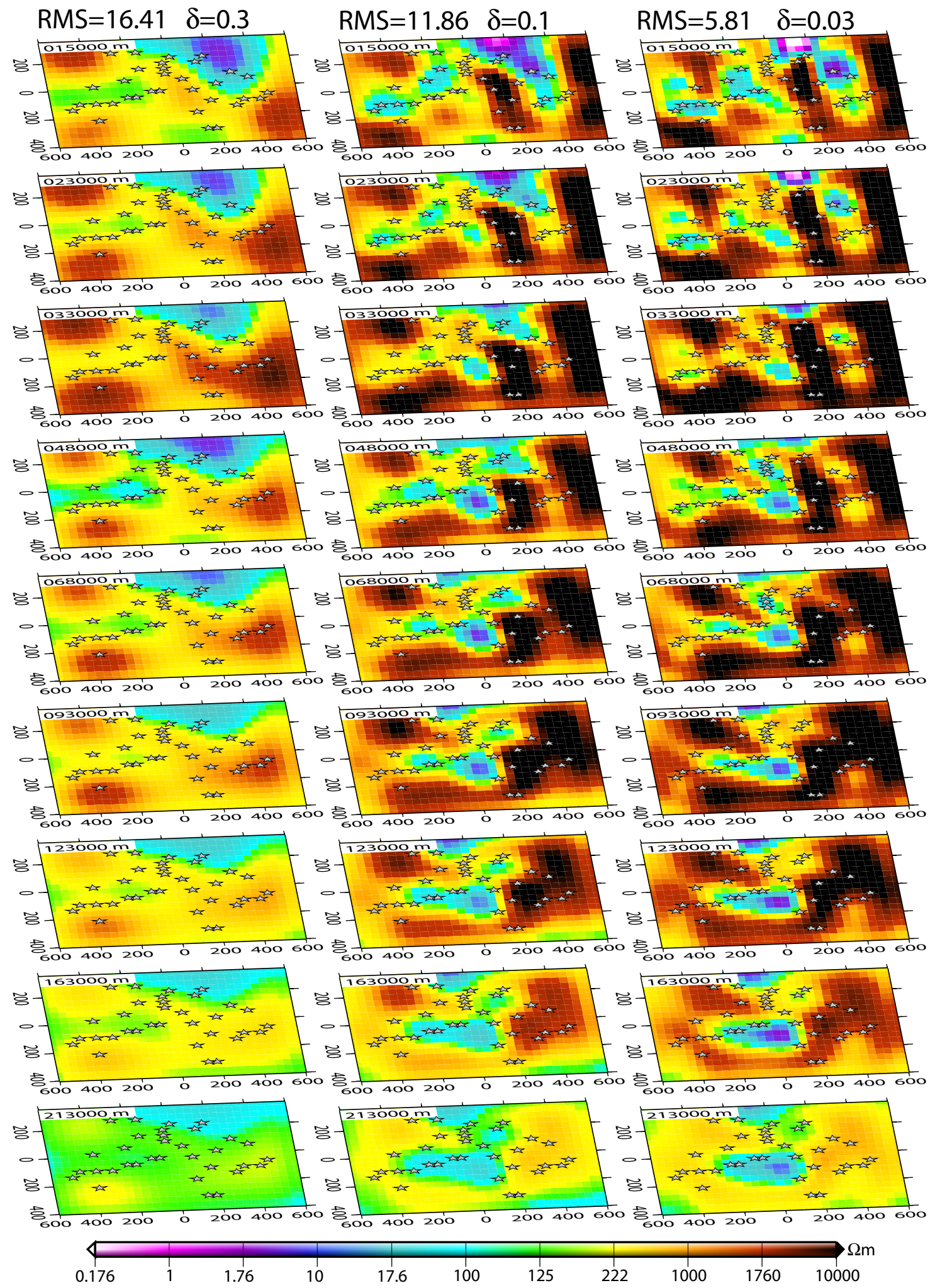

Figure 6.4: (cont.) Lower 9 layers of the models. The column to the right shows the final 3D inversion model with model length scale of $\delta=0.03$ and an RMS of 5.81 . 


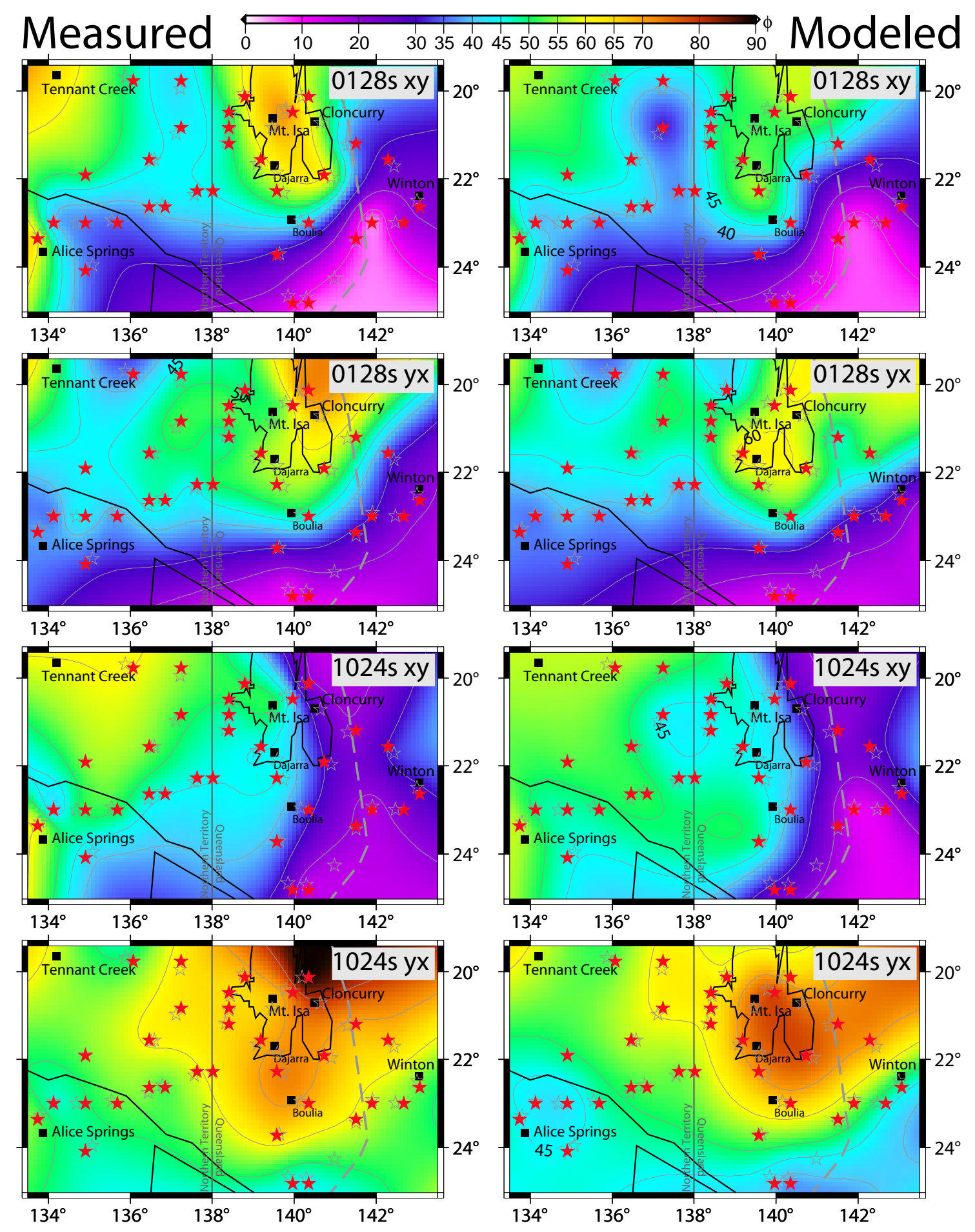

Figure 6.5: Measured (left) and modeled (right) phases of the off-diagonal tensor elements for periods of 128 and 1024 seconds. The red stars indicate model sites while the grey stars show field site positions. The location inconsistencies arise from the coarse model parameterization. 
mantle included in the forward model of the previous Section. The limitation of the period range to 2048 seconds, however, does not lead to well resolved mantle structures particularly below the sediments of the Eromanga basin. More detailed investigations of mantle structures using the full period range will be carried out in a separate study.

Other striking features are the high resistivities $(>1000 \Omega m)$ found in the lower crust and upper mantle. These are higher than expected from other investigations in Australia (Campbell et al., 1998) and might be artefacts related to the low structural resolution below the conductive sediments.

Despite influences of static shift effects in the input data and the limitation to MT data only, the inversion results are consistent with the forward modeling results and expected geological structures. The fact that these structures were not included in the starting model strengthens the confidence in the final model. Only strong 3D effects in the input data are not convincingly represented by the inversion results.

\subsection{Structural and geological implications of the models}

The differences between the 3D forward model and the 3D inversion results are primarily based on the different modeling philosophies. Least structure models are designed to have strong resistivity contrasts to minimize structural diversity while the applied inversion algorithm searches for smooth models. Nevertheless several common features can be found. These include (i) conductive sediments, (ii) resistive structures in the area of the Mt Isa Block that are underlain by anisotropic or large scale two-dimensional structures in the lower crust or upper mantle and (iii) a conductive band representing the Carpentaria anomaly that extends to $30-50 \mathrm{~km}$ depth.

\subsubsection{Sediments of the Eromanga Basin}

Both, the forward model and the inversion results clearly indicate highly conductive areas in the southern and eastern parts of the final model. These are related to the sediments of the Eromanga Basin that cover large parts of central Australia. The conductivity of young sediments is dominated by the conductivity of pore fluids and the porosity of the sediment. As the Eromanga Basin hosts part of the Great Artesian Basin, central Australia's largest water reservoir (GABCC, 2000) a high fluid content of the sediments can be implied. Archie's Law (Reynolds, 1997)

$$
\rho=a \phi^{-m} s^{-n} \rho_{w}
$$

gives an empirical formula to calculate the effective resistivity of the sediments $\rho$ from the porosity $\phi$, the volume fraction of the pores with water $s$ and the pore fluid conductivity $\rho_{w}$. $a, m$ and $n$ are parameters that vary $0.5 \leq a \leq 2.5,1.3 \leq$ $m \leq 2.5$ and $n \approx 2$. Therefore measured artesian bore water conductivities of 
up to $10 \mathrm{~S} / \mathrm{m}$ can easily explain the modeled resistivities between 1-10 $\Omega \mathrm{m}$. The final models indicate sedimentary thicknesses of more than $2500 \mathrm{~m}$ in the southeastern part of the array with reduced thicknesses towards the north. Despite the limited spatial resolution of this study these results are in agreement with other investigations (GABCC, 1998; Clitheroe et al., 2000a; Lilley et al., 2003). For more detailed structural analysis of the basin, densely spaced, high frequency (shallower penetration depth) electromagnetic data are required.

\subsubsection{Mt Isa Block}

According to geological investigations the Mt Isa Block has a diverse and manifold tectonic history including several compressional, extensional and intrusive events. The structural grain of the block was formed during the Barramundi Orogeny in a back-arc rift environment (1.9-1.87 Ga, Betts et al. (2006)). During the Isan Orogen (1.6-1.55 Ga, Giles et al. (2006)) major parts of the Mt Isa Block were formed in a compressional regime that was proposed to include subduction of oceanic crust between colliding continental blocks (Betts et al., 2006). This is supported by seismic refraction data indicating a westward dipping high velocity layer throughout the Mt Isa Block (MacCready et al., 1998; MacCready, 2006). These multiple tectonic events lead to a diverse geological structure with various fault systems and intrusions that should be represented in the geoelelectric structure of the inlier. While short period geoelectric strike directions (Section 4.1.1) agree with the predominant northerly strike of the Isan orogeny (Betts et al., 2006) the sparse data coverage does not allow upper crustal structural investigations of the Mt Isa Block. However the modeling results of this study allow regional scale investigations.

Both, forward and inversion model resolve the Mt Isa Block as a highly resistive crustal unit. These high resistivities (1000-10000 $\Omega \mathrm{m}$ ) are related to the crystalline basement and are common for Proterozoic crust (Boerner et al., 1999). Below shallow covering sediments the Mt Isa Block has a significantly larger extend (towards the south and west) than inferred from surface geology. This is in agreement with previous gravity and magnetic anomaly investigations (Chapter 3). The resistivity of the block decreases with depth. In the 3D forward model an anisotropic layer was modeled from $75 \mathrm{~km}$ downwards to fit the strong MT phase split at longer periods. The inversion fits this phase split with east-west striking macroscopic structures at the northern border and in the center of the model (from $50 \mathrm{~km}$ downwards). Both models equally fit the measured data as the MT method is not generally able to distinguish between microscopic anisotropy on crystal scale and large scale heterogeneous structures (termed anisotropic in the following) (Eisel \& Haak, 1999). The confinement of the structure below the MT Isa Block suggests a causative relation to the creation or alteration of the MT Isa Block. The east-west focus of the higher conductive direction might be related to compressional or extensional events during the evolution of the Mt Isa Block (Griffin et al., 2006; Giles et al., 2006; Bierlein \& Betts, 2004; Betts, 1999). Opposing geological interpretations and the multitude of structure forming events however do not allow an attribution to a certain tectonic 
event. Due to the focus on crustal structures the present model study was confined to periods up to 1024s (forward modeling) respectively 2048s (inversion). As the modeling indicates upper mantle depth for the anisotropic structure further insight into the origin and depth extend of the structure will be obtained from long period investigations of the present dataset. These will be performed in a different study.

\subsubsection{Carpentaria conductivity anomaly}

The discovery and location of the Carpentaria conductivity anomaly was described in some detail in Chapter 3.6. These results influenced to a certain extend the initial forward model. GDS and local induction arrow results however require the inclusion of a conductive band at the eastern boundary of the Mt Isa Block. This is supported by the GDS to MT conversion results of Chapter 5 that reveal the similarity between these electromagnetic transfer functions. While models with continuously decreasing resistivities towards the west are able to explain the local induction arrows in the western part, these models provide poor fits for the reversal of the arrows above the anomaly and for the other measures. This illustrates the advantage of joint interpretations of all available quantities, in particular when the consistency between the datasets was verified beforehand by bidirectional data conversions as in Chapter 5 .

While the lateral location of the Carpentaria anomaly was previously known from electromagnetic (Chamalaun \& Barton, 1993; Chamalaun et al., 1999), gravity and aeromagnetic investigations (Chapter 3) only limited knowledge exists about the lateral variations of the depth extent and origin of the anomaly (Wang \& Lilley, 1999; Lilley et al., 2003). As a major continent scale electromagnetic structure, the Carpentaria anomaly was most likely formed by tectonic processes, possibly during the construction (1.3-1 Ga) or breakup (0.8-0.55 Ga, Wingate et al. (2002)) of the early supercontinent Rodinia (Myers et al., 1996; Giles et al., 2004). Such interpretations are hampered by the long and diverse tectonic history of the Mt Isa Block discussed in the previous section. However, subduction zones and continentcontinent collisions have been shown to produce conductivity anomalies (e.g. Soyer \& Brasse (2001); Li et al. (2003)) and are therefore a promising candidate for the origin of the Carpentaria anomaly.

Based on geochronological and paleomagnetic measurements several reconstructions of the Proterozoic Australian continent have been published (Karlstrom et al., 2001; Wade et al., 2006; Betts et al., 2002; Giles et al., 2004). Most of theses date the amalgamation of the north, west and south Australian cratons before 1.5Ga. Giles et al. (2004) and Spence \& Finlayson (1983) however propose a reconstruction where the south Australian craton (compare Figure 3.1a) broke away from proto-Australia after $1.5 \mathrm{Ga}$ only to be reattached in the present position during the 1.33-1.1 Ga orogeny of south-eastern Australia. This model brings the Curnamona province (Broken Hill) in connection to the geologically similar eastern Mt Isa Block, combining both to a north-south trending orogenic belt (Figure 6.6b). Assuming that the Carpentaria conductivity anomaly was created by this event the 

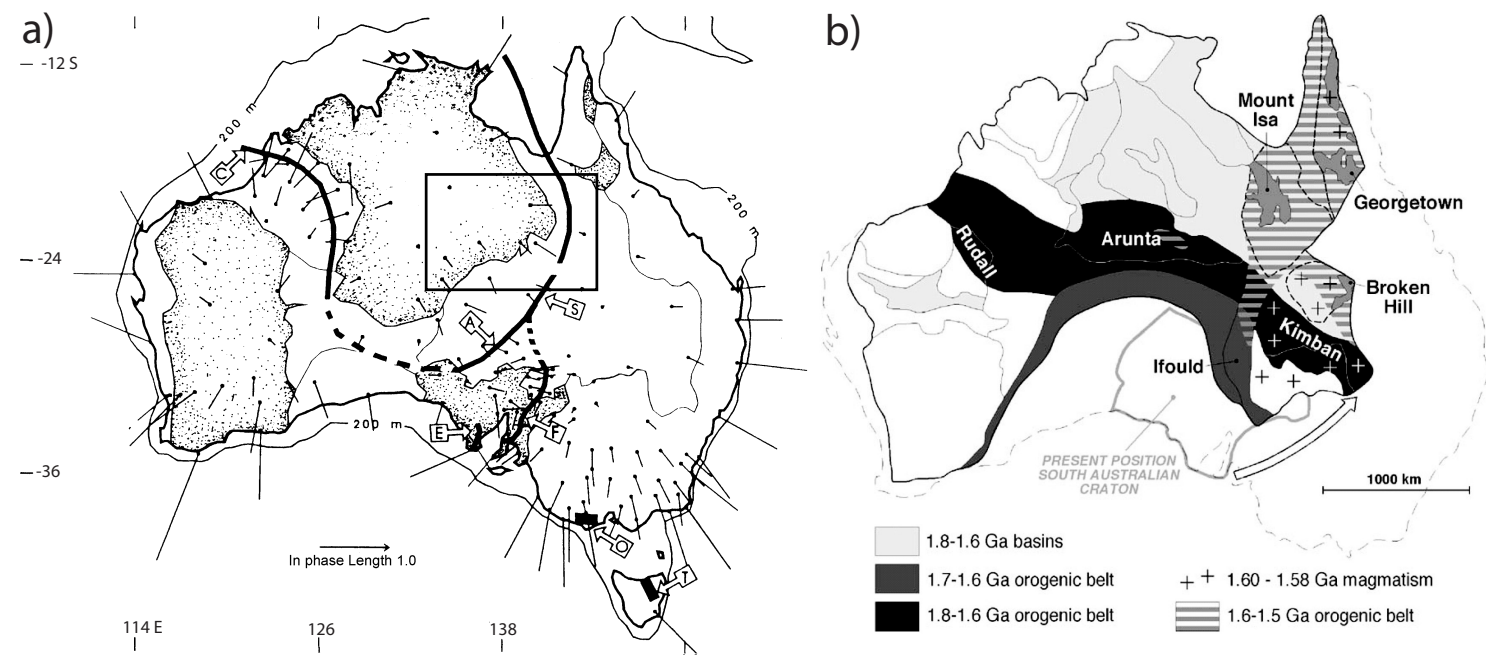

Figure 6.6: (a) Real induction arrows (Parkinson convention!) of the AWAGS experiment. The solid lines mark major crustal conductors defined from the magnetic measurements. The box marks the survey area of this study (modified from Chamalaun \& Barton (1993)). (b) Reconstruction of Palaeozoic Australia by Giles et al. (2004). In this reconstruction the south Australian craton is rotated by $52^{\circ}$ with respect to its present location.

rearrangement connects it to the Flinders Range conductivity anomaly found by Chamalaun \& Barton (1993) (Line F in Figure 6.6a), supporting their hypothesis of continent scale conductivity anomalies along major structural boundaries.

The repeated tectonic reactivation of various Proterozoic terranes is one of the main features of the Proterozoic evolution of central Australia. Following McLaren et al. (2005) this is a consequence of elevated heat flow that leads to long-term lithospheric weakening in central Australia. Following seismic constraints on mantle temperatures the heat flow anomalies in central Australia are not produced by increased mantle temperatures (Goes et al., 2005) but a higher degree of upper crustal Proterozoic lithosphere with higher than the worldwide average radiogenic element contents (McLaren et al., 2005). The elevated heat production is attributed to high heat-producing element concentrations in Proterozoic crust that is preserved in Australia as a result of low denudation rates in central Australia (McLaren et al., 2003).

These models explain the creation of regional scale structural boundaries in the area of the Carpentaria anomaly but do not define the mechanism for its observed elevated conductivities. Primarily two conduction mechanism leading to crustal conductivity anomalies are discussed in the literature: (i) conductive fluids (including partial melt) or (ii) interconnected graphite layers. In many tectonically active regions partial melts and fluids provide the most important conduction mechanism (Li et al., 2003; Unsworth \& Bedrosian, 2004). In very old crustal units however fluids 
are too unstable to sustain time spans of billions of years. Therefore interconnected graphite layers originating from tectonic stresses (Nover et al., 2005; Roberts et al., 1999) or precipitated from fluids during tectonically active times (Kellet et al., 1992) are the most promising candidates for a high conductivity phase in the Carpentaria anomaly. This is in agreement with recent MT results from the Central Australian Suture, another major crustal boundary in several geophysical parameters (Selway et al., 2006).

The fact that the Carpentaria anomaly divides the Proterozoic Mt Isa and Georgetown inliers (Figure 6.6b) might indicate that the Georgetown Inlier was not part of the early-Proterozoic North Australian Craton but accreted as an independent crustal fragment, possibly during the Isan Orogeny (1.6-1.55 Ga). This is in contradiction to basically all reconstructions of Proterozoic Australia where both inliers were connected well before $1.5 \mathrm{Ga}$ (e.g. Karlstrom et al. (2001); Wingate et al. (2002)). Recent morphological investigations however exhibit significantly differing metamorphic evolutions of the Georgetown and Mt Isa Inlier (Boger \& Hansen, 2004). In combination with the electromagnetic models of this study this implies an independent development of the two inliers that were amalgamated at later times possibly during the Isan orogeny around $1.5 \mathrm{Ga}$ or during the formation of Rodinia some $100 \mathrm{Ma}$ later. This interpretation is supported by the seismological investigations of Clitheroe et al. (2000b). They found a distinct transition between thick crust beneath central Australia to thinner crust below eastern Australia that separates the Georgetown and Mt Isa inliers (gray line in Figure 3.3).

\subsubsection{Tasman Line}

The Carpentaria anomaly coincides with the boundaries of prominent gravity and magnetic anomalies that have been used to define the location of the Tasman Line at the southern boundary of the Mt Isa Block (Veevers, 1984; Gunn et al., 1997). At the eastern boundary of the MT Isa Block however the Carpentaria anomaly follows the major gravity and magnetic gradients towards the north (Figures 3.5+3.6) while the Tasman Line of Gunn (1997) continues in north-easterly direction to include the Proterozoic Georgetown Inlier into the North Australian Craton. Due to the different ages of the Carpentaria anomaly, that was created prior or during the formation of the supercontinent Rodinia (before $1.1 \mathrm{Ga}$ ) and the Tasman Line that is referred to as the Rodinian breakup boundary (0.8-0.55Ga) no causative connection between their formations should exist. This implies that if the boarders of the magnetic and gravity anomalies at the Mt Isa Block are related to the creation of the Carpentaria anomaly they are significantly older than the Rodinian breakup. While the repeated reactivation of tectonic structures in central Australia might have been a common feature in the Proterozoic this weakens possible correlations of magnetic and gravity anomalies with the Tasman Line.

As the boundary between the young crust of eastern Australia and Proterozoic central Australia, the Tasman Line could potentially by resolved by electromagnetic methods as (i) a conductivity anomaly along the boundary between two crustal 
fragments or (ii) by a conductivity contrast between the old (resistive) Proterozoic parts and younger (more conductive) eastern Australia.

A clear statement about the Tasman Line from the dataset of this study alone is difficult as virtually no sites were measured east of the Carpentaria anomaly. However, the forward model contains higher conductivities to the east of the Carpentaria anomaly. This allows a better fit for the induction arrows that are slightly smaller in the east than in the west of the anomaly. For a conductive bandlike structure in a homogenous surrounding the induction arrow pattern is symmetric while different resistivities to the sides lead to smaller arrows on the conductive side. The sparse site sampling and the strong lateral induction arrow variations close to the anomaly hinder the interpretation. The inclusion of the induction arrows of Chamalaun et al. (1999) (Figure 3.7) however support higher conductivities in the east. Similar effects could be produced by the resistive Mt Isa Block and the coast effect of the coral sea in the east. Therefore more detailed investigations that extend the existing array to the east are required to get further insight into possible Tasman Line related conductivity structures and the location of the Rodinian breakup boundary.

An independent formation of the Georgetown and Mt Isa inliers as discussed in the previous section implies more structural diversity in north-eastern Queensland than previously assumed. The possibility of accretion of older crustal fragments to the North Australian Craton in the late Proterozoic not only complicates the reconstruction of the early supercontinent Rodinia but as well the definition of the Tasman Line as Rodinian breakup boundary. In that case the concept of a simple Tasman Line may not even be applicable to Queensland where it was originally defined. This supports the suggestion of Direen \& Crawford (2003) to abandon the concept of a simple Tasman Line. 


\section{Conclusions}

The dataset presented in this study provides the unique opportunity to image the large scale conductivity structure of wide parts of central Australia. The total extend of the area covered by the measurements is $600 \times 900$ kilometers. The typical site spacing is $50 \mathrm{~km}$ while the limited accessibility of some areas lead to variations in lateral coverage. Magnetic (GDS) and magnetotelluric (MT) transfer functions have been measured and were combined to a joint dataset utilizing the different natures of the transfer functions: Magnetic transfer functions are sensitive to currents flowing in the earth while the electric fields used in MT are controlled by current flow and quasi-static electric fields created by charge accumulation at conductivity contrasts. The conversion of magnetic to magnetotelluric transfer functions (Becken \& Pedersen, 2003) allows a separation of both effects and therefore simplifies the interpretation of electromagnetic data. While the original algorithm was developed for airborne VLF data this study applies the algorithms on long period MT and GDS data. In order to investigate specific features of the conversion it was extended to allow for the conversion of horizontal magnetic (rather than induction arrow only) to MT transfer functions. Inverse conversions can be used to test the algorithm. Despite the limitation on 1D background structures (no conductivity contrasts at the array boundaries) good conversion results were obtained for the Australian dataset and array data from Germany. In particular for multidimensional structures this conversion allows for the identification of electric distortion related TM-mode effects.

In combination with other geophysical (i.e. gravity, aeromagnetic, seismological) investigations electromagnetic data can be used to define the major structural features of the central Australian crust. This is done by 3D forward modeling of all transfer functions and 3D inversion of the MT results. The models clearly define the major crustal structures of the region: (i) the crystalline Mt Isa Block, (ii) the Eromanga Basin and (iii) the Carpentaria conductivity anomaly. In agreement with regional gravity and magnetic anomaly data the models reveal that the Mt Isa Block extends several $100 \mathrm{~km}$ further south than inferred from surface geology. At depth of more than $50 \mathrm{~km}$ below the Mt Isa Block the models show direction dependent conductivities, possibly remains from tectonically active times of the area. The Eromanga Basin is up to $3 \mathrm{~km}$ thick and consists of highly conductive sediments. The modeled resistivities of less then $1 \Omega m$ are related to the mineral rich, conductive fluids of the Great Artesian Basin. The western part of the covered area consists of a resistive Proterozoic basement that is covered by sediments of moderate resistivity. One of the most prominent features in the raw data and the final models is the Carpentaria conductivity anomaly. This high conductivity bandlike structure follows the eastern border of the Mt Isa Block and extends to depths of 40-50km. 
The location between the Proterozoic Mt Isa and Georgetown Inliers indicates independent formations of the inliers. Despite the contradiction to previous Proterozoic plate reconstructions this hypothesis is supported by geochemical evidence showing different metamorphic evolutions of the inliers (Boger \& Hansen, 2004). The collision of these crustal units during the Isan orogeny $(1.5 \mathrm{Ga})$ or the formation of the early supercontinent Rodinia $(\approx 1.3 \mathrm{Ga}$ ) provides a logical source mechanism for the creation of the conductive Carpentaria anomaly.

The Tasman Line as defined by geological, magnetic and gravity anomaly data follows the Carpentaria anomaly at the southern border of the Mt Isa Block. It is commonly brought in context with the breakup of the early supercontinent Rodinia. However, if the hypothesis of an independent evolution of the Mt Isa and Georgetown Inliers is correct and the Carpentaria anomaly was created during their collision several 100Ma of tectonic activity separates the creations of the Carpentaria anomaly and the Tasman Line. In addition the independent evolution of the inliers complicates the geological architecture of north-eastern Australia thereby covering up possible locations of the Tasman Line. Both arguments support the recently discussed questioning of the simple Tasman Line concept.

As a transition between younger crust in the east and the Proterozoic basement of central Australia electromagnetic techniques could resolve the Tasman Line as a large scale conductivity contrast. While the local induction arrow pattern of this project might indicate higher conductivities south-east of the Mt Isa Block additional measurements in eastern Australia are required to confirm this.

Major parts of central Australia's crust show moderate lateral resistivity variations and can therefore be resolved with a site spacing of $50 \mathrm{~km}$. Only heterogenous crustal structures like the Mt Isa Block with its exposed basement are to diverse to be investigated in detail by the present study. In those regions much smaller site spacings are required to fully resolve the local structures.

The results of this study illustrate the strength of 3D electromagnetic array studies. In contrary to profile measurements, 3D studies allow more accurate positioning of major boundaries and structures. Even with the sparse site spacing of this study the general structural features of central Australia could be resolved. Improved structural resolution can often be obtained by combination with other geophysical, geochemical and geological methods. While these measurements investigate independent physical properties of the Earth, major regional boundaries often show distinct signatures in several parameters.

Array data are particularly useful in combined MT and GDS surveys as they allow, within certain restrictions, conversions of one dataset into the other. This is partly due to redundant information in the datasets. Differences between converted and measured results however can be used to reveal the origin of anomalies as inductive features are common to both datasets while charge accumulations at conductivity contrasts effect the electric field only. In that context transformations of magnetic to magnetotelluric transfer functions can be used to visualize common and independent features in the datasets. 


\section{A Field sites and data}

\section{A.1 Locations and operating times of the MT sites}

\begin{tabular}{l|l|l||l|l|l} 
station name & longitude & latitude & station name & longitude & latitude \\
\hline ALD* & 139.736 & -22.327 & HAR & 134.690 & -23.005 \\
AMM & 134.911 & -21.896 & HEA* & 138.328 & -21.179 \\
ARD & 139.096 & -21.662 & HUC* & 135.551 & -22.937 \\
ARG* & 136.513 & -21.585 & ISA & 139.796 & -20.403 \\
ARGAMT & 136.585 & -21.566 & JER & 136.439 & -22.762 \\
ARL & 133.800 & -23.282 & KAJ* & 140.188 & -20.122 \\
BAR & 138.448 & -20.540 & KOO* & 139.853 & -24.645 \\
BHS & 135.884 & -19.668 & MON* & 139.586 & -21.853 \\
BRE & 139.640 & -23.703 & MTE & 142.431 & -21.720 \\
CAM & 138.762 & -20.051 & NAS & 137.110 & -20.979 \\
CLO & 140.635 & -20.719 & NUM* & 135.074 & -23.954 \\
CLU & 140.301 & -24.681 & RID* & 134.132 & -22.960 \\
COP* & 139.464 & -20.832 & SOU* & 137.223 & -19.946 \\
COR & 141.909 & -22.920 & TAR & 136.781 & -22.591 \\
DAV & 140.979 & -24.243 & TOB & 137.996 & -22.288 \\
DIA* & 141.513 & -23.437 & TOO & 140.900 & -21.975 \\
EUL* & 141.306 & -21.229 & URL & 137.560 & -22.365 \\
EXT & 138.412 & -20.875 & WES* & 140.162 & -22.964 \\
HAP & 142.475 & -22.997 & WIN* & 143.000 & -22.495
\end{tabular}

Table A.1: Station names and coordinates of the MT sites. Asterisks mark sites with more than 6 days of data.

Due to problems with the instrumentation, especially the incompatibility of the cables of the instruments with cow and camel dentition some or all field components of some sites were lost. Dried out electrodes were a problem at other sites. The most severe problems were:

- the loss of the electric fields due to drift in both components at site DAV

- loss of magnetic and electric components at site MON after $15 \mathrm{~h}$

- loss of the electric fields at site COP 


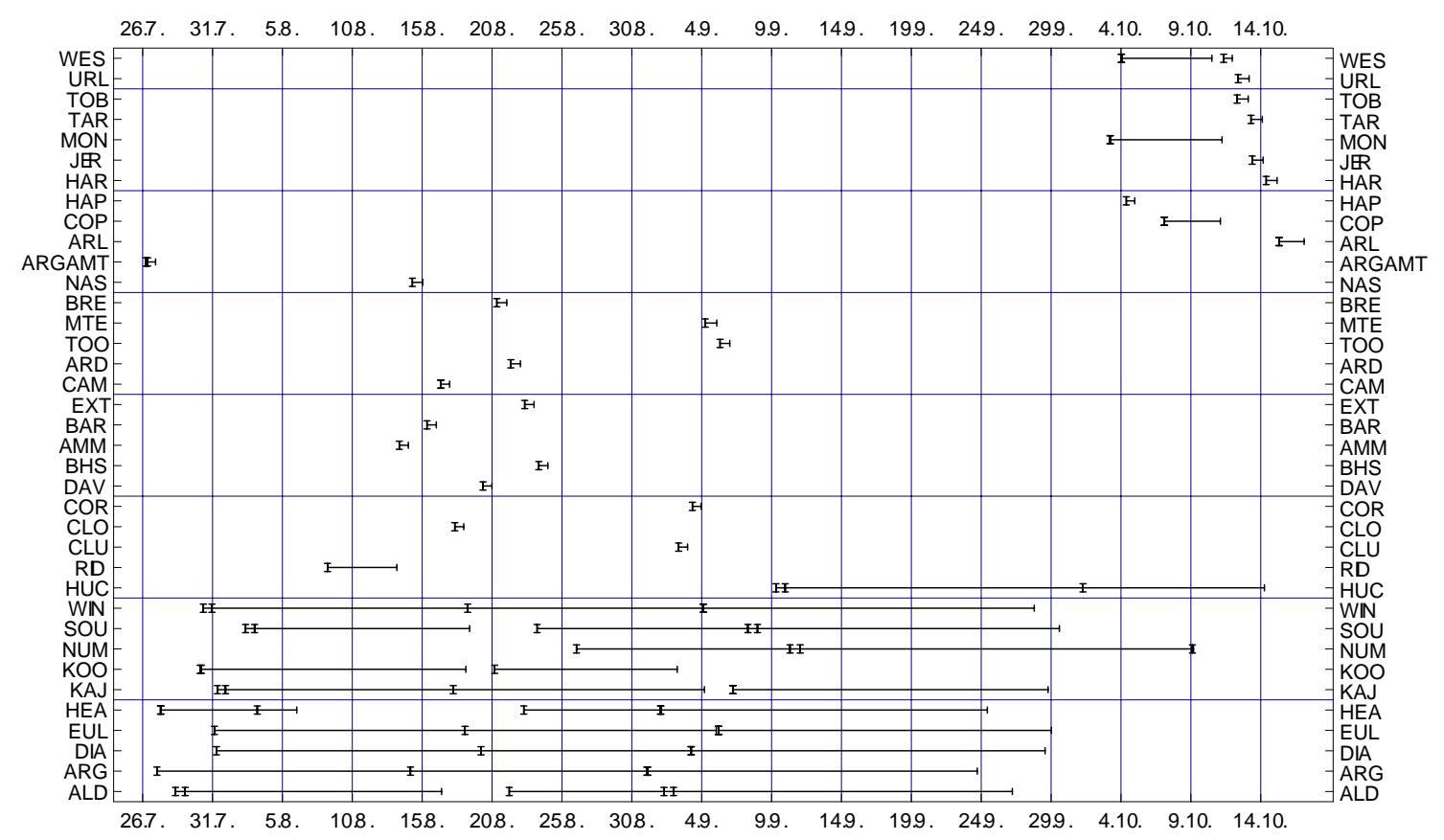

Figure A.1: Running times of the MT sites. Vertical bars mark the beginning and end of single recordings between site servicing.

\section{A.2 Processed MT data}

Figures A.2-A.4 plot apparent resistivities and phase values of the off-diagonal impedance tensor elements for all sites of the central Australian campaign 2003 in an unrotated (North-South/East-West) coordinate system. The different period range is related to the different operating times. For site NUM short period data obtained by a modified RAP data-logger (Jantos, 1999) with induction coils as magnetometers is plotted in addition to the long period results.

\section{A.3 GDS results}

Figures A.5 and A.6 plot all real parts of the GDS perturbation tensor as interpolated map plots for periods from 32-5464 seconds. For each graph all sites marked by red stars have been included in the interpolation procedure. Site ARG has been used as reference station. 


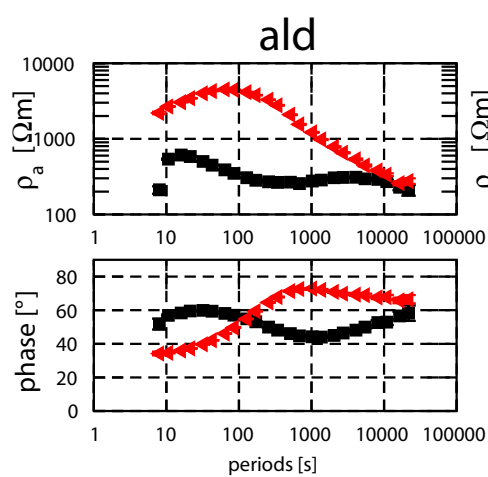

$\arg$
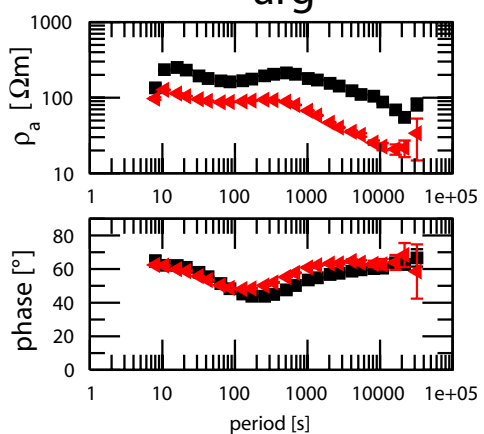

bhs
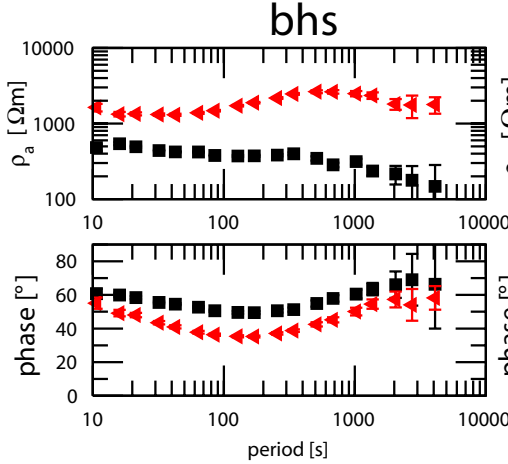

clo
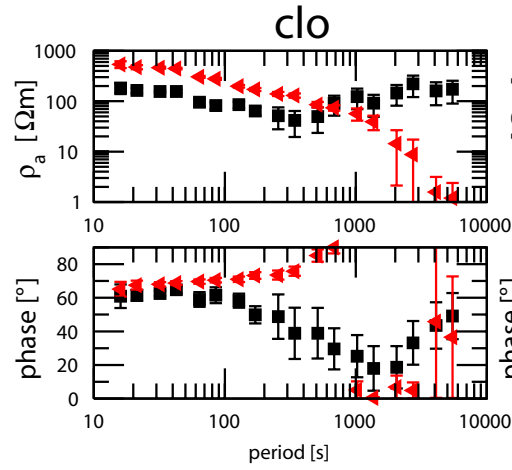
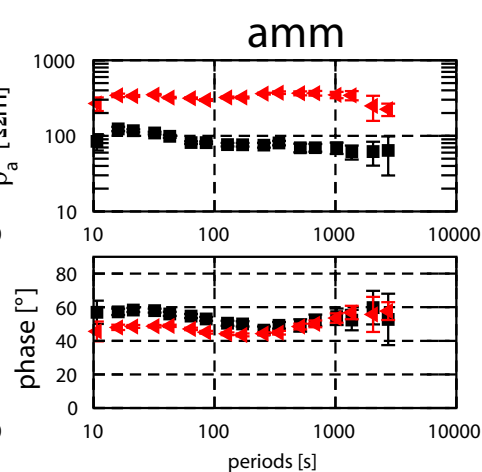

arl
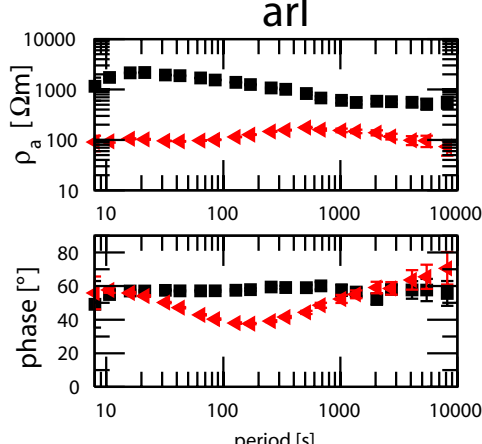

bre
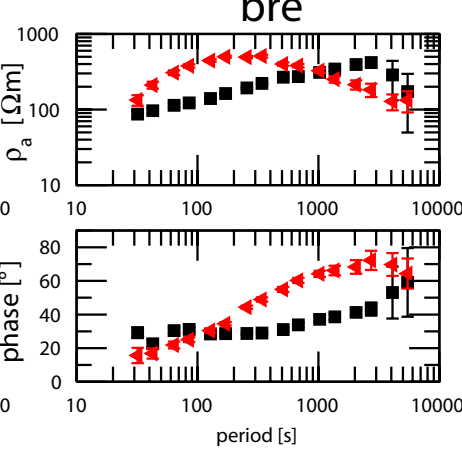

clu
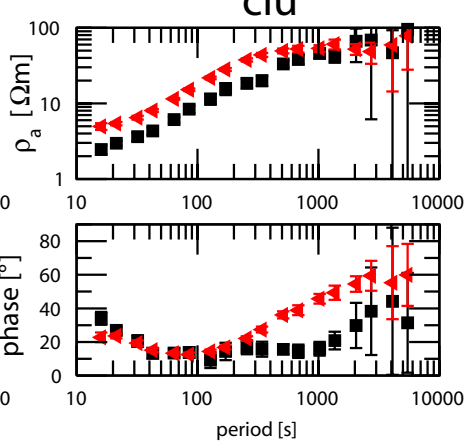
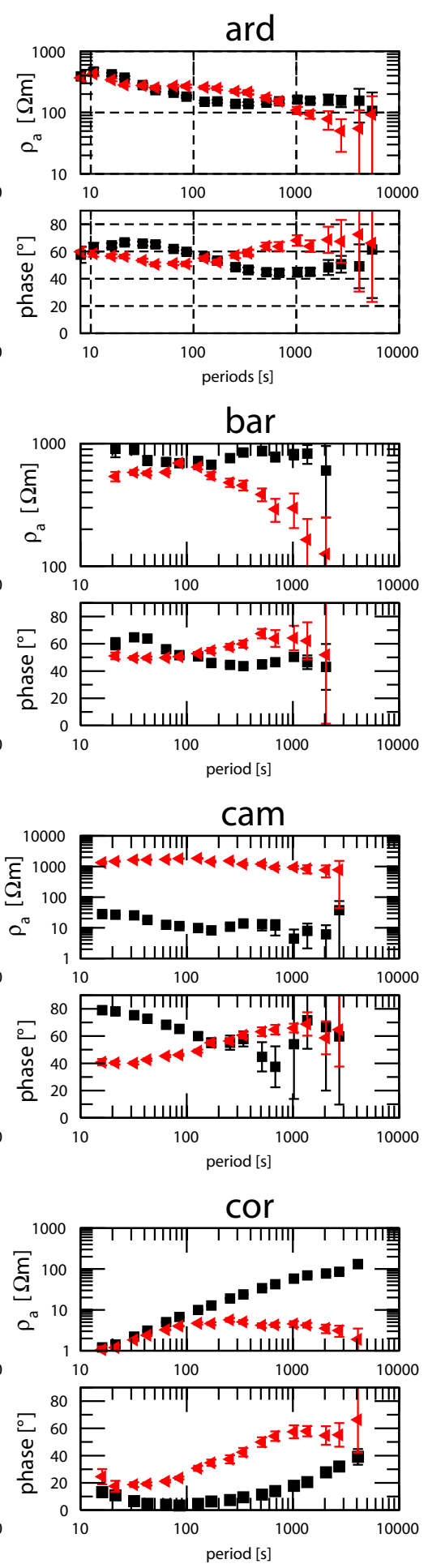

Figure A.2: Unrotated apparent resistivity and phase curves for the MT sites of this study. Black squares indicate xy and red triangles yx component. 

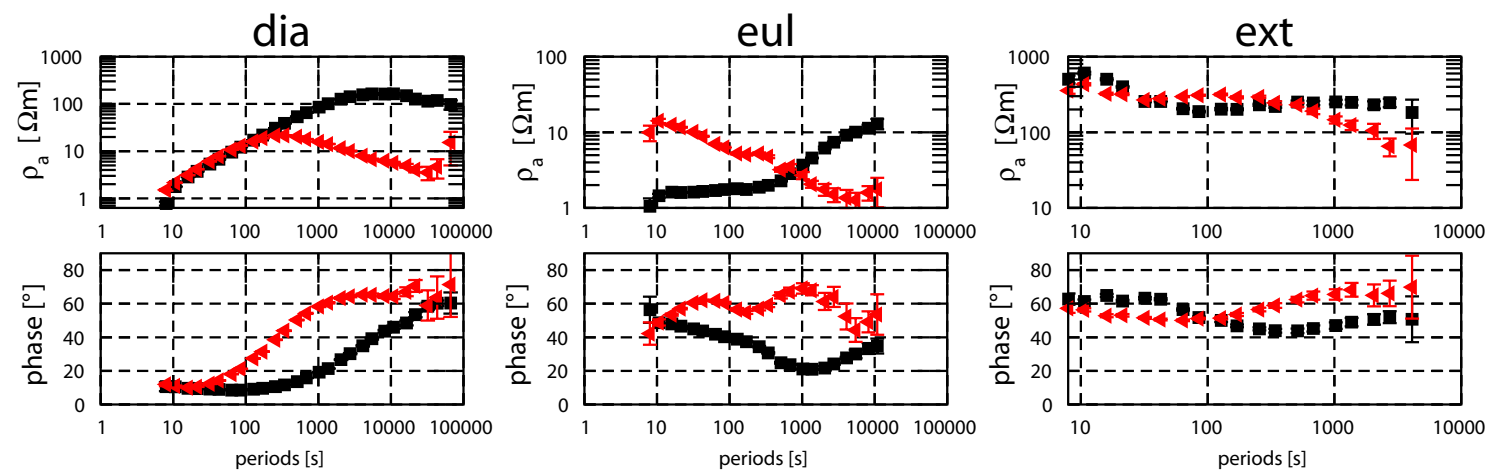

hap

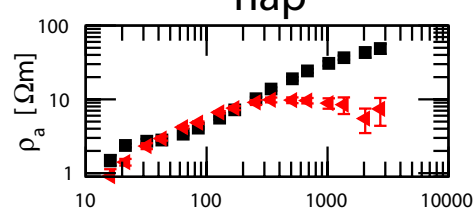

har

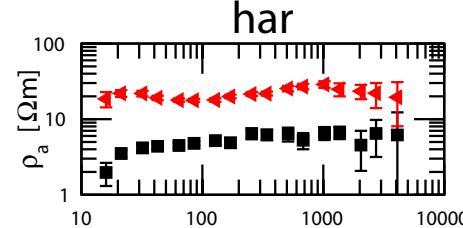

hea
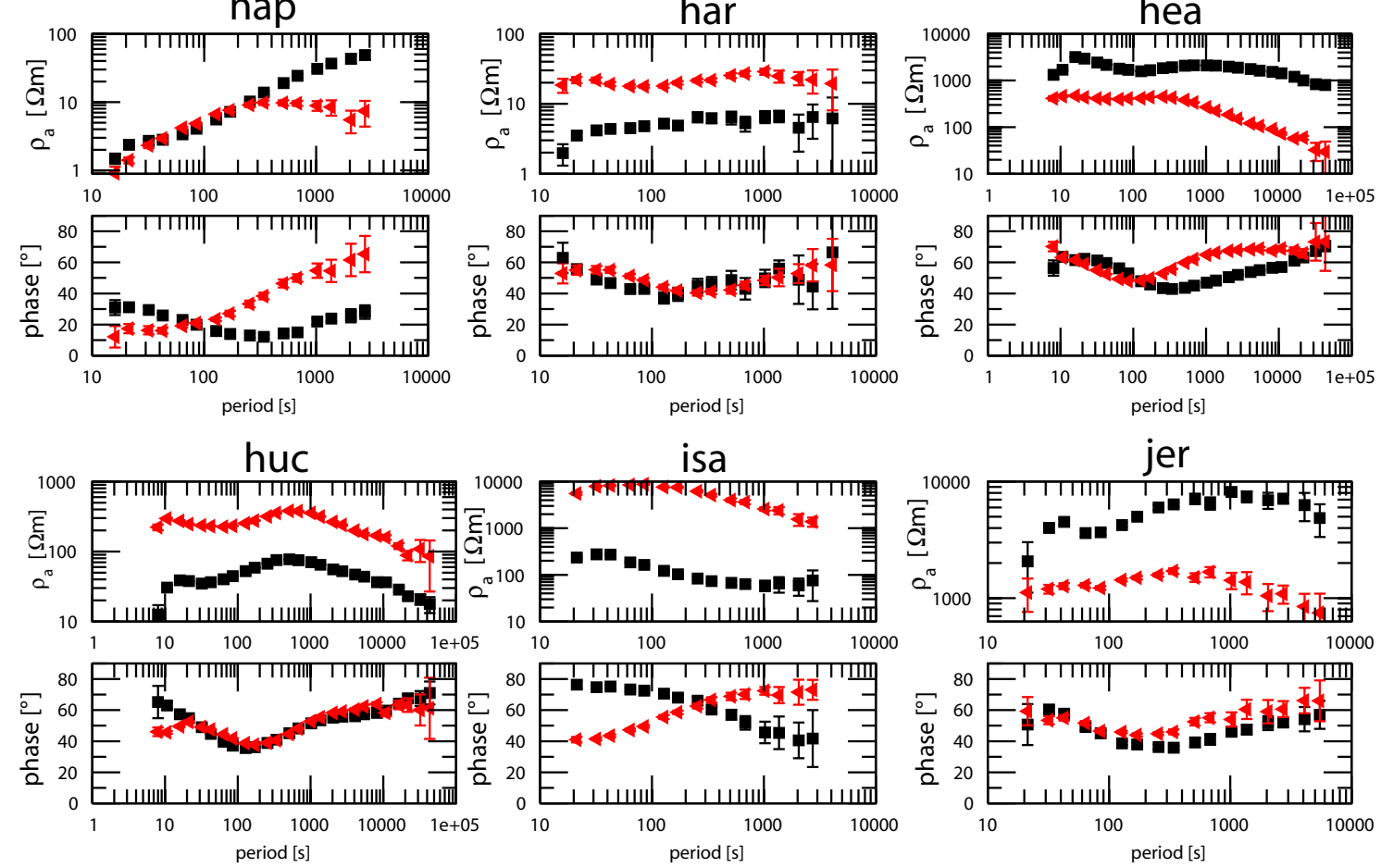

kaj

koo

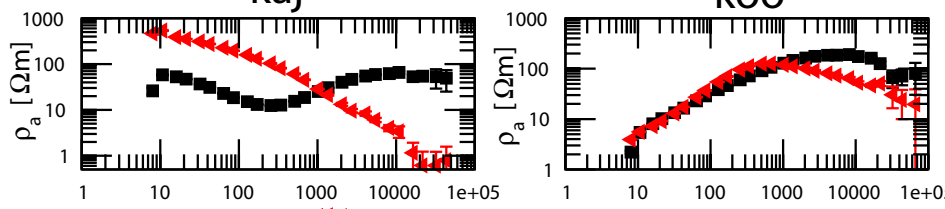

mon
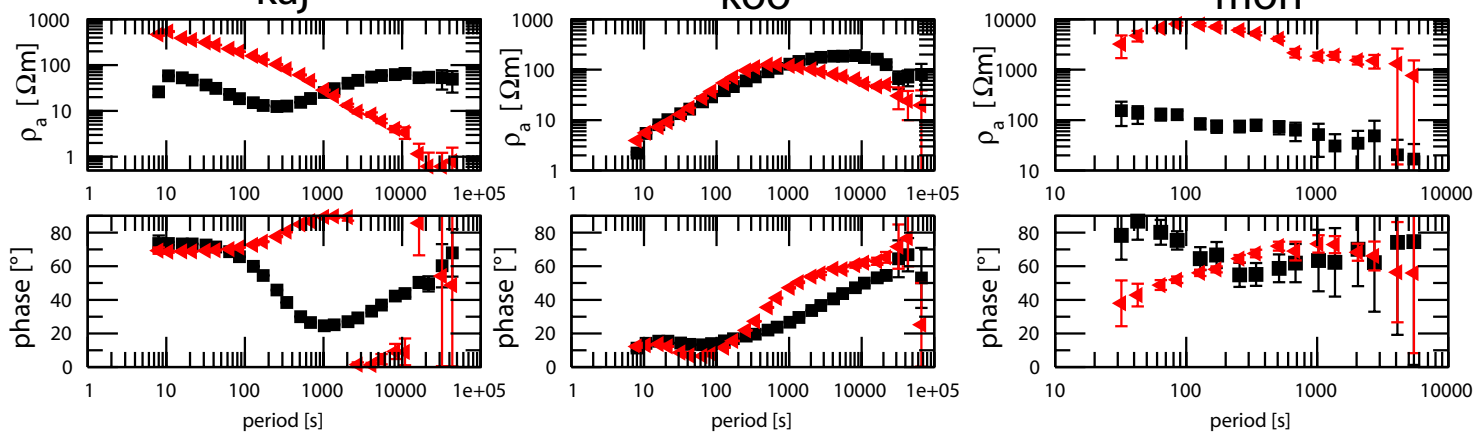

Figure A.3: Unrotated apparent resistivity and phase curves for the MT sites of this study. Black squares indicate xy and red triangles yx component. 

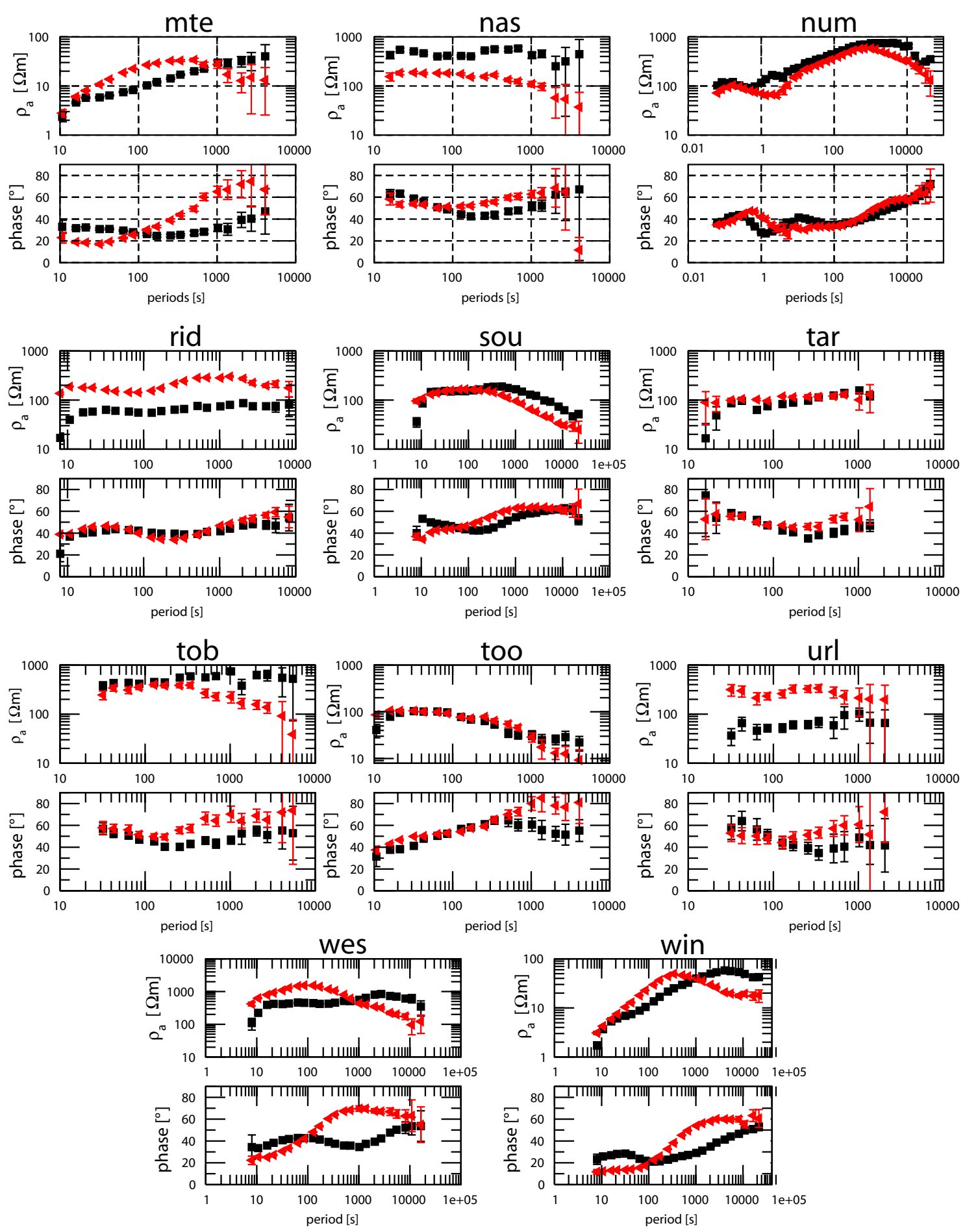

Figure A.4: Unrotated apparent resistivity and phase curves for the MT sites of this study. Black squares indicate xy and red triangles yx component. 


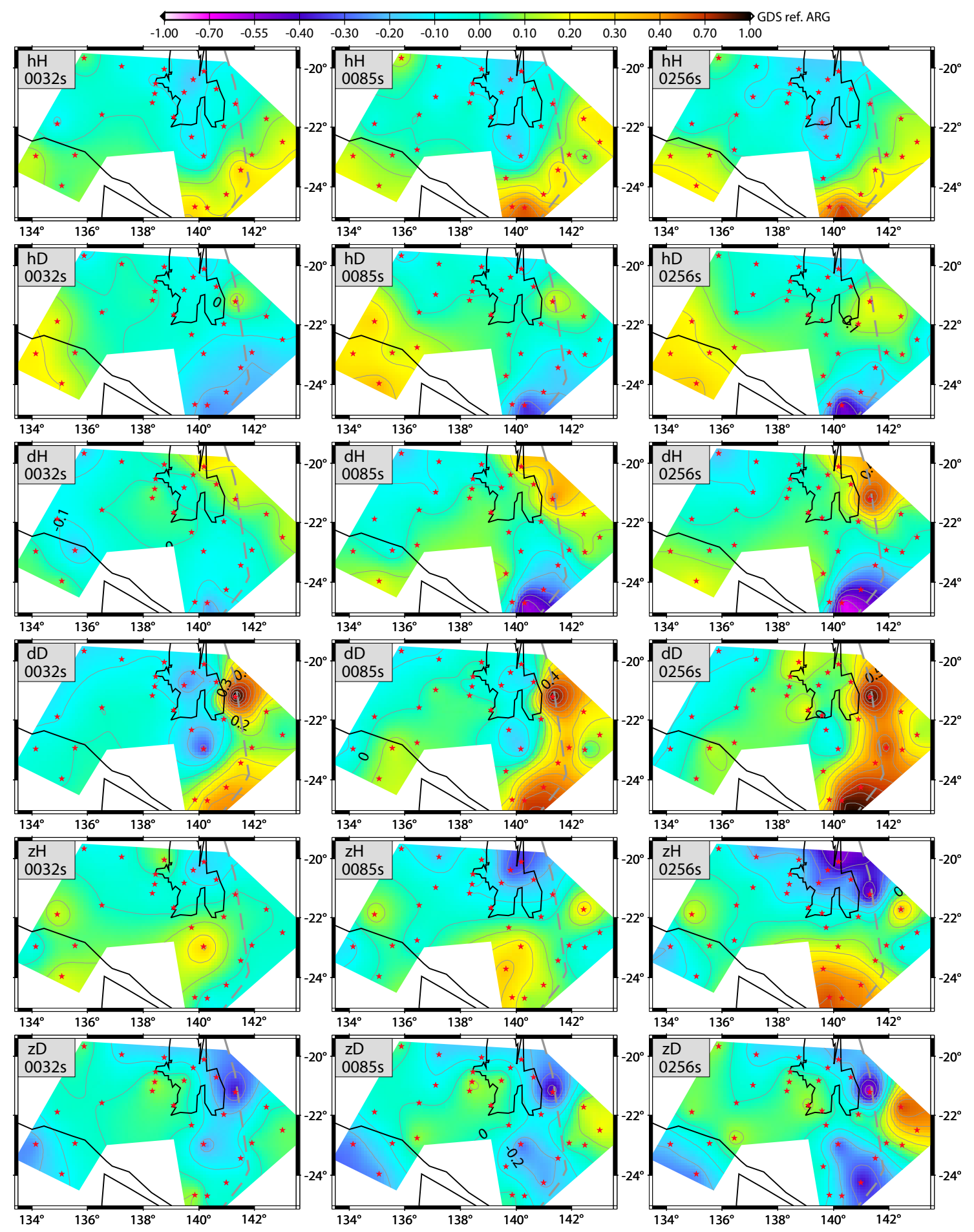

Figure A.5: Real parts of the perturbation tensor with site ARG as reference site 

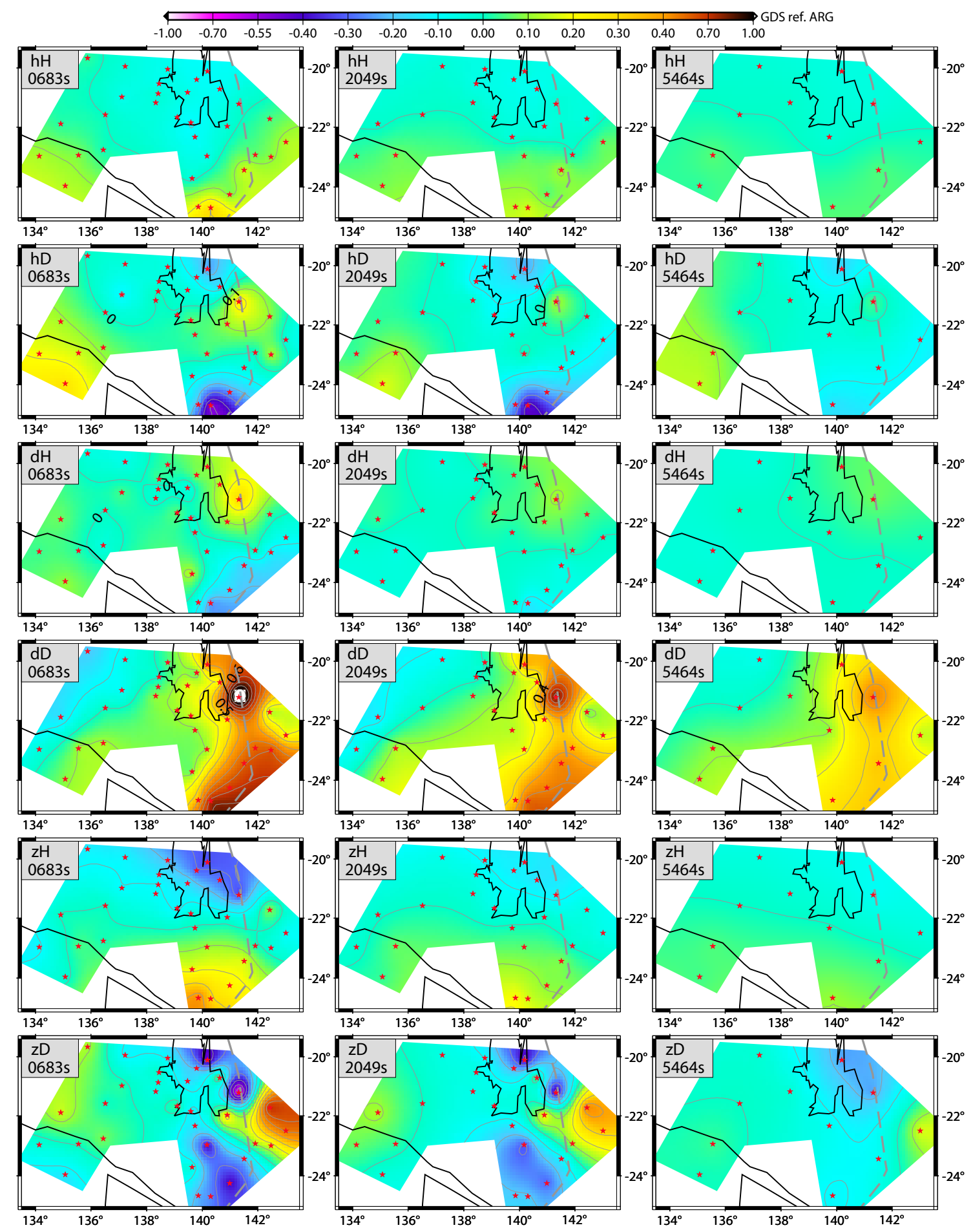

Figure A.6: Real parts of the perturbation tensor with site ARG as reference site 


\section{B Programs}

This chapter introduces some of the Fortran programs that have been written or modified for this thesis. While the most important features of the programs will be discussed this appendix is not meant to be a complete documentation of these programs. More information can usually be found as descriptions when running the programs or in the source code. All programs have been written in Fortran 77 with some extensions of the GNU Fortran compiler (gcc 4.0.2). Whenever MT or GDS field data are processed Göttingen type .mtt/.gdt transfer function file formats are used (ASCII files, see Leibecker (2000) for more information). Time series data is saved in the binary goe $^{1}$ format.

\section{B.1 Programs for display purposes}

\section{B.1.1 emdisp}

The program emdisp was written by Leibecker (2000) and extended by Gatzemeier (2002) and Moorkamp (2003). It reads Göttingen type MT and GDS data (.mtt/.gdt format, see e.g. Leibecker (2000)) and produces data files for frequency dependent data for one station (that can e.g. be plotted with the Linux program xmGrace,(Grace Development Team, 2005)) or files for map plots of certain MT or GDS parameters that can be plotted with the mapping tool GMT (Wessel \& Smith, 1998). While up to 512 files can be loaded (with the help of a station file containing filenames and coordinates) all files loaded at the same time must contain the same frequencies. The following calculations are included in the current version emdisp 1.3.2 (" +" as item symbol indicates new features, $[\mathrm{F}]$ frequency dependent and $[\mathrm{M}]$ mapplot results):

\section{MT measures (selection)}

- apparent resistivity and phase and impedance tensor elements [F,M] (Cagnard, 1953)

- Schmucker (1987) $\rho^{*}-z^{*}$ parameters $[\mathrm{F}]$

\footnotetext{
${ }^{1}$ The goe format is a binary (direct access) format. The record length is 256 bytes. The first 4 records (1024 bytes) contain header information including runtime, site-name, sampling rate and numbers of records in the file. The following records contain all data for the first saved component followed by the data of the second component (starting with a new record) and so on. More information can be found in the RAP data-logger manual (Steveling, 2000).
} 
- Swift (1967) and Bahr (1988) skew and rotation angles including a multi frequency version of Bahr (1988) decomposition [F,M]

+ Groom \& Bailey (1989) decomposition (inc. multi-frequency estimation) $[\mathrm{F}]$

- local induction arrows Wiese (1962) [F]

+ Chave \& Smith (1994) (experimental version) and Smith (1997) decompositions (inc. multi-frequency estimation) $[\mathrm{F}]$

+ Becken \& Burkhardt (2004) decomposition [F]

+ Berdichevsky [M,F] and Weaver et al. (2000) invariants [F]

+ Mohr circles (Lilley, 1998a,b) [F]

- phase ellipses $[\mathrm{M}]$

+ inductive strength (Lezaeta \& Haak, 2003) [M]

+ output for REBOCC 2D inversion (Siripunvaraporn \& Egbert, 2000) (site locations have to be modified by hand)

- output of a rotated .mtt file

\section{GDS measures (selection)}

- perturbation tensor elements $[\mathrm{F}, \mathrm{M}]$

- perturbation arrows $[\mathrm{F}, \mathrm{M}]$

- normal induction arrows $[\mathrm{M}]$

+ GDS strike angles (2 different versions) [F,M]

+ Mohr circles $[\mathrm{F}]$

All parameters can be evaluated for any coordinate system and all frequencies or rotation angle files can be read to rotate the different stations and frequencies separately.

\section{B.1.2 d3togmt}

d3togmt reads a 3D forward model file for the modeling code of d3fwd/d3_to_mt ${ }^{2}$ (Mackie \& Booker, 1999) to (i) create a station file for use with emdisp (ii) create data files that can be plotted with GMT (Wessel \& Smith, 1998) or (iii) creates

${ }^{2} \mathrm{~d} 3 \_$to_mt was modified to produce .gdt output of GDS data. For better handling of the modeling results a .mto and .gds with MT/GDS tensor element data is created that can be read by d3togmt 
resistivity layer data files for selected layers of the model file that can be plotted with GMT. The parameter that can be plotted include MT apparent resistivity, phase, local induction arrows, Bahr (1988) decomposition angles and GDS perturbation tensor elements.

\section{B.2 Data handling programs}

\section{B.2.1 gdsswap}

gdsswap can be used to change the reference station of GDS data (.gdt format). This is useful when the present reference site is located on a magnetic anomaly that affects the GDS transfer functions at all other locations or when different arrays with one common station which is not the reference station shall be combined. The following two functions are implemented:

- change the reference and field site in one .gdt file

- change of the reference site for a set of sites in a gdsswap.cfg file

The output are gdt files with GDS transfer functions with respect to the new reference site. Note that when multiple files are transferred to a new reference the quality of the transfer functions between old and new reference site is essential for the data quality of all converted .gdt files.

\section{B.2.2 3layer1d}

3layer1d is a simple inversion program for MT data. The data of a .mtt file with MT impedance tensor data is fitted by a three layer model. The model consists of a top layer, an anisotropic middle layer and a homogenous half-space. This model has six parameters, the two layer thicknesses and 4 resistivities $\left(\rho_{1}, \rho_{2, x}, \rho_{2, y}\right.$ and $\left.\rho_{3}\right)$. Models for all parameter combinations (user defined model space size and step size for each parameter) are calculated to find the best fitting model. Output parameters are the final model parameters and the misfit.

\section{B.2.3 multilayer1d}

multilayer1d is a 1D forward modeling program that allows for anisotropy in every layer. An infinite number of layers can be used. The main limitation of this program is that the anisotropy direction can only be in one the main polarization directions. Therefore arbitrarily varying directions from layer to layer are not possible.

\section{B.2.4 tipper2mt}

The program tipper2mt reads local induction arrow array data to estimate the corresponding GDS and MT transfer functions following the algorithm of Becken \& 


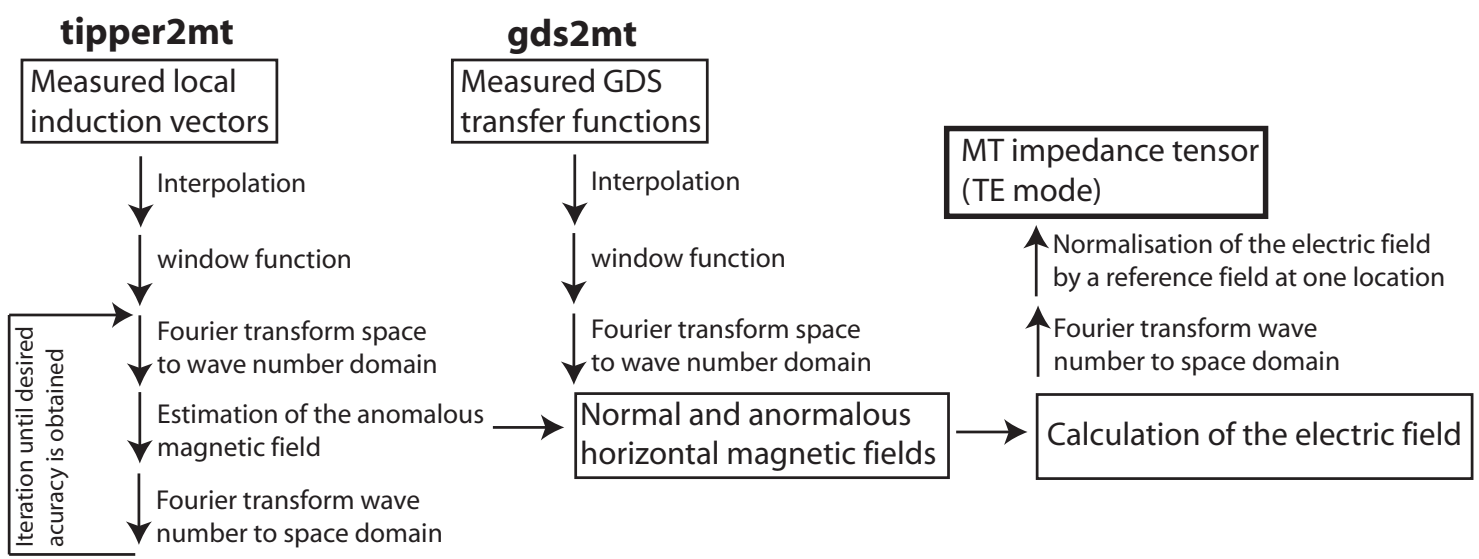

Figure B.1: Scheme of the programs tipper2mt and gds2mt

Pedersen (2003) that is described in section 2.5. As the electromagnetic field components are estimated in the wavenumber domain this approach is only applicable for large arrays with sufficient data coverage. The input data has to be in the system independent netCDF format that is e.g. created by the program surface of the GMT (Wessel \& Smith, 1998) package. The best way to apply tipper2mt on MT data is to use the map-plot function of the vertical magnetic transfer functions in emdisp (Appendix B.1.1) to create a .dat file with the data of all stations for each period. In a second step the GMT programs blockmean and surface can be used to convert the data to a regular grid in the netCDF format. This output .grd file can be read by tipper2mt. In order to calculate the MT transfer function the amplitude of the electric field has to be given. This is done by providing apparent resistivity data at one grid point. Figure B.1 illustrates the different steps done by the program.

\section{gds2tipper and gds2mt}

The program gds2tipper is a modified version of tipper2mt. It reads regularly gridded horizontal magnetic transfer functions (8 files: 4 real and 4 imaginary parts) to calculate corresponding local induction arrows using the Hilbert transform. As the anomalous magnetic field is given in the GDS transfer functions the iterative procedure in tipper2mt could be replaced by a simple calculation in the wave number domain. In addition magnetotelluric impedances can be calculated from the GDS transfer functions using a given impedance at one location as reference.

gds2mt is uses the horizontal GDS transfer functions to estimate the MT impedance tensor elements using the same approach as gds2tipper and tipper2mt.

\section{B.2.5 Other programs}

\section{intm2sqq}

intm2sqq reads intermagnet (www.intermagnet.org) magnetic observatory data (in IMFV1_22 format) and converts them to the Göttingen type .sqq format with hourly 
mean values of the magnetic field. These .sqq files can be read with the program sqplay (see e.g. Soyer (1998)) to calculate geomagnetic transfer functions of the $S_{q}$-current system.

\section{goe2asc}

goe2asc converts Göttingen type time series data (.goe) to ASCII format (.asc) that can be read with the processing software of Egbert \& Booker (1986); Egbert (1997). With the program zrr2mtt the resulting .zss/.zrr/.zmm (single site/remote reference/multi-site) transfer functions can be converted to the .mtt format.

\section{aspconv}

aspconv converts magnetic observatory data from Geoscience Australia magnetic observatories into the goe time series format. This includes linear time interpolation if the sampling changes with time.

\section{mtt3dinv}

mtt3dinv converts .mtt files and site locations in an input station file to a data file that can be used with the 3D MT inversion wsinv3dmt by Siripunvaraporn et al. (2005a). This feature was not included in emdisp as different numbers of frequencies should be allowed for the different files. As wsinv3dmt requires the same number of frequencies for all sites data gaps at some sites are filled with the data of the nearest available frequency. To take account for errors due to this extension of the data the errors of the impedances are set to the impedance values. Furthermore a conversion from geographic coordinates to locations in the $\mathrm{x}-\mathrm{y}$ plane and a selection of output frequencies are included.

\section{3dinv2mtt}

3dinv2mtt converts the model responses of the 3D inversion to .mtt files. A station file with file names and coordinates that can be used with GMT is created as well.

\section{3dinv2gmt}

3dinv2gmt converts wsinv3dmt model files to .dat files that can be plotted with GMT. Vertical and horizontal slices can be produced. As similar program inv2gmt exists to convert 2D model files of the REBOCC inversion (Siripunvaraporn \& Egbert, 2000) to .dat files. 


\section{GDS to MT transformations for 2D background structures}

Chapter 2.5 introduced an algorithm to convert magnetic to magnetotelluric transfer functions (Becken \& Pedersen, 2003). This algorithm assumes uniform background structures at the data array borders. Non-uniform background structures lead to non-vanishing average magnetic transfer functions (Chapter 5 ). Therefore additional terms have to be added to equation 2.58. This follows directly from the Fourier transformation:

$$
\tilde{F}\left(k_{x}\right)=\int_{-\infty}^{\infty} f(x) e^{-i k_{x} x} \mathrm{dx}
$$

(For simplicity only the continuous one-dimensional Fourier transform is used but generalizations to discrete multidimensional transformations are trivial.) For a simple $2 \mathrm{D}$ model, e.g. two quarter spaces, the resistivity changes in one direction only. For structures striking in $\mathrm{x}$-direction only a vertical magnetic field induced by a magnetic field in y-direction exist and the z-component of equation 2.57 simplifies to $\frac{\partial E_{x}}{\partial y}=i \omega B_{z} \Rightarrow E_{x}(y)=-i \omega \int_{0}^{y} B_{z}\left(y^{\prime}\right)$ dy'. With the definition of the Fourier transform (C.1) follows:

$$
\widetilde{E_{x}}\left(k_{y}\right)=i \omega \int_{-\infty}^{\infty} e^{-i k_{x} x}\left(\int_{0}^{y} B_{z}\left(y^{\prime}\right) \mathrm{dy} y^{\prime}\right) \mathrm{dy} .
$$

Partial integration leads to

$$
\widetilde{E_{x}}\left(k_{y}\right)=i \omega\left(\left[\frac{1}{-i k_{y}} e^{-i k_{x} x} \int_{0}^{y} B_{z}\left(y^{\prime}\right) \mathrm{dy}^{\prime}\right]_{-\infty}^{\infty}-\int_{-\infty}^{\infty} \frac{1}{-i k_{y}} e^{-i k_{x} x} B_{z}(y) \mathrm{dy}\right)
$$

While as applied in Becken \& Pedersen's algorithm the second term is $1 /\left(-i k_{y}\right)$ times the Fourier transform of $B_{z}$ the existence of the first term depends on the constant component of $B_{z}$. For 1D background structures the constant component of $B_{z}$ vanishes for arbitrary conductivity structures in the central part. For multidimensional background structures however a constant component exists and the first term in equation (C.3) has to be considered. In case of known 2D structures the problem can be overcome by space-domain integration following equation (5.1).

The effect of non-homogenous background structures on the conversion algorithm can be illustrated by a quarter-space model. Figure C.1 shows the E-Polarization (xy-component) MT apparent resistivities and phases as modeled, obtained from the standard conversion algorithm (1D background and following equation (5.1) (2D background). As expected a good agreement can be seen between the modeled 

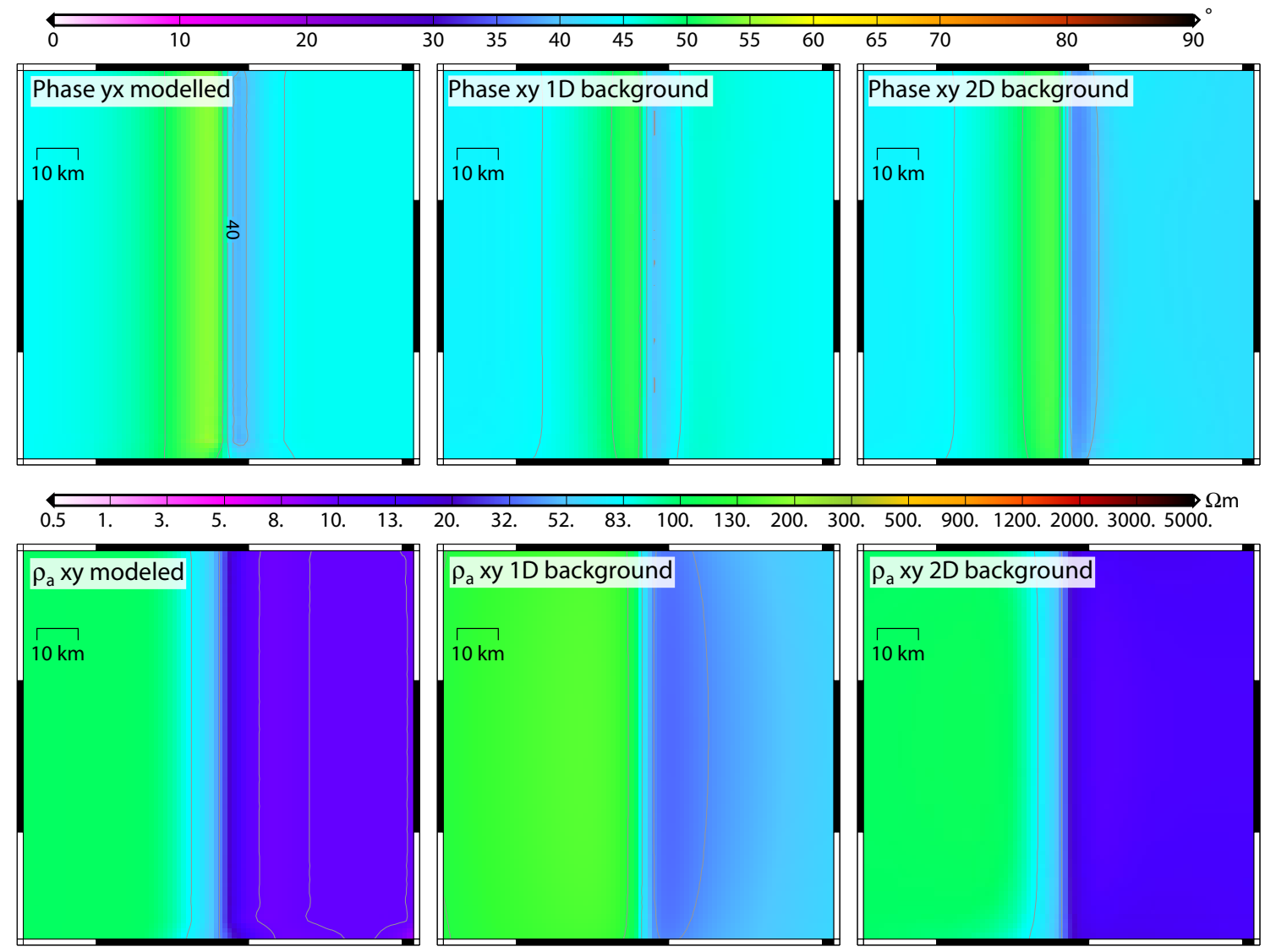

Figure C.1: Apparent resistivities and phase values of the xy-component of the MT impedance tensor of a quarter-space model and a period of 8 seconds. The western region has a resistivity of $100 \Omega m$ while the eastern part has $10 \Omega \mathrm{m}$. The modeled results are compared to those converted from the vertical magnetic transfer functions assuming 1D or $2 \mathrm{D}$ background structures.

results and those correctly assuming a 2D background structure. The results of the conversion assuming a 1D background show correct values on the site of the chosen electric field reference site but are wrong east of the resistivity contrast. This is related to the non-vanishing constant part of the vertical magnetic transfer functions. The minor differences between modeled and 2D background results are related to discretization errors. A similar but more detailed space domain treatment of magnetic to magnetotelluric data conversions in 2D environments is given by Gharibi \& Pedersen (1999).

An extension of the conversion algorithm to arbitrary background structures is complicated by the mixture of the horizontal derivatives of the electric field in the z-component of equation (5.1). Therefore the present approach does not allow conversions of magnetic to magnetotelluric data without detailed knowledge about conductivity gradients at the array boundaries. 


\section{Modeling details}

The final forward model (Figure 6.1) for the Australian dataset was presented in Chapter 6. This Chapter gives some more details about the forward modeling and the final model.

The modeling code of Mackie \& Booker (1999) handles the boundary conditions at the model boarders by assuming continuous $2 \mathrm{D}$ structures. It is nevertheless recommended to extend the resistivity models to dimensions larger than the area of interest to avoid undesired influences of the boundary conditions. For the final model of the Australian dataset the extension of the model space leads to final model dimensions of more than $1510 \mathrm{~km} \times 1730 \mathrm{~km} \times 200 \mathrm{~km}$. The lateral gridding of the model is plotted in Figure D.1. Each dot represents a model site. The lateral grid site in the area of interest (gray box in the Figure) is $6250 \times 6250 \mathrm{~m}$ with increasing grid sizes towards the boundaries.

The code computes MT and GDS transfer functions for each model cell. Therefore the site spacing of such over-parameterized models is significantly smaller than the measured site spacing. When the model responses are plotted this leads to much smaller resolvable model structures than those resolvable by the field data. Therefore interpolations between model sites that match measured field site locations have been used for the model response plots (Figures 6.2 and 6.3). In order to demonstrate the difference in resolution Figure D.2 compares results of the phase response (xy-component) interpolated between the 36 model sites representing the field measurements to the response functions of all 34081 model sites. The most significant differences are visible at the model boundaries where extrapolations in areas that are not covered by measuring sites lead to wrong results (Figure D.2a). This is particularly obvious in the north-eastern part of the array. In addition the full resolution results clearly identify the resistivity contrasts in the model while they are smoothed by the interpolation of field site data coverage. This effect is even bigger for the apparent resistivities that are particularly effected by distortion effects related to shallow conductivity contrasts in the model.

Figure D.3 shows the real parts of all GDS perturbation tensor elements for three periods. The results are interpolated between measured field sites to simplify comparisons to the measured results of Appendix A.3 Figures A.5+A.6. 


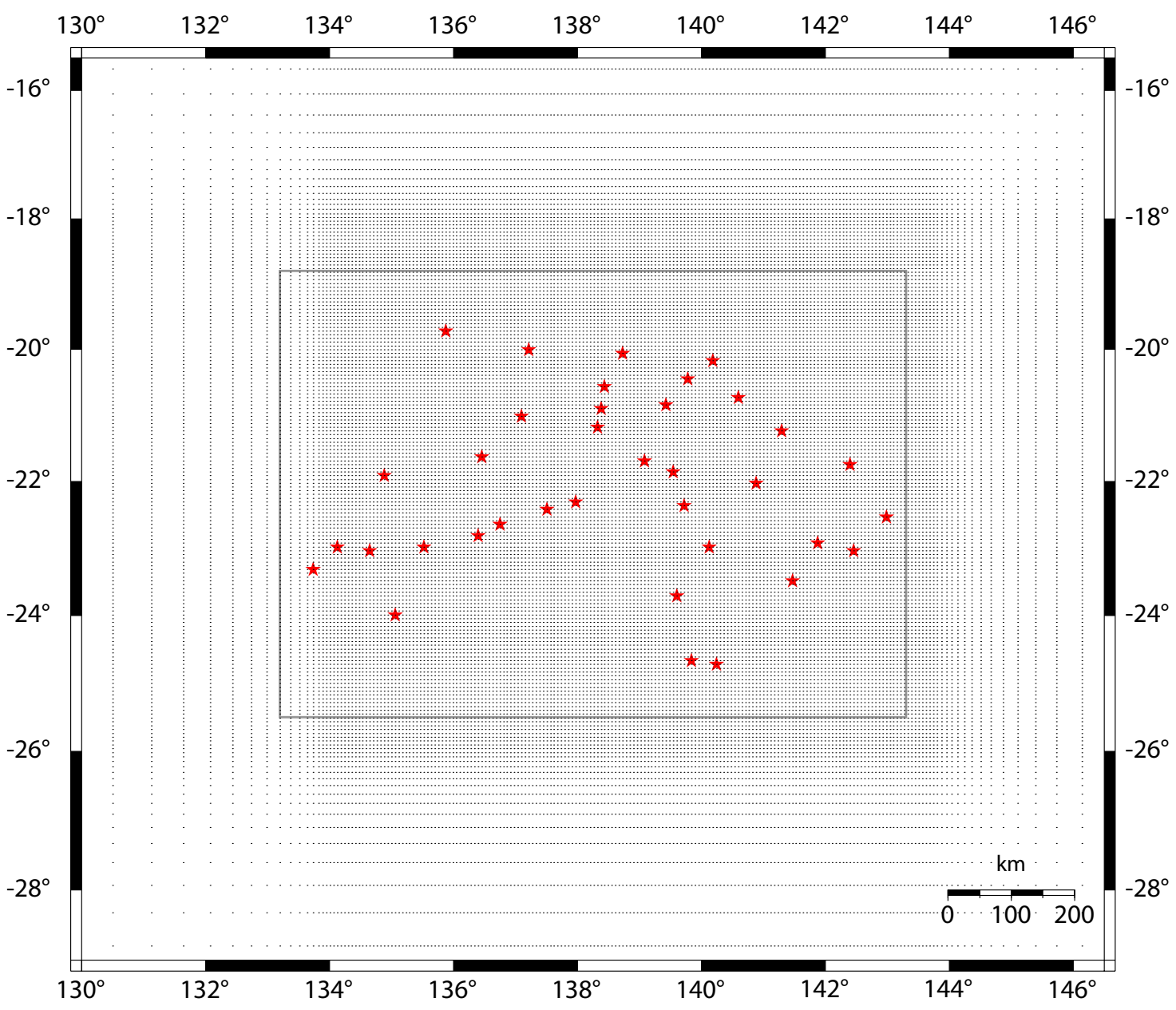

Figure D.1: Grid of the final 3D forward model for the Australian dataset. Each dot represents a model site. The gray box shows the area of interest used for the model response function plots. The red Stars indicate field sites.

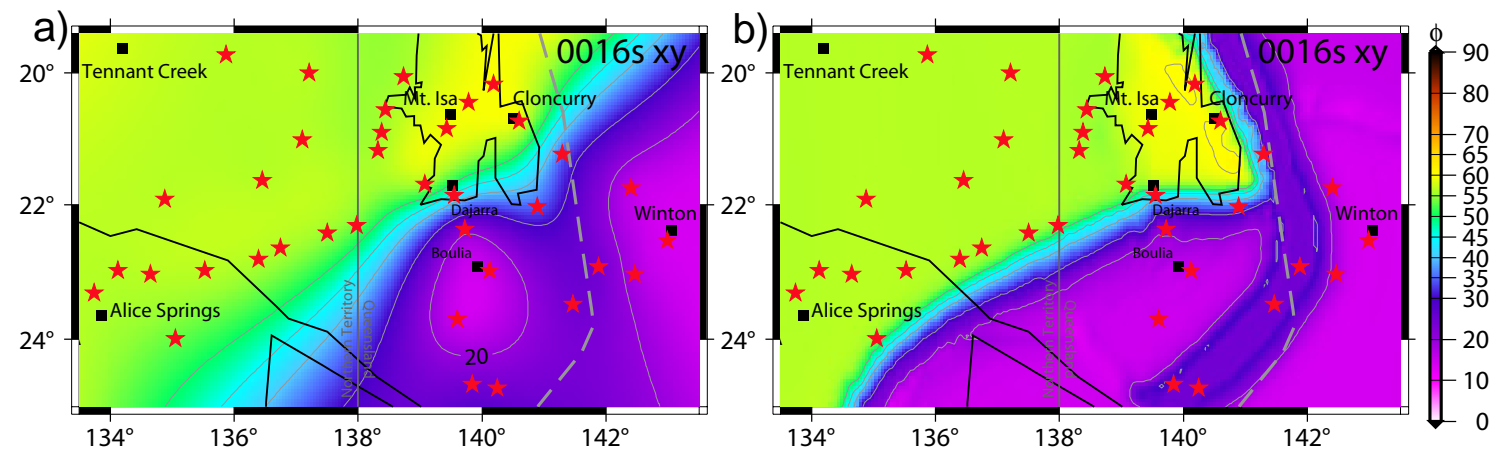

Figure D.2: Comparison of the model responses for the final 3D forward model of the Australian dataset as (a) interpolated between model sites at measured site locations (36 sites) and (b) using all 34081 model sites. 


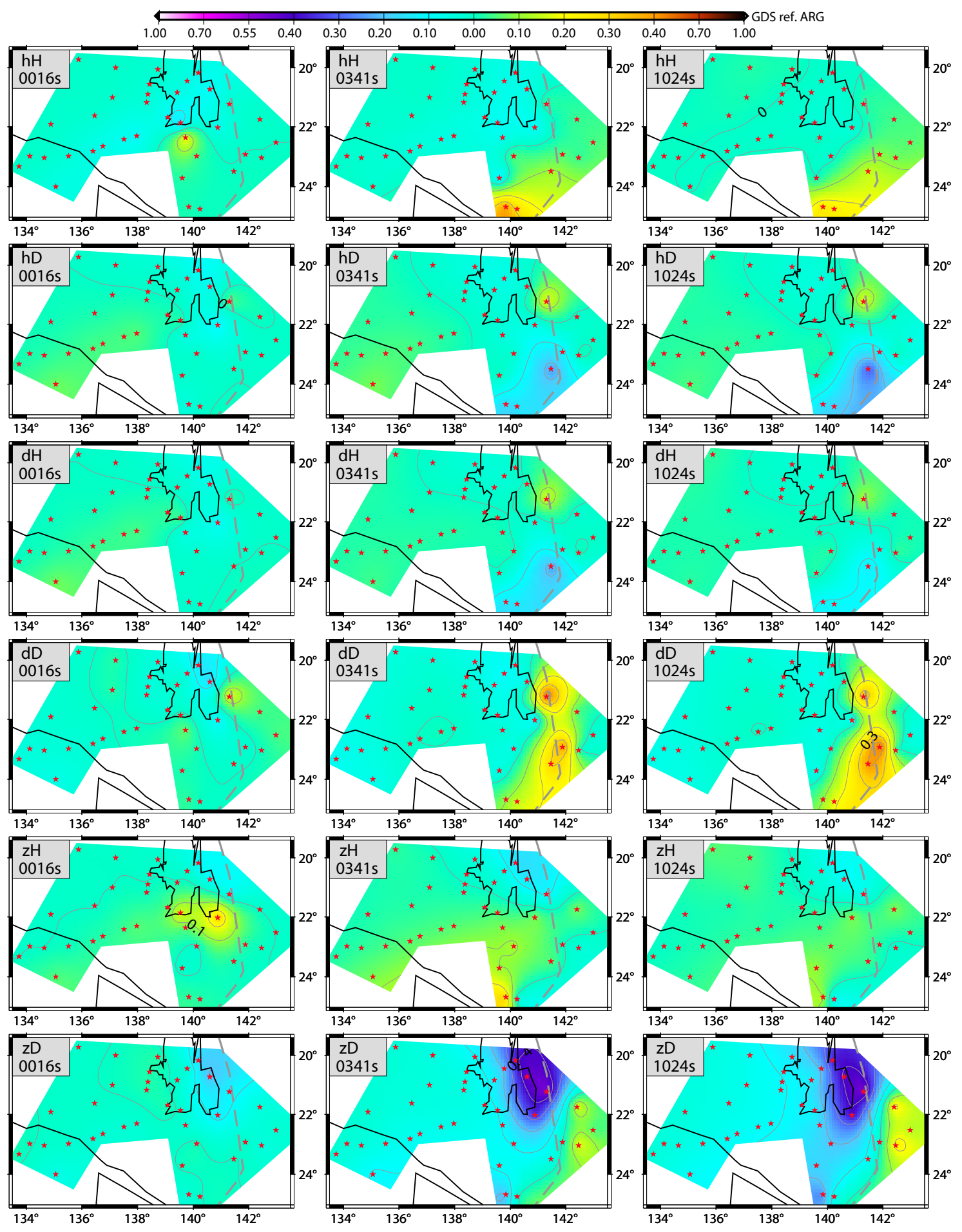

Figure D.3: Real parts of the perturbation tensor elements of the final 3D forward model. The red stars indicate the field site location used for the interpolation of this plot. 
D Modeling details 


\section{Bibliography}

Agarwal A.K.; Weaver J.T., 2000. Magnetic distortion of the magnetotelluric tensor: a numerical study. Earth Planets Space, 52, 347-353.

Bahr K., 1988. Interpretation of the magnetotelluric impedance tensor: regional induction and local telluric distortion. Journal of Geophysics, 62, 119-127.

Bahr K., 1991. Geological noise in magnetotelluric data: A classification of distortion types. PEPI, 66, 24-38.

Bahr K., 1997. Electrical anisotropy from and conductivity distribution functions of fractal random networks and of the crust: The scale effect of connectivity. GJI, 130, 649-660.

Bahr K., 2000. Percolation in the crust derived from distortion of electric fields. Geophysical Research Letters, 27, 1049-1052.

Bahr K.; Simpson F., 2002. Electrical anisotropy below slow- and fast-moving plates: Paleoflow in the upper mantle? Science, 295, 1270-1272.

Balasis G.; Banks R.J.; Whaler K., 1997. Comment on 'Understanding telluric distortion matrices' by J. T. Smith. Geophysical Journal International, 129, 472473.

Becken M.; Burkhardt H., 2004. An ellipticity criterion in magnetotelluric tensor analysis. Geophysical Journal International, 159, 69-82.

Becken M.; Pedersen L., 2003. Transformation of VLF anomaly maps into apparent resistivity and phase. Geophysics, 68(2), 497-505.

Berdichevsky M., 1999. Marginal notes on magnetotellurics. Surveys in Geophysics, 20, 341-375.

Berdichevsky M.; Dmitriev V.; Pozdnjakova E., 1998. On two-dimensional interpretation of magnetotelluric soundings. Geophysical Journal International, 133, $585-606$.

Berdichevsky M.; Zhdanov M., 1984. Advanced theory of deep geomagnetic sounding. Elsevier.

Betts P., 1999. Paleoproterozoic mid-basin inversion in the northern Mt Isa terrane, Queensland. Australian Journal of Earth Sciences, 46, 735-748. 
Betts P.; Giles D.; Lister G.; Frick L., 2002. Evolution of the Australian lithosphere. Australian Journal of Earth Sciences, 49, 661-695.

Betts P.; Giles D.; Mark G.; Lister G.; Goleby B.; Ailleres L., 2006. Synthesis of the Proterozoic evolution of the Mt Isa Inlier. Australian Journal of Earth Sciences, $53,187-211$.

Bibby H.; Cladwell T.; Brown C., 2005. Determinable and non-determinable parameters of galvanic distortion in magnetotellurics. Geophysical Journal International, 163, 915-930.

Bierlein F.; Betts P., 2004. The proterozoic Mount Isa fault zone, northeastern Australia: is it really a ca. 1.9 Ga terrane-bounding suture? Earth and Planetary Science Letters, 225, 279-294.

Boerner D.E.; Kurtz R.; Craven J.; Ross G.; Jones F.; Davis W., 1999. Electrical conductivity in the Precambrian lithosphere of western Canada. Science, 283, 668-670.

Boger S.; Hansen D., 2004. Metamorphic evolution of the Georgetown Inlier, northeast Queensland, Australia; evidence for an accreted Paleoproterozoic terrane? J. metamorphic Geol., 22, 511-527.

Cagnard L., 1953. Basic theory of the magneto-telluric method of geophysical prospecting. Geophysics, 18, 605-635.

Campbell W.; Barton C.; Chamalaun F.; Welsh W., 1998. Quiet-day ionospheric currents and their application to upper mantle conductivity in Australia. Earth Planets Space, 50, 347-360.

Campbell W.H., 1997. Introduction to geomagnetic fields. Cambridge University Press.

Chamalaun F.; Barton C., 1993. The large-scale electrical conductivity structure of Australia. J. Geomag. Geoelectr., 45, 1209-1212.

Chamalaun F.; Lilley F.; Wang L., 1999. Mapping the Carpentaria conductivity anomaly in northern Australia. PEPI, 116, 105-115.

Chave A.; Smith T., 1994. On electric and magnetic galvanic distortion tensor decompositions. Journal of Geophysical Research, pages 4669-4682.

Clitheroe G., 1999. Receiver based studies of the australian continent. Ph.D. thesis, Research School of Earth Sciences, Australian National University.

Clitheroe G.; Gudmundsson O.; Kennett B., 2000a. Sedimentary and upper crustal structure of Australia from receiver functions. Australian Journal of Earth Sciences, 47, 209-216. 
Clitheroe G.; Gudmundsson O.; Kennett B.L.N., 2000b. The crustal thickness of Australia. Journal of Geophysical Research, 105, 13697-13713.

Collins C.; Drummond B.; Nicoll M., 2003. Crustal thickness patterns in the Australian continent. In R. Hillis; R. Müller, editors, Evolution and dynamics of the Australian plate, volume 22 of Geological Society of Australia Special Publication, pages $121-128$.

Constable S.; Parker R.; Constable C., 1987. Occam's inversion: A parctical algorithm for generating smooth models from electromagnetic sounding data. Geophysics, 52(3), 289-300.

Crawford A.; Meffre S.; Symonds P., 2003. 120 to 0 Ma tectonic evolution of the southwest pacific and analogous geological evolution of the 600 to 220 ma tasman fold belt system. In R. Hillis; R. Müller, editors, Evolution and dynamics of the Australian plate, volume 22 of Geological Society of Australia Special Publication, pages $383-403$.

Cull J., 1982. Magnetotelluric profiles in the McArthur basin of northern Australia. BMR Journal of Australian Geology and Geophysics, 7, 275-286.

Debayle E.; Kennett B., 2000. The australian continental upper mantle: Structure and deformation inferred from surface waves. Journal of Geophysical Research, 105(B11), 25423-25450.

Debayle E.; Kennett B., 2003. Surface-wave studies of the Australian region. In R. Hillis; R. Müller, editors, Evolution and dynamics of the Australian plate, volume 22 of Geological Society of Australia Special Publication, pages 25-40.

deGroot Hedlin C.; Constable S., 1990. Occam's inversion to generate smooth, twodimensional models from magnetotelluric data. Geophysics, 55(12), 1613-1624.

deGroot Hedlin C.; Constable S., 2004. Inversion of magnetotelluric data for 2D structure with sharp resistivity contrasts. Geophysics, 69(1), 78-86.

Direen N.; Crawford A., 2003. The Tasman Line: Where is it, what is it and is it Australia's Rodinian breakup boundary? AJES, 50, 491-502.

Drummond B.; Goleby B.; Goncharov A.; Wyborn L.; Collins C.; MacCready T., 1998. Crustal-scale structures in the proterozoic Mount Isa inlier of north Australia: their seismic response and influence on mineralisation. Tectonophysics, $288,43-56$.

Egbert G., 1990. Comments on 'concerning dispersion relations for the magnetotelluric impedance tensor' by E. Yee and K. V. Paulson. Geophysical Journal International, 102, 1-8. 
Egbert G.; Booker J.R., 1986. Robust estimation of geomagnetic transfer functions. Geophysical Journal of the Royal astronomical Society, 87, 173-194.

Egbert G.D., 1997. Robust multiple-station magnetotelluric data processing. Geophysical Journal International, 130, 475-496.

Eisel M.; Haak V., 1999. Macro-anisotropy of the electrical conductivity of the crust: a magnetotelluric study of the german continental deep drilling site (KTB). Geophysical Journal International, 136, 109-122.

Ewers G.; Evans N.; Kilgour B., 2001. MINLOC mineral localities database. Digital Dataset, http://www.ga.gov.au. Geoscience Australia.

Fischer G.; Schnegg P.A., 1993. The magnetotelluric dispersion relations over 2-D structures. Geophysical Journal International, 115, 1119-1123.

Fishwick S.; Kennett B.; Reading A., 2005. Contrasts in lithospheric structure within the Australian craton - insights from surface wave tomography. Earth and Planetary Science Letters, 231, 163-176.

Fluche B., 1983. Erdmagnetische Tiefensondierung und Magnetotelluric in der Hessischen Senke (in German). Master's thesis, Institut für Geophysik, Universität Göttingen.

GABCC, 1998. Great artesian basin resource study summary. http://www.gab.org.au/. Great Artesian Basin Consultative Council.

GABCC, 2000. Great arteasian basin; strategic management plan. The Great Artesian Basin Consultative Council.

Gallardo L.; Meju M., 1993. Characterization of heterogeneous near-surface materials by joint 2D inversion of de resistivity and seismic data. GRL, 30(13), doi:10.1029/2003GL017370.

Gamble T.D.; Goubau W.M.; Clarke J., 1979. Magnetotellurics with a remote magnetic reference. Geophysics, 44, 53-68.

Gatzemeier A., 2002. Elektrische Anisotropie durch ausgerichtete Olivinkristalle im oberen Mantel in Mitteleuropa: Magnetotellurische Array-Messungen und ein Ansatz zum Vergleich mit seismischer Anisotropie. Ph.D. thesis, Universität Göttingen.

Gatzemeier A.; Moorkamp M., 2005. 3-D modelling of electical anisotropy from electromagnetic array data: hypothesis testing for different upper mantle conduction mechanisms. Physics of the Earth and Planetary Interiors, 149, 225-242.

Gharibi M.; Pedersen L., 1999. Transformation of VLF data into apparent resistivities and phases. Geophysics, 64(5), 1393-1402. 
Giles D.; Betts P.; Ailleres L.; Hulscher B.; Hough M.; Lister G., 2006. Evolution of the Isan orogeny at the southeastern margin of the Mt Isa inlier. Australian Journal of Earth Sciences, 53, 91-108.

Giles D.; Betts P.; Lister G., 2002. Far-field continental backarc setting for the 1.80-1.67 Ga basins of northeastern Australia. Geology, 30(9), 823-826.

Giles D.; Betts P.; Lister G., 2004. 1.8-1.5-Ga links between the north and south Australian cratons and the early-middle proterozoic configuration of Australia. Tectonophysics, 380, 27-41.

Goes S.; Simons F.; Yoshizawa K., 2005. Seismic constraints on temperature of the australian uppermost mantle. Earth and Planetary Science Letters, 236, 227-237.

Grace Development Team G., 2005. 2d plotting tool for the $\mathrm{x}$ window system. http://plasma-gate.weizmann.ac.il/Grace/.

Griffin W.; Belousova E.; Walters S.; O’Reilley S., 2006. Archean and Proterozoic crustal evolution in the eastern succession of the Mt Isa district, Australia: U-Pb and Hf-isotope studies of detrital zircons. AJES, 53, 125-149.

Groom R.; Bahr K., 1992. Corrections for near surface effects: Decomposition of the magnetotelluric impedance tensor and scaling corrections for regional resistivities: a tutorial. Surveys in Geophysics, 13, 341-379.

Groom R.; Bailey R., 1989. Decomposition of magnetotelluric impedance tensors in the presence of local three-dimensional galvanic distortion. Journal of Geophysical Research, 94, 1913-1925.

Gunn P., 1997. Regional magnetic and gravity responses of extensional sedimentary basins. Journal of Australian Geology and Geophysics, 17(2), 115-131.

Gunn P.; Dentith M., 1997. Magnetic responses assocoated with mineral deposits. Journal of Australian Geology and Geophysics, 17(2), 145-158.

Gunn P.; Milligan P.; Mackey T.; Liu S.; Murray A.; Maidment D.; Haren R., 1997. Geophysical mapping using the national airborne and gravity datasets; an example focusing on Broken Hill. Journal of Australian Geology and Geophysics, $17,127-136$.

Habashy T.; Groom R. ans Spies B., 1993. Beyond the Born and Rytov approximations: A nonlinear approach to electromagnetic sounding. Journal of Geophysical Research, 98, 1759-1775.

Habermehl R.; Pestov I., 2002. Geothermal resources of the Great Artesian Basin, Australia. GHC Bulletin, pages 20-26. 
Hanekop O., 2003. Zeitliche und räumliche Verzerrung von elektrischen Feldern in seismotektonisch aktiven Gebieten (in german). Master's thesis, Universität Göttingen.

Hanekop O.; Simpson F., 2006. Error propagation in electromagnetic transfer functions: What role for the magnetotelluric method in detecting earthquake precursors? GJI, 165, 763-774.

Heinson G.; White A., 2005. Electrical resistivity of the northern australian lithosphere: Crustal anisotropy or mantle heterogeneity? Earth and Planetary Science Letters, $232,157-170$.

Heintz M.; Kennett B., 2005. Continental scale shear wave splitting analysis: Investigation of seismic anisotropy underneath the australian continent. Earth and Planetary Science Letters, 236, 106-119.

Heise W.; Pous J., 2003. Anomalous phases exceeding $90^{\circ}$ in magnetotellurics: anisotropic model studies and a field example. Geophysical Journal International, 155, 308-318.

Hill D., 1951. Geology. In G. Mack, editor, Handbook of Queensland, pages 13-24. Australian Association for the Advancement of Science, Brisbane.

Hillis R.; Reynolds S., 2003. In situ stress field of Australia. In R. Hillis; R. Müller, editors, Evolution and Dynamics of the Australian Plate, volume 22 of Geological Society of Australia Special Publication, pages 49-58.

Jantos C., 1999. Audiomagnetotellurische Messungen mit der neuen Göttinger "Midband" Anlage in Bad Königshofen (in German). Master's thesis, Institut für Geophysik, Universität Göttingen.

Jones A., 1983. The problem of current channeling: A critical review. Geopysical Surveys, 6, 79-122.

Junge A., 1993. Induzierte erdelektrische Felder - neue Beobachtungen in Norddeutschland und im Bramwald. Habilitation, Universität Göttingen.

Karlstrom K.; Ahäll K.I.; Harlan S.; Williams M.; McLelland J.; Geissman J., 2001. Long-lived (1.8-1.0 Ga) convergent orogen in southern Laurentia, its extensions to Australia and Baltica, and implications for refining Rodinia. Precambrian Research, 111, 5-30.

Kellet R.; Mareschal M.; Kurtz R., 1992. A model of lower crustal electrical anisotropy for the Pontiac subprovince of the canadian shield. Geophysical Journal International, 111, 141-150.

Kennett B., 2003. Seismic structure in the mantle beneath Australia. In R. Hillis; R. Müller, editors, Evolution and dynamics of the Australian plate, volume 22 of Geological Society of Australia Special Publication, pages 7-23. 
Kennett B.L.N.; Fishwick S.; Reading A.M.; Rawlinson N., 2004. Contrasts in mantle structure beneath Australia: relation to tasman lines? Australian Journal of Earth Sciences, 51, 563-569.

Kohlstedt D.; Mackwell S., 1998. Diffusion of hydrogen and intrinsic point defects in olivine. Z. Phys. Chemie, 207, 147-162.

Larsen J., 1977. Removal of local surface conductivity effects from low frequency mantle response curves. Acta Geodaet., Geophys. et Montanist. Acad. Sci. Hung., $12,183-186$.

Leibecker J., 2000. Elektromagnetische Arraymessungen im Rheinischen Schiefergebirge: Modelle der elektrischen Leitfähigkeit der Erdkruste und des oberen Mantels mit Verbindungen zum Eifelvulkanismus (in German). Ph.D. thesis, Universität Göttingen. Http://webdoc.sub.gwdg.de/diss/2000/leibecker/.

Leibecker J.; Gatzemeier A.; Hönig M.; Kuras O.; Soyer W., 2002. Evidence of electrical anisotropic structures in the lower crust and the upper mantle beneath the Rhenish Shield. Earth and Planetary Science Letters, 202, 289-302.

Lezaeta P.; Haak V., 2003. Beyond magnetotelluric decomposition: Induction, current channeling and magnetotelluric phases over 90 degree. Journal of Geophysical Research, 108, EPM 4 1-20.

Li S.; Unsworth M.; Booker J.; Wei W.; Tan H.; Jones A., 2003. Partial melt or aqueous fluid in the mid-crust of southern Tibet? constraints from INDEPTH magnetotelluric data. Geophysical Journal International, 153, 289-304.

Lilley F., 1998a. Magnetotelluric tensor decomposition: Part 1, theory for a basic procedure. Geophysics, 63(6), 1885-1897.

Lilley F., 1998b. Magnetotelluric tensor decomposition: Part 2, examples of a basic procedure. Geophysics, 63(6), 1898-1907.

Lilley F.; Wang L.; Chamalaun F.; Ferguson I., 2003. The Carpentaria electrical conductivity anomaly, Queensland, as a major structure in the Australian plate. In R. Hillis; R. Müller, editors, Evolution and Dynamics of the Australian Plate, volume 22 of Geological Society of Australia Special Publication, pages 141-156.

Lilley F.; Woods D.; Sloane M., 1981. Electrical conductivity profiles and implications for the absence or presence of partial melting beneath central and southeast Australia. Physics of the Earth and Planetary Interiors, 25, 419-428.

MacCready T., 2006. Structural cross-section based on the Mt Isa deep seismic transect. Australian Journal of Earth Sciences, 53, 5-26.

MacCready T.; Goleby B.; Goncharov A.; Drummond B.; Lister G., 1998. A framework of overprinting orogens based on interpretation of the Mount Isa deep seismic transect. Economic Geology, 93, 1422-1434. 
Mackie R.; Booker J., 1999. Documentation for mtd3fwd and d3 to mt. Software manual.

Mackie R.; Madden T., 1993. Conjugate direction relaxation solutions for 3-D magnetotelluric modeling. Geophysics, 58(7), 1052-1057.

Mackie R.L.; Smith J.T.; Madden T.R., 1994. Three-dimensional electromagnetic modeling using finite differences equations: The magnetotelluric example. Radio Science, 29, 923-935.

Marcuello A.; Queralt P.; Ledo J., 2005. Applications of dispersion relations to the geomagnetic transfer function. Physics of the Earth and Planetary Interiors, 150, $85-91$.

Marti A.; Queralt P.; Jones A.; Ledo J., 2005. Improving Bahr's invariant parameters using the WAL approach. Geophysical Journal International, 163, 38-41.

Maxwell J., 1873. A Treatise on Electricity and Magnetism. Clarendon Press.

McLaren S.; Sandiford M.; Hand M.; Neumann N.; Wyborn L.; Bastrakova I., 2003. The hot southern continent: Heat flow and heat production in Austrtalian Proterozoic terranes. In R. Hillis; R. Müller, editors, Evolution and dynamics of the Australian plate, volume 22 of Geological Society of Australia Special Publication, pages $157-167$.

McLaren S.; Sandiford M.; Powell R., 2005. Contrasting styles of Proterozoic crustal evolution: A hot-plate tectonic model for the Australian terranes. Geology, 33(8), 673-676.

McNeice G.; Jones A., 2001. Multisite, multifrequency tensor decomposition of magnetotelluric data. Geophysics, 61(1), 158-173.

Moorkamp M., 2003. Elektrische Anisotropie im oberen Mantel (in German). Master's thesis, Institut für Geophysik, Universität Göttingen.

Moorkamp M.; Gatzemeier A., 2006. Electrical anisotropy in the upper mantle under Germany and the uplift of Fennoscandia. GJI (submitted).

Murray A., 2001. Gravity anomaly grid of the australian region. Digital Dataset. Http://www.ga.gov.au/general/technotes/20011023-32.jsp.

Myers J.; Shaw R.; Tyler I., 1996. Tectonic evolution of proterozoic Australia. Tectonics, 15(6), 1431-1446.

Nelson J., 1986. An alternate derivation of the three-dimensional Hilbert transform relations from first principles. Geophysics, 51(4), 1014-1015. 
Nover G.; Stoll J.; von der Gönna J., 2005. Promotion of graphite formation by tectonic stress - a labratory experiment. Geophysical Journal International, 160, 1059-1067.

Ogawa Y.; Uchida T., 1996. A two-dimensional magnetotelluric inversion assuming gaussian static shift. Geophysical Journal International, 126, 69-76.

Olsen N., 1998. The electrical conductivity of the mantle beneath Europe derived from C-responses from 3 to 720 hr. Geophysical Journal International, 133, 298308.

Parkinson W., 1959. Direction of rapid geomagnetic fluctuations. Geophysical Journal, 2, 1-14.

Pedersen L., 1989. Relations between horizontal and vertical gradients of potential fields. Geophysics, 54(5), 662-663.

Petkovic P.; Buchanan C., 2002. Australian bathymetry and topography grid (digital dataset). Canberra: Geoscience Australia. Http://www.ga.gov.au.

Petkovic P.; Milligan P., 2002. Magnetic anomaly grid of the australian region, 3.1. Digital Dataset. Http://www.ga.gov.au/general/technotes/20011023-32.jsp.

Reynolds J., 1997. An introduction to applied and environmental geophysics. John Wiley and Sons. ISBN 0-471-95555-8.

Reynolds S.; Mildren S.; Hillis R.; Meyer J.; Flottmann T., 2005. Maximum horizontal stress orientations in the Cooper basin, Australia: implications for plate-scale tectonics and local stress sources. Geophysical Journal International, 160, 331343.

Ritter P., 1996. Separation of local and regional information in geomagnetic response functions using hypothetical event analysis. Ph.D. thesis, University of Edinburgh, Dept. of Geology and Geophysics.

Ritter P.; Banks J., 1998. Separation of local and regional information in distorted gds response functions by hypothetical event analysis. Geophysical Journal International, 135, 923-942.

Roberts J.; Duba A.; Mathez E.; Shankland T.; Kinzler R., 1999. Carbon-enhanced electrical conductivity during fracture of rocks. Journal of Geophysical Research, 104, 737-747.

Rodi W.; Mackie R., 2001. Nonlinear conjugate gradients algorithm for 2-D magnetotelluric inversion. Geophysics, 66(1), 174-187.

Schmucker U., 1970. Anomalies of geomagnetic variations in the southwestern United States, volume 13. Bull. Scripps Institution of Oceanography. 
Schmucker U., 1987. Substitute conductors for electormagnetic respone estimates. Pure and Applied Geophysics, 125(2-3), 341-367.

Schmucker U.; Weidelt P., 1975. Electromagnetic induction in the Earth. Lecture notes Aarhus.

Selway K.; Heinson G.; Hand M., 2006. Electrical evidence of continental accretion: Steeply-dipping crustal-scale conductivity contrast. Geophysical Research Letters, 33, doi:10.1029/2005GL025328.

Siemon B., 1997. An interpretation technique for superimposed induction anomalies. Geophysical Journal International, 130, 73-88.

Simons F.; van der Hilst R., 2002. Age-dependent seismic thickness and mechanical strength of the Australian lithosphere. GRL, 29(11), 24.

Simons F.; van der Hilst R., 2003. Seismic and mechanical anisotropy and the past and present deformation of the Australian lithosphere. EPSL, 211, 271-286.

Simons F.; van der Hilst R.; Montagner J.P.; Zielhuis A., 2002. Multimode rayleigh wave inversion for heterogeneity and azimuthal anisotropy of the australian upper mantle. Geophysical Journal International, 151, 738-754.

Simons F.; van der Hilst R.; Zuber M., 2003. Spatiospectral localization of isostatic coherence anisotropy in Australia and its relation to seismic anisotropy: Implications for lithospheric deformation. Journal of Geophysical Research, 108(B5), doi:10.1029/2001JB000704 ETG 8.

Simons F.; Zielhuis A.; van der Hilst R., 1999. The deep structure of the Australian continent from surface wave tomography. Lithos, 48, 17-43.

Simpson F., 2001. Resistance to mantle flow inferred from the electromagnetic strike of the australian upper mantle. Nature, 412, 632-635.

Simpson F., 2002. Intensity and direction of latice-preferred orientation of olivine: Are electrical and seismic anisotropies of the Australian mantle reconcilable? EPSL, 203, 535-547.

Simpson F.; Bahr K., 2005. Practical Magnetotellurics. Cambridge University Press. 270 .

Simpson F.; Tommasi A., 2005. Hydrogen diffusivity and electrical anisotropy of a peridotite mantle. Geophysical Journal International, 160(3), 1092-1102.

Siripunvaraporn W.; Egbert G., 2000. An efficient data-subspace inversion method for 2-d magnetotelluric data. Geophysics, 65(3), 791-803. 
Siripunvaraporn W.; Egbert G.; Lenbury Y., 2002. Numerical accuracy of magnetotelluric modeling: A comparision of finite difference approximations. Earth Planets Space, 54, 721-725.

Siripunvaraporn W.; Egbert G.; Lenbury Y.; Uyeshima M., 2005a. Threedimensional magnetotelluric inversion: Data-space method. PEPI, 150, 3-14.

Siripunvaraporn W.; Egbert G.; Uyeshima M., 2005b. Interpretation of twodimensional magnetotelluric profile data with three-dimensional inversion: Synthetic examples. GJI, 160, 804-814.

Smith J.T., 1995. Understanding telluric distortion matrices. Geophysical Journal International, 122, 219-226.

Smith J.T., 1997. Estimating galvanic-distortion magnetic fields in magnetotellurics. Geophysical Journal International, 130, 65-72.

Soyer W., 1998. Elektromagnetische Arraymessungen im westlichen Rheinischen Schiefergebirge: Auswertung langer Perioden mit dem Z:H-Verfahren. Master's thesis, Universität Göttingen.

Soyer W., 2002. Analysis of geomagnetic variations in the central and southern Andes. Ph.D. thesis, Freie Universität Berlin.

Soyer W.; Brasse H., 2001. A magneto-variation array study in the central Andes of N Chile and SW Bolivia. Geophysical Research Letters, 28, 3023-3026.

Spence A.; Finlayson D., 1983. The resistivity structure of the crust and upper mantle in the central Eromanga basin, Queensland, using magnetotelluric techniques. Journal of the Geological Society of Australia, 30, 1-16.

Steveling E., 2000. Rap operating instructions for long period MT dataloggers. Technical report, University of Göttingen. V. 4.08.

Steveling E.; Leven M., 1992. Ein Datenlogger für niederfrequente geophysikalische Messungen. 14. Kolloquium zur elektromagnetischen Tiefenforschung in Borkheide vom 25-29.5.1992. Deutsche Geophysikalische Gesellschaft.

Swift C., 1967. A magnetotelluric investigation of an electrical conductivity anomaly in the south western United States. Ph.D. thesis, Cambridge, MA.

Unsworth M.; Bedrosian P., 2004. Electrical resistivity structure at the SAFOD site from magnetotelluric exploration. Geophysical Research Letters, 31, doi:10.1029/2003GL019405.

Utada H.; Munekane H., 2000. On galvanic distortion of regional three-dimensional magnetotelluric impedances. Geophysical Journal International, 140, 385-398. 
Veevers J., editor, 1984. Phanerozoic Earth History of the Australian continent. Clarendon Press, Oxford.

Wade B.; Barovich K.; Hand M.; Scrimgeour I.; Close D., 2006. Evidence for early Mesoproterozoic arc magmatism in the Musgrave Block, Central Australia: Implications for Proterozoic crustal growth and tectonic reconstructions of Australia. Journal of Geology, 114(1), 43-63.

Wait, 1953. Propagation of radio waves over a stratified ground. Geophysics, 19, 416-422.

Wang L.; Lilley F., 1999. Inversion of magnetometer array data by thin-sheet modeling. Geophysical Journal International, 137, 128-138.

Weaver J.; Agarwal A.; Lilley F., 2000. Characterization of the magnetotelluric tensor in terms of its invariants. Geophysical Journal International, 141, 321-336.

Weckmann U.; Ritter O.; Haak V., 2003. A magnetotelluric study of the Damara Belt in Namibia 2. MT phases over 90 degree reveal the internal structure of the waterbergrg fault/omaruru lineament. Physics of the Earth and Planetary Interiors, 138, 91-112.

Weidelt P., 1972. The inverse problem of geomagnetic induction. Zeitschrift für Geophysik, 38, 257-289.

Wessel P.; Smith W., 1998. New, improved version of the generic mapping tools released. EOS Trans., 79, 579, http://gmt.soest.hawaii.edu/.

Wiese H., 1962. Geomagnetische Tiefentellurik Teil II: Die Streichrichtung der untergrundstrukturen des elektrischen Widerstandes, erschlossen aus geomagnetischen Variationen. Pure and Applied Geophysics, 52(1).

Wingate M.; Pisarevsky S.; Evans D., 2002. Rodinia connections between Australia and Laurentia: no SWEAT, no AUSWUS. Terra Nova, 14(2), 121-128.

Woods D.; Lilley F., 1979. Geomagnetic induction in central Australia. Journal of Geomagnetism and Geoelectricity, 31, 449-458.

Woods D.; Lilley F., 1980. Anomalous geomagnetic variations and the concentration of telluric currents in south-west Queensland, Australia. Geophysical Journal of the Royal astronomical Society, 62, 675-689.

Yee E.; Paulson K., 1988. Concerning dispersion relations for the magnetotelluric impedance tensor. Geophysical Journal, 95, 549-559.

Yee E.; Paulson K.V., 1990. Reply to the 'comments on: Concerning dispersion relations for the magnetotelluric impedance tensor' by G. D. Egbert. Geophysical Journal International, 102, 9-13. 
Yin C.; Maurer H.M., 2001. Electromagnetic induction in a layered earth with arbitrary anisotropy. Geophysics, 66(5), 1405-1416.

Zhang P.; Roberts R.; Pedersen L., 1987. Magnetotelluric strike rules. Geophysics, 52(3), 267-278. 


\section{Acknowledgments}

There are many people that supported me in different stages of this project. Most prominent are my supervisors, Prof. Karsten Bahr and Dr. Fiona Simpson. They developed the Australian field campaign and promoted the development of this thesis. Thanks to Dr. Graham Heinson I was invited to the University of Adelaide, South Australia as guest scientist. During the seven month I spend in Australia I could finalize the field work organization and get in contact with many scientists involved in geoscientific research in Australia. Prof. F.M.E. "Ted" Lilley invited me to give a talk at the Australian National University, Canberra and offered his view of the conductivity structures in central Australia. Thanks to Prof. Andreas Tilgner for agreeing to be the second examiner of this thesis.

Several people at the University of Göttingen helped with aspects of this work. Prof. Ulrich Schmucker gave many helpful comments and spend hours of discussions about electromagnetic depth sounding theory, in particular regarding the GDS to MT conversion algorithm. Dr. Arno Ickler was the one to ask regarding problems with data processing and Fourier transform theory. Special thanks to Max Moorkamp for many discussions and for help with the fieldwork in central Australia. Manfred Herden was at hand to help with the shipping and Dr. Martin Leven and Wilfried Steinhoff with the maintenance of the field equipment.

Thanks to Alexander Gatzemeier, Max Moorkamp, Katherine Selway and Gunnar Hanekop for helpful comments on earlier versions of this manuscript.

I thank the rest of the staff and students of the Geophysical Institute, University of Göttingen for a good time, in particular the members of the electromagnetic depth sounding group, Dennis Rippe, Arne Lüllmann, Erik Wiebalck, Benjamin Bochmann, Mark Blome, Alexander Gatzemeier and Max Moorkamp.

Thanks to Nadine for support in the last years.

This project was funded by a Lichtenberg scholarship of the Göttingen Graduate School of Physics. The field campaign in central Australia was financed by the Geophysics Institute of the University of Göttingen. 
Acknowledgments 


\section{Lebenslauf}

\section{Persönliche Daten}

Name:

Ole Hanekop

Geburtsdatum:

21.01.1978

Geburtsort:

Göttingen

Staatsangehörigkeit: Deutsch

Familienstand:

In Partnerschaft, 1 Kind (Maja Hanekop, *23.05.2006)

\section{Schulbildung}

1984-1988

1988-1990

1990-1994

1994-1997

\section{Zivildienst}

09/1997-10/1998
Grundschule, Brüder Grimm Schule Göttingen

Orientierungsstufe an der KGS Göttingen

Sekundarstufe I auf dem Gymnasialzweig der KGS Göttingen Sekundarstufe II an der Geschwister Scholl Gesamtschule (KGS) Göttingen, Abschluß: Abitur

Zivildienst an der Christopherusschule Göttingen

(Betreuung geistig behinderter Jugendlicher)

\section{Studium}

$10 / 1998-10 / 2000$

Grundstudium Physik (Diplom) an der Georg-AugustUniversität Göttingen

$10 / 2005$ Vordiplom Physik

$10 / 2000-5 / 2003$

Hauptstudium Physik an der Georg-August-Universität Göttingen

$2 / 2002-6 / 2002$

Auslandssemester an der Victoria University Wellington, Neuseeland

5/2002-5/2003 Diplomarbeit am Institut für Geophysik, Georg-AugustUniversität Göttingen bei Prof. K. Bahr. Thema der Arbeit: "Zeitliche und räumliche Verzerrung von elektrischen Feldern in seismotektonisch aktiven Gebieten"

$5 / 2003$ Diplomprüfung Physik

seit $7 / 2003$ Graduate School of Physics am Institut für Geophysik der Georg-August-Universität Göttingen. Thema: "Large scale resistivity surveys combining magnetic and magnetotelluric observations -Examples from central Australia-" 